\title{
RESEARCH IN PARTICLE PHYSICS
}

\author{
Progress Report \\ for funding period $6 / 1 / 92-1 / 31 / 93$
}

J. Scotl Whitaker, Program Director

Boston University

Department of Physics

Boston, MA 02215

September, 1992

\section{DISCLAIMER}

\begin{abstract}
This report was prepared as an account of work sponsored by an agency of the Unired States Government. Neither the Uniced States Goyernment nor any agency thereof, ror any of their employees, makes any warranty, express or implied, or assumes any legal liability or responsibility for the accuracy, completeness, or usefulness of any information, apparatus, product, or process disclosed, or reptesents that its use would not infringe privately owned rights. Reference herein to any specific commercial product, process, or service by trade name, trademark, manufacturep, or otherwise dos not necessarily constitute or imply its endorsement, recommendation, or favoring by the United States (jovernment of any agency thereof. The views and opinions of authors expressed herein do nor necessarily state or reflect those of the United States Government. or any agency theroof.
\end{abstract}

\section{Prepared for}

THE U.S. DEPARTMENT OF ENERGY AGREEMENT NO. DE-FG02-91ER40676 


\section{TABLE OF CONTENTS}

Introduction and Description of Facilities ....................... $i$

Task A: Colliding Bearns Project $\ldots \ldots \ldots \ldots \ldots \ldots \ldots \ldots \ldots \ldots \ldots \ldots, 1$

Task B: Accelerator Design Physics ........................... 2-1

Task C: Particle Astrophysics Group (MACrO) $\ldots \ldots \ldots \ldots \ldots \ldots \ldots \ldots . . \ldots \ldots$

Task D: Neutrino Astrophysics and Proton Decay ................. 4-1

Task E: Theoretical Particle Physics $\ldots \ldots \ldots \ldots \ldots \ldots \ldots \ldots \ldots \ldots \ldots \ldots$ 5. ]

Task F: Muon g-2 Experiment at BNL ...................... 6-1

Task J: Topics in Particle Physics.......................... 7.1

Task K: SSCintCal Project .............................. 8-1

Task L: SSC Detector Development ......................... 9-1

Task N: Topics in Elementary Particle ?hysics .................. 10 1

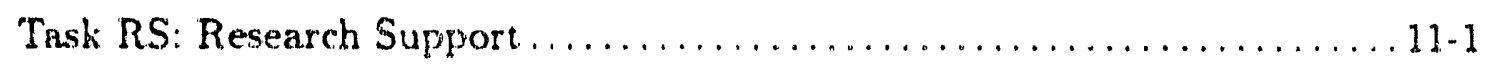




\section{INTRODUCTION}

This proposal presents the research accomplishments and ongoing actirities of Boston University researchers in high energy physics. The program was supported by contracts from the Department of Energy for six years, anc is concluding the first period of grant support. We are active in eight principal areas:

- Task A: Colliding Beams - physics of electron-positron annihilation

- Task B: Accelerator Design Physics - advanced accelerator design

- Task C: MACRO Experiment - magnetic monopoles and cosmic rays

- Task D: Proton Decay - search for nucleon instability and study of nonaccelerator physics

- Tasks E, J, and N: Particle Theory - theoretical high energy particle physics

- Task F: Muon G-2 - measurement of the anomalous magnetic moment of the muon

- Task K: SSCintcal - calorimetry for the GEM Experiment

- Task L: Muon detectors for the GEM Experiment at the SSC.

There is a great deal of interaction between the participants in these projects and considerable overlap amoiag the groups. The shared resources, facilities, and expertise available in this setting enhance the productivity of each program. Each task has designated Principal Investigators; overall management of the program is supplied by a Project Director and a Grant Adrninistrator. Administration of the program is discussed under 'Task RS. This approach to research and management improves administrative efficiency and provides coherent oversight of all aspects of the program.

The body of the proposal is devoled to detailed discussions of each of the tasks. The total budget request for the program appears in a summary chapter along with a general budget discussion and budget requests and explanations for each of the tasks.

\section{DESCRIPTION OF FACILITIES}

The HEP research program at Boston University has received excellent support from the University in every aspect. Our research facilities, described in detail below, are upto-date and are superior to those to be found in most universities. The Physics Research Building that houses our research program was built to our specifications; it includes 
mechanical and electronics shops and eight thousand-square-foot lab spaces. We have constructed a clean room for construction of particle detectors in a dust-free environment. The University has provided well over one million dollars of capital funds to furnish the labs with the tools and instruments necessary for HEP research.

The research projects benefit enormously from the availability of excellent facilities support for mechanical and electronic design and fabrication and for computing. The following sections highlight the major aspects of these facilities.

\section{Mechanical Facilities}

The Scientific Instrument Facility is located in the basement of the Physics Research Building and is administered by Physics Department personnel. This facility is a superblyequipped machine shop with a staff of eight machinists and an assembly team of three technicians. The shop has 5,000 square feet of climate controlled workspace with truck access, crane coverage, and a high bay area. The shop has a full complement of machines: vertical and horizontal mills, high-capacity lathes, high-precision lathes, a boring mill, and a grinder. The shop also has all the machinery needed for stock preparation, including a large shear, an automatic cutoff saw, and a large bending brake. In addition, the shop has complete welding and leak-checking capabilities. A shop is only as good as the people who work there, and we are fortunate to have an outsianding team of skilled, experienced insirument makers. The shop director, George Pisiello, has over 30 years experience in scientific instrument design and fabrication; he is known throughout the nation for his expertise, particularly in high vacuum systems.

The other outstanding feature of the shop is the Computer Numerically Controlled equipunent:

- Olkuma MV3 machining center

- Okuma MV5 machining center

- Okuma Lathe

- Tsugani Swiss Lathe.

These high precision, high capacity machines give our facility a production capability both for quality and quantity - that is perhaps unequaled at any other university. All these CNC machines are connecied by DNC's to our CAD/CAE systern, Anvil 5000 software running on a Sun $4 / 470$ platform. The SIF staff are equipped with workstations at which 
the CNC programs are prepared and then downloaded to the machines on the shop floor. A custom post-processor facilitates the generation of machine codes. Of the staff of eight, two are fully occupied with the CNC side of the facility, and two more of the machinists have the ability to fill in when needed.

With this facility, we have been able to make major contributions to the fabrication of HEP experiments. We fabricated many components for the SLD experiment, including pieces for the drift chamber, the Cherenkov ring-imaging detectors, and the liquid argon calorimeter. Presently, a large fraction of the facility's capacity is devoted to work for the new muon g. 2 ring being built at Brookhaven National Laboratory.

\section{Electronics Facilities}

The Electronics Design Facility is located on the fourth floor of the Physics Research Building. It has a staff of three engineers and three techniciaris, and regularly employs students as additional workers are required. The EDF offers advanced electronics design, prototyping, and lesting capability in support of our research program. A major design tool is the electronics CAD package from Mentor, running on Apollo workstations. With this resource, circuits are not only designed but can also be simulated before any actual prototyping is done. Advanced analog and digital circuits have been designed and produced. Some examples include: for CP/LEAR a 64-channel TDC system with 100 psec resolution and digitization and readout of all channels in one microsecond; a 200 Megasample/sec waveform digilizer system for the MACRO experiment; and, currently in development, a GaAs ASIC digital TDC with continuous readout for the DUMAND experiment. The EDF has high-speed test equipment and operates a data acquisition system to be able to test experimental equipment. In-house fabrication capacity is limited; production is better handled in industry and the EDF staff are experienced in managing work contracted out to industrial vendors.

\section{Computing facilities}

The computing environment in which we work is diverse and comprehensive, with many machines and many software resources. The Physics Department has two full-time computing staff who maintain the systems and the software. Our computing facilities include the following machines: 
- Vax 8600 , plus four microvaxes and vaxstations

- Encore Multimax

- Sun 4/470 server, and a dozen or so smaller Sun machines

- Connection Machine CM2, with Sun 4/470, Sun 4/280, and Vax 6400 front ends

- Eight DECstations

- Four Silicon Graphies workstations.

- SGI 4D/340S and five SGI Indigo work stations.

Our computational facilities are linked together by broad-band networks so that every office has a connection to every machine; we are linked by all the major networks to the rest of the world as well. 
TASK A: COLLIDING BEAMS PROJECT

Faculty: $\quad$ Professor Scott Whitaker

Research Assistant Professor Anthony S. Johnson

Research Assistant Professor Jarnes T. Shank

Graduate Student: John Coller

\section{Introduction}

The Colliding Beams Group currently has research activities in two major areas: in the SLD project at SLAC and in the GEM Experiment at the SSC. To complement this program we are developing a role in the Beijing Spectrometer (BES) experiment at the Beijing Electron Positron Collider; we will participate in the data analysis and contribute to the hardware upgrades now being planned. The staffing of the $C B$ Group is changing; Assistant Professor Bob Wilson is departing to take a faculty position z.t Colorado State University, where he will continue his collaboration in the SLD and the BES efforts.

Within the SLD, our primary activity has been on the endcap CRID. As discussed below, we have constructed, installed, and are in the process of commissioning the proportional wire detectors for the endcap ring-imaging Cherenkov counters. We are also a major contributor to the on-line and off-line software for the exputiment. During the next several years we will collect and analyze data on the production and decays of $Z^{\circ}$ 's. The BES analysis effort will be centered at SLAC. Some of the software develuped for the SLD is being adapted for BES use. The buildup of analysis on SLD and BES data will dovetail nicely with the conclusion of our construction effort in the SLD.

In the past several years we have pursued a serjous involvement in the physics program of the Superconducting Super Collider. For the past year we have been working in the GEM collaboration, where we are one of the principal groups working on the endcap muon system. The combination of detector development and construction for the SSC and analysis of data from SLD and BES will provide a balanced physics program for this group. The SSC-related work is presented under Task L; Task A covers ihe electronpositron research activities of the group. 


\section{SLD Activities}

The SLD has been running for the past year; the detector is operating well; the hardware and software systems are shaken down. Luminosity performance has improved dramatically: we are now logging approximately $1,000 Z^{\circ}$ 's per week. The polarization of the electron beam is an important part of the SLC capability. It will make the SLD competitive with the LEP experiments in some physics topics despite the luminosity advantage of the CERN machine. The polarization of the electron beam at the interaction point is typically $22 \%$, and there are prospects for improved photocathodes which would dramatically increase the polarization. More than 8,000 "polarized" $Z^{\circ}$ 's have been logged, and there is the possibility for that amount again before the end of the present run.

Analysis of SLD data is well under way. Preliminary results for the left-right asym. metry $A_{L K}$ and for QCD studies were presented at the ICHEP conference in Dallas. For many of the detailed studies of the standard model, systematic errors dominate the experimental measurement. In these cases, the SLD will again be competitive with the LEP experiments.

The forward region of the SLD will be particularly important in the measurement of asymmetries. These measurements are a sensitive probe of the Standard Model and may give evidence for new particles beyond the kinematic range of direct production experiments. Coverage of the region between 20 and 40 degrees in polar angle doubles our asymmetry measurement capabilities. The endcap CRID (Cerenkov Ring Imaging Device) project is a central part of this program, and its timely completion will increase the capability of the SLD to exploit the competitive advantage that polarization gives us. The Boston University group has been central to achieving that gand.

\section{CRID Endcap Detectors}

The endcap CRID is a ring-imaging gas Cherenkov detector that will provide particle identification over a wide momentum range. Particles traversing the endcaps emit Cherenkov radiation in the freon radiator; the Cherenkov photons are focused by mirrors onto a quartz-walled drift box filled with a TMAE-laden gas, in which the photons interact to create single photoelectrons. The electrons drift to a picket fence proportional detector with 7 micron diameter carbon "wires" on a .138 inch pitch. Drift time measurement, wire location, and charge division along the wires permit reconstruction of the photon interaction point with millimeter precision in each coordinate. 
Our previous report detailed the design, construction, and test of these detectors. The production appeas to have been very successful. All of the devices passed the major QC/QA tests at the University of Cincinnati and were integrated into drift box assemblies being constructed there. Of the 1,280 anodes in the 10 chambers, only 4 have problems.

Five of the detectors have been installed in the North endcap of the SLD. Installation of high voltage, cooling water, heating circuits, gas supply, and readout electronics has been completed. Commissioning of these detectors is in progress. Hookup to the final ethane drift gas system awaits approval by the safety committee and concurrence from other systems that will be affected. An independent freon radiator gas system is being developed for the endcaps so they can be operated independently of the barrel CRID system. Operation of the North endcap CRID could be possible before the end of the present run.

On the South endcap, all the detectors and drift boxes are installed. Readout electronics packages are completed for four of the five detectors; the fifth will be done soon. Installation of the electronics and hookup of the utilities will require opening the SLD doors; this may not be possible until after the and of the current run, sometime this Fall.

Particle identification in the endcaps is achieved by combining velocity measurements made by the CRID with momentum measurements from the ECDC. Considerable improvement in the performance of the ECDC has recently been achieved by tuning data acquisition parameters. Prospects are now encouraging for successful operation of the combined endcap systems.

Sofiware

Over the past five years we bave been heavily involved with the development of the online and offline software for the SLD experiment. In particular, Johnson $1 \cdots$ a been a key member of the software team, designing and developing several major areas of the SLD off-line reconstruction and analysis code and supervising the development of all SLD soft ware utilities.

SLD has emphasized the use of interactive data analysis tools to aid in the rapid analysis of data once it is obtained and to help in the development and debugging of Monte-Carlo and reconstruction programs. This has entailed the development of a number of innovative analysis tools, including:

- Advancement of the MORTRAN macro preprocessor for FORTRAN. 
- Development of a data management system, JAZELLE.

- Development of the Distributed Update Control System DUCS.

- Development of a graphical user interface, the Motif Interactive Data Analysis Shell MIDAS.

These powerful utilities form the core of the SLD analysis system. They are being adapted now for other applications, including possible use in the BES and GEM Experiments as will be discussed below.

\section{Planned SLD Activities}

As discussed above, there remains a considerable amount of work to finish commissioning of the endcap CRID system. The schedule for this depends on some factors largely beyond our control, such as door openings that are needed to provide access and to allow interruption of systems that are shared with the barrel 'ORID. Bringing the endcap system into operation will be our principal effort on SLD until the completion of that task. Wilson, from his new Colorado State University position, will continue to be heavily involved in this work.

With present conditions, the prospects are that the current run will yield well in excess of $10,000 Z^{\circ}$ 's. Members of our group are well-prepared to take advantage of this data. The amount of data with the endcap CRID operating will be small, however. The SLD collaboration and the SLAC management have plans to run in 1993, and this will provide the primary data sample for the endcap analysis. Discussion has been opened concerning a 1994 run; this will be justified on the basis of the physics performance and the competitiveness of the SLD and the SLC.

\section{The Beijing Spectrometer (BES)}

It has been clear that the SLD will terminate data-taking after a few years, and our group has spent part of the past year planning for our next project. Our contributions to the SSC are successful and we plan to pursue them. To keep a balanced program during the years until the SSC operates we want to be involved in a project which will provide world class physics for at least the next five years. We have considered a number of options; our preferred choice is to pursue the opportunities in $e^{+} e^{-}$physics at the Beijing Electron Positron Collider (BEPC). 
$\mathrm{BEPC}$ is a $2.0-2.8 \mathrm{GeV} /$ beam $\mathrm{e}^{+} \mathrm{e}^{-}$collider. It was designed with substantial assistance from accelerator physicists at SLAC and was based upon the very successful SPEAR storage ring. Experiments at SPEAR provided an impressive range of physics results for over a decade. The Beijing collider will be the source for the next generation of experiments in the very fruitful energy region around the $\mathrm{J} / \psi, \tau$, and charm thresholds. It is expected to achieve a maximum value of $1.7 \times 10^{31} \mathrm{~cm}^{-2} \mathrm{sec}^{-1}$ at an energy of $2.8 \mathrm{GeV}$ per beam. It will have a luminosity 4 to 5 times that of SPEAR, and it will be scheduled for dedicated High Energy Physics runs 2 to 3 times as much each year as SPEAR was able to manage.

A collaboration of Chinese physicists was formed to build a detector, the Beijing Spectrometer (BES), which was nearly a direct copy of the Mark III detector at SPEAR. After several years of running, it became clear that collaboration with U.S. physicists could provide needed expertise in analysis. A group of U.S. institutions have joined the project, with Walter Toki of CSU as spokesman. We will participate in operating the experiment and analyzing the data, and we also propose to take part in the upgrades needed to maintain and improve the performance of the detector.

As discussed in our previous report, there is a wealth of physics to be studied at BEPC. The enerigy region around 3-5.6 GeV center of mass has been studied in $\mathrm{e}^{+} \mathrm{e}^{-}$colliders for many years by experiments such as Mark I, DASP, Crystal Ball, CLEO, Mark III etc. None the less, there are important physics topics remaining to be resolved.

One of the first important projects at BEPC has been to do a careful scan across the $\tau$ threshold to determine the $\tau$ mass more precisely. This measurement is important due to the dependence of the decay width on the mass:

$$
\Gamma(r \rightarrow \mu \nu \bar{\nu})=G_{r}^{5} m_{r}^{3} / 192 \pi^{3}
$$

A similar expression exists for $\mu$ decay. By using the measured $\tau$ lifetime and branching ratio one can test whether the weak coupling constants $G_{\tau}$ and $G_{\mu}$ are equal. With current values there is a $2 \sigma$ difference. The suspicion has been that the $m_{r}$ is incorrect. The result from the BES scan, as presented by F. Porter at ICHEP 92 (Dallas), is $\mathrm{m}_{r}=$ $1776.9 \pm 0.5 \pm 0.3 \mathrm{MeV}, 7.2 \mathrm{MeV}$ lower and considerably more precise than the world average of $1784.1(+2.7,-3.6) \mathrm{MeV}$. Argus and CLEO also presented new tau mass determinations, in agreement with the BES result but with significantly larger errors. The new mass value improves the agreement with Standard Model predictions considerably. 


\section{Planned Activities}

We are becoming active in several areas of BES software, including code maintenance on the VAX and porting BES codes to UNIX platforms, where the data throughput could be greatly increased due to the availability of powerful workstations and computing resources. We are exploring possible applications at BES of the DUCS and MIDAS software utilities developed for the SLD. We expect to become involved in the physics analysis very soon; Coller will do his thesis research on this project, the analysis here complementing his hardware experience from the SLD and SSC work.

The electronic and mechanical shop facilities at Boston University will enable us to make major contributions to the hardware upgrades to BES. One of the major tasks will be to refurbish or replace the main drift chamber (MDC) of BES. The MDC is demonstrating some of the classic symptoms of drift chamber ageing, and we are studying the relative advantages of restringing or replacing the chamber. Replacment would have the major advantage of limiting the down time of the experiment, and at present this is the more likely route. The MDC has two-meter diameter endplates wich precisely located holes for positioning the wires. Machining the end plates for a replacement drift chamber would be particularly well suited to our facilities at Boston University. We are presently working to acquire a large CNC horizontal milling machine that would have the capacity to do thin work. This will be a unique capacity among Universities in the U.S. or in China.

We are also doing some preliminary work in the electronics developments required to improve the performance of the BES shower counters. These gas detector-based calorimeters are currently operated in streamer mode. Changing to operating in proportional mode would improve resolution and linearity but would require modification of the data acquisition system with increased front end gain. We are studying the possibility of making a hybrid low-gain (x10) amplifier that could be inserted into the present sample-and-hoid modules to accomplish this.

A proposal for the upgrade program and the role of the U.S. participants will be presented to the D.O.E. this Fall. The spirit in which we at Boston University are undertaking these upgrade tasks is to identify areas where we can make a unique contribution to the project through our superior infrastructure and facilities. 


\section{Budget Discussion}

The budget page for the $C B$ group presents the base funding request for the year starting February 1, 1993. The bottom line is scaled from the current funding levels, with an increase in foreign travel required for BES. We have moved into this task's buctget the half of the salary for J. Shank that previously was derived from SSC funds under Task L. This adjustment has removed our dependency on SSC funds for base program salary support. Once the status and schedule for BES upgrades is settled, equipment funds will be requested in accord with our upgrade responsibilities. 


\section{Recent Publications by Members of the CB Group}

1. DUCS: A Fully Automated Code and Documentation Distribution System. A. S. Johnson (Boston U.), B. Saitta (Ferrara U.), O. Gervasi (Perugia U.), G. Bower, A. Rothenberg (SLAC), A. Waite (Victoria U.), SLAC-PUB-5230, Apr. 1990. Presented at Computing in High Energy Physics Conf., Santa Fe, NM, Apr. 9-13, 1900.

2. JA.ZELLE: An Enhanced Data Management System for High-energy Physics. A.S. Johnson (Boston), Martin Breidenbach, H. Hissen, Paul F. Kunz, D. Sherden (SLAC), T. Burnett (Washington U., Seattle), SLAC-PUB.5231, Apr. 1990. Presented at 8th Computing in High Energy Phyzics Conf., Santa Fe, NM, Apr. 9-13, 1990.

3. Electrostatic Design of the Barrel CRID and Associated Measurements. CRID Group (K. Abe, et al.), SLAC-PUB-5214, Apr. 1990. Contributed to Ini. Conf. on Instrumentation for Colliding Beam Physics, Novosibirsk, USSR, Mar. 15-21, 1990.

4. Construction and Testing of the SLD Cerepkov Ring Imaging Detector. M. CavalliSforza et al. SLAC-PUB-5123, Jan. 1990. Presented at IEEE Nuclear Science Symposium, San Francisco, CA, Jan. 15-19, 1990. Published in IEEE Trans. Nucl. Sci. 3i:1132-1141, 1990.

5. Progress in the Development of a Tracking Transition Radiation Detector. J. S. Whitaker et al, SSC Detector R\&D Symposium, Fort Worth, Texas; October 1990.

G. Test Bearn Performance of a Tracking TRD Prototype. J.T. Shank, J.S. Whitaker, et. al., CERN-PPE-91-49, Mar 1991. 13pp. Submitted to Nucl.Instrum.Methods

7. An Expression of Interest to Construct a Major SSC Detector. B. Barish, W. Willis ct. al, SSC EOI-0020, July 8, 1991.

8. Measurersent of the Mass of the Tau Lepton. J. Z. Bai et. a., SLAC-PUB-5870 and IHEP-EP-92-01, July 1992. Presented at the International Conference on High Energy Physics, Dallas, August 1992. 


\title{
'TASK B: ACCELERATOR DESIGN PHYSICS
}

\author{
Faculty: $\quad$ F. Krienen, P.I., Professor of Applied Physics \\ and Engineering \\ Graduate Students: Zhifeng Liu \\ Tournsend Zwart
}

\section{Project Summary:}

New challenges in high energy physics place increased demunds on accelerator physicists. Advanced light sources, heavy ion arcelcrators, ard the SSC require the refinement of existing accelerator technologies and the development of new ones. Boston University has assembled resources in the Department of Physics to address several interconnected questions of accelerator design, as well as to train graduate students in this field.

Several specific design issues of general nature have arisen from an experiment being pursued by other faculty in Physics to measure the muon anomalous magnetic moment or $(g-2)$ inlue. Ti: Accelerator Design Group at Boston University is working with physicists and engineers from the $(g-2)$ Collaboration in Japar, at Yale, and at Brookhaven National Laboratory to solve problems presented by the design of a precision superconducting storage ring, of both active and passive automated magnet shimming systems, and of a superconducting beam injection system. We are also engaged in the design and construction of compact electrostatic quadrupole focusing elements to be mounted inside a new storage ring vacuum chamber design. One graduate student completed his doctoral research in accelerator design physics last year. Two graduate students currently engaged in research would be supported by this task.

We envision several major tasks toward which our efforts will be directed during the coming year: continued work on the superconducting beam inflector; further development of the electrostatic quadrupoles; involvement in the "electrostatic muon kicker;" and use of computing facilities at Boston University, including the Connection Machine, for threedimensional magnetic field analysis, development of software for magnet shimming, and associated research. 
We are starting the design of the full-aperture kicker to be used for muon injection. A number generic issues must be addressed if such a device is to be realized. The "kich" must rise fairly quickly but must decay as completely as possible in $150 \mathrm{~ns}$, which is the

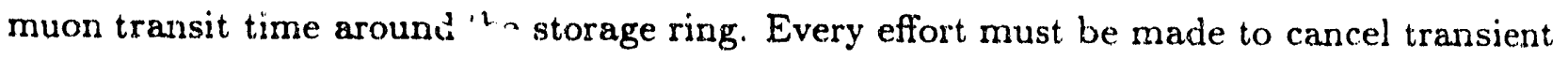
affects so that the residual fields seen by the muon bunch are of minimal impact on the $(g-2)$ precession.

The work on the superconducting beam inflector has achieved some official recognition: the patent application submitted in 1988 has successfully cleared the initial processing hurdles and has been filed with the U.S. Patent Office. The truncated double cosine theta superconducting magnet concept has been published, ${ }^{1}$ and further developments of truncation are documented in reference [2]. Recent developments concerning the electrostatic quadrupoles are a modification of final vacuum charnber design to include the entire path of the decay electron. ${ }^{3}$ The testing of the quadrupole is in an advanced stage. A significant reduction of difficulty in designing the puising electronics, in addition to an easy accommodation of the proposed NMR field mapping trolley, make this new vacuum chamber and quadrupole design more attractive and feasible. The concentration of electrostatic quadrupoles into a four-fold symmetry reduces possible pre-showering of the decay electrons ruen more."

This proposal outlines the efforts we envision in several general areas of accelerator design physics. Threr tasks on which work is currently under way are described in detail. Some of this effort is directed toward problems presented by the muon storage ring; all of it is of generic importance to accelerator design physics.

\section{Ongoing Accelerator Design Tasks}

\section{Superonducting Beam Inflector}

A superconducting inflector will be used for the rnuon $(g-2)$ experiment. We believe that the fringe fields are acceptably small. The advantage of a $\mathrm{JC}$ inflector is that the fringe ficlds will be steady-state, so that they can be shimmed out. The inflector in the muon $(g-2)$ experiment will produce a 1.47 Tesla magnetic field over a 1.8-meter length to cancel the field of the magnetic storage ring. The channel has a useful section $4.5 \mathrm{~cm}$ high and $1.8 \mathrm{~cm}$ wide. Uniformity is good to a few percent. 
We are doing intonsive research and have arrived at magnetic configurations which we believe can be made into a feasible device. Further research and development is necessary.

Using Professor Frank Krienen's innovative analytical technique, we developed highspeed computer algorithms for calculating the magnetic fields and optimizing the magnetic configuration. We have already proved their use. Figure la illustrates the magnetic configuration using these algorithms. The configuration assumes infinite length for initial calculations, and as such, the results are satisfactory to the required precision. One aspect of this problem, and an element crucial to the feasibility of the device, is the calculation of the forces and torques on the inflector. We have already calculated the self-forces (due to the current within the conductors themselves) and found them reasonable. The residual forces and torques due to the storage ring magnetic field, however, present a more claallenging problem which will require precise numerical analysis. A three-dimensional stick model ${ }^{3}$ program has been used to calculate the force on the end loops, both in the central $1.45 \mathrm{~T}$ field and in the fringe field which decreases to about $0.9 \mathrm{~T}$ near the up-stream end. In the calculation, the superconductor is divided into many straight segments, and the B-fiold is calculated using the Biot-Savart law. Each element is small enough so that it can be approximated as being in a constant field. The E field components at each segment center, due to all the other current segments, as well as those due to the fringe field of the siorage ring, are superimposed to find the total B-field. The force on each segment is then calculated, and from this the three components of the total force on the inflector are calculated. The results of this calculation are shown in Figure 1b.

As noted above, the end effects and fringe fields for a finite-length device have been calculated and studied. To provide more options, we designed another type of inflector which differs from the truncated double cosine theta type. In this design concept, a so-called "coaxial" inflector, a constant current density" superimposes a cosine theta distribution current density on a cylindrical surface. The vector potential contains a logarithmic term which turns out to give a favorable reduction in the height of the inflector together with an increase in the useful beam area.

Wr have firalized a preliminary design and will continue working to solve the problems duc to end effects. We are also studying the cooling losses in the final device. Furthermore, stray ficld measurements will be taken with the inflector immersed in an external field provided by a test magnet at Brookhaven. 
The design has been accepted by the $(g-2)$ collaboration, ${ }^{6}$ and KEK has increased its involvement in preparation for the manufacture of the inflector. The final design and manufacture of the inflector cryostat will be done at $\mathrm{BNL}$, with $\mathrm{C}$. Pai of $\mathrm{BNL}$ coordinating the design with $A$. Yamamoto of KEK, who is constructing the inflector.

KEK has nearly completed the engineering drawings and is making a short section $(0.5$ $\mathrm{m})$, after which the final version put will be put in the workshop. Completion is targeted for the summer of 1992 . W. Meng of BNL is studying the feasibility of reducing still more the stray flux seen by the coasting muons by means of a superconducting flux exclusion shield, made of superconducting tape wrapped around one end of the inflector. 'The test would require a $\mathrm{C}$-magnet with a large gap accommodating the prototype cryostat.

BNL has nearly completed the design and drawings of the cryostat for the inflector. The vacuum in the cryostat is independent from the vacuum in the storage region.

KEK suggested a reduction in the size of the superconductor in order to ease the winding procedure. This required from our side a complete recalculation of the position of the conductors and calculation of the forces.

Extensive short-sample tests have been done by BNL on tis conductor of $2 \times 3 \mathrm{~mm}^{2}$, which can carry some $3000 \mathrm{~A}$ in samples that have been bent and twisted over distances comparable with the conductor size.

Finally, it has been decided to close both ends of the infector so that muons must twice cross a layer of conductors, each amounting to $4 \mathrm{~mm}$ of aluminum and $1 \mathrm{~mm}$ of copper equivalent. This makes the winding a lot easier, although some muon losses are incurred due to multiple scattering. Closed end loops reduce the stray field by a factor of four. so that the magnetic shimming in the storage region is made somewhat easier.

\section{Prototype Vacuum Chamber and Electrostatic Quadrupole Configuration}

The precision requirements of the muon $(g-2)$ measurement demand a magnetic field homogeneity of $1 \mathrm{ppm}$. This excludes the presence of a magnetic field gradient or quadrupole component for vertical focusing of the stored muon beam. The quadrupole is therefore provided by an electrostatic field defined by high voltage electrodes mounted inside the vacuum chamber. The electric quadrupole potential is ideally a pair of hyjerbolae with asymptotes at $45^{\circ}$ with respect to the radial and vertical directions. Therefore, in the 1978 CERN muon g- 2 experiment, truncated hyperbolic electrodes above, below, and to the sides adequately approximated an electric quadrupole field. 
The combination of high voltage, vacuum, and magnetic field gives rise to a phenomenon known from the CERN experiment as electron trapping. The high voltage attracts low energy electrons from ionization of residual gas in the vacuum, while magnetic field lines restrict the electron motion vertically. There is in fact an axial drift of the plasma around the ring along the electrode inside of electric equipotentials. This charge accurnulation can lead to breakdown which will erode the electrodes and cause the vacuum to deteriorate. The finite lifetime of the muon, however, allows the high voltage to be pulsed in short duration (after $800 \mu s$ ), which minimizes the ill effects and occurrence of electron trapping.

A straight six-foot dipole magnet, now in place at Building 919, has test quadrupoles mounted in the tank with the tank immersed in the magnetic field. The vacuum tank is eight feet in length, while the electrodes are four feet long to avoid the edge effects of the magnetic field. A window at one end of the vacuum tank has allowed optical detection of the faint bluish glow associated with tive electron trapping. High voltage pulsers, one employing spark gaps, one employing high voltage solid state switches, have been tested.

At present we are trying to concentrate the electrostatic quadrupoles to four sections of 30 degrees each, arranged in a symmetric fastion. Thus the decay electrons will see less material in which they can scatter and pre-shower, although at the cost of higher pulsed voltage on the electrodes $( \pm 30 \mathrm{kV}$ ). This will be tried out shortly.

Another development deals with the simplification of the shape of the hitherto curved hyperbolic blades. Instead of the double curvature, perhaps straight segmented upper and lower electrodes and straight curved sections on the side will give acceptable field quality. Initial calculations of the electric field produced by flat, rather than hyperbolic electrodes, are promising. S. Mane from BNL is using $\vec{E}$ fields calculated from flat electrodes in his tracking program to see if the multipoles induced by the lack of curvature cause coupling to resonarces and thus cause the loss of stored muons.

\section{New Vacuum Chamber Desion and Modified CERN Quadrupoles}

Continued discussions with the detector subgroup about the collection of decay electrons have brought to light the desirability of modifying the vacuum chamber to reduce the material through which decay electrons must pass. ${ }^{3}$ Therefore, the entire auc of the decay electron trajectory, from muon decay to detection, is to be cortained inside the vacuum chamber. This is shown in Figure 2. The decay electrons traverse the tank wall 
perpendicularly to exit the vacuum tank, thereby minimizing the amount of material and hence the probability of pre-showering before their actual detection. In the CERN vacuum chamber, traversal of the vacuum wall was very much at a grazing angle, so that for a wall thickness of $1 \mathrm{~mm}$ aluminum equivalent the average path at $1.5 / \mathrm{c}$ was $3.6 \mathrm{~mm}$, and at $2.7 / \mathrm{c}$ the average path was $10.44 \mathrm{~mm}$. In the new design, the average path is less than $3 \mathrm{~mm}$ and is moreover touching the detector, so that pre-showering is of no importance.

In this new vacuum chamber design, the inner hyperbolic electrode is no longer the super-thin wall of the tank itself. Therefore, it need not be grounded as required before and it becomes possible to reduce the magnitude of the high voltage necessary for quadrupole field definition. For the four-fold symmetry the pulsing electronics need only produce \pm 30 $\mathrm{kV}$ instead of $60 \mathrm{kV}$ with respect to the grounded hyperbola in the previous design. This being the case, grounded corner protrusions lie on ideal quadrupole potentials and can be used as the support for the envisaged field mapping trolley to operate inside of the vacuum chamber.

\section{Design of the Muon Kicker}

Inflection of muons in the storage ring requires a muon kicker located about a quarter of the ring circumference downstream from the inflector. The kick amounts to about 10 mrad. Csurlly a fast magnetic kicker serves the purpose: the total pulse length can be madr compatible with the time of revolution of the muon $(\sim 150 \mathrm{~ns})$.

However, we suspect long-living eddy currents set up in the electrodes and the vacuum tank will produce an uncertainty in the actual magnetic field seem by the coasting muons. One could envisage a double magnetic kick of opposite polarity, canceling the eddy currents to acreptable levels, but present study in that line is is not very encouraging: some 16 thyratrons of $80 \mathrm{kV}$ are needed, and the current loop is chopped up in eight sections of $50 \mathrm{~cm}$ each: a very expensive proposition.

Instead, we at Boston University are considering an electrostatic kicker, requiring only iwo thyratrons charging the electrodes via a pulse transformer to $\pm 400 \mathrm{kV}$, which are then discharged with a spark gap. The electrodes and feed point of this device can be made so that virtually no eddy current effects are biasing the data. 
We intend to start some preliminary tests using existing Marx generators to reach high voltage to study the feasibility of the feedthrough insulators and breakdown in the tank. The reference to the above may be found in $\mathrm{g}-2$ note 84, July 3, 1991 (appended). The components for this endeavor are being assembled, and testing is imminent.

\section{Computer Codes for Accelerator Design}

The Accelerator Design Group at Boston University has been using the High Energy Physics Computational Facility for solving several classes of problems that arise in accelerator design. In particular, we continue to make extensive use of the POISSON Group Codes for studying two-dimensional electromagnetic problems. Besides using them in the ongoing studies of the electrostatic quadrupole and the main storage ring magnets, we will also use the POISSON codes to arrive at the optimum shape for the electrodes of the full-aperture kicker.

These codes use the successive point over-relax..tion method ${ }^{7,8}$ to solve Poisson's equation for magnetostatic fields and Laplace's equation for electrostatic fields.

In the case of the superconducting inflector, analytic methods introduced by Professor Kirienen provided results that are both exact and much faster then POISSON. The bulk of the programming of the new algorithms has been completed, and a large amount of computer time was used to optimize the inflector design. Besides the inflector, other applications (combined function magnets, dual beam facilities, etc.) were studied using the new methods. ${ }^{2}$ These algorithms, however, are limited to two-dimensional problems, and therefore cannot be applied to the study of the inflector end-turns. For this reason a program was acquired for calculating forces and torques due to three-dimensional currents. ${ }^{5}$ Several configurations of the end-turns have been analyzed with this program, but this study will continue.

Another area of ongoing interest has been the problem of pole-piece profile and shimming of the magnetic field in the storage ring magnet. During the entire design period of the ring magnet, calculations done at BNL by Jackson and Danby were checked by independent calculations carried out at Boston under the supervision of Prof. Kirienen. (During the past six to eight months calculations were also carried out at Yale using the BNL codes.) The design of the magnet is essentially fixed. A cross section is shown in Figure 3. The final pole piece profile will be slightly altered to improve the quality of the field just outside of the storage region. 
Still another task of interest to accelerator physicists, and one that lends itself to calculational techniques developed at Boston University for $(g-2)$, is a novel method to enhance positron production in a beam with the aid of a magnetic horn. This would have applications both at storage ring colliders and, especially, for linear colliders. For this study we will make use of the CERN GEANT3 program for the simulations of showers and subsequent tracking of the positrons. First trials with this program look promising; results may be expected by the end of 1992 .

\section{Proposed Efforts in 1992-93}

The major project proposed for the next year is a study of the feasibility of a pulsed electrostatic full-aperture kicker for muon injection. At present this is a generic device, which, if certain problems can be solved, can be adapted to the $(g-2)$ experiment. The advantage is complete cancellation of the effect of eddy currents on the coasting muons. The equipment will also be cheaper to build, in comparison with the heavy-current, singleloop rnagnetic kicker.

The high precision of the muon $(g-2)$ experiment places stringent requirements on the uniformity and knowledge of the magnetic field within the storage region. The uniformity must be good to the level of $1.2 \mathrm{ppm}$, nd the field should be known to $0.1 \mathrm{ppm}$. The mapping of the ficld is done with an NMR device, which is carried around the storage ring by a trolley. A prototype trolley, modified from one invented by Enrico Fermi, has been proposied by Professor Krienen. This trolley design is still under consideration for use in the $(g-2)$ experiment. Once the specifications of the NMR electronics to be carried on board are specified, we will continue the development of the Fermi trolley.

Work will continue in the area of electrostatic quadrupole design, which approaches finalization. Smaller modular units are now being envisaged, which would ease the manufacture and possible replacement if breakdown damages locally a quadrupole element.

Finally, as an exercise in particle optics, we will study a miniature magnetic horn for the production of an enhanced positron beam. 


\section{References}

1. F. Krienen, D. Loomba, W. Meng, "The Truncated Double Cosine Theta Superconducting Magnet," Nucl. Instr. and Meth. A283, 5 (1989).

2. F. Krienen, D. Loomba, W. Meng, "Development of Non-ferrous Superconducting Magnet," g-2 Note No. 58.

3. F. Krienen and D. Brown, "New Vacuum Tank Reducing Pre-Showering of Decay Electrons," g-2 Note No. 62 (1990).

4. Y. Y. Lee (BNL), personal communication.

5. The program FLD3D was acquired from its author, R. D. Pillsbury (MIT).

6. "A New Precision Measurement of the Muon g-2 value at the level of $0.35 \mathrm{ppm}$," AGS Proposal 821, Sept. 1985, revised Sept. 1988.

7. A. M. Winslow, "Numerical Solution of the Quasilinear Poisson Equation in a Nonunjform Triangular Mesh," J. Comp. Phys. 2, 149 (1967).

8. R. F. Holsinger, User's Guide to POISSON Group Codes, LANL-NM-87545. 
Figure 1a. The flux lines in the inflector with the new design, which employs the smaller diameter superconductor.

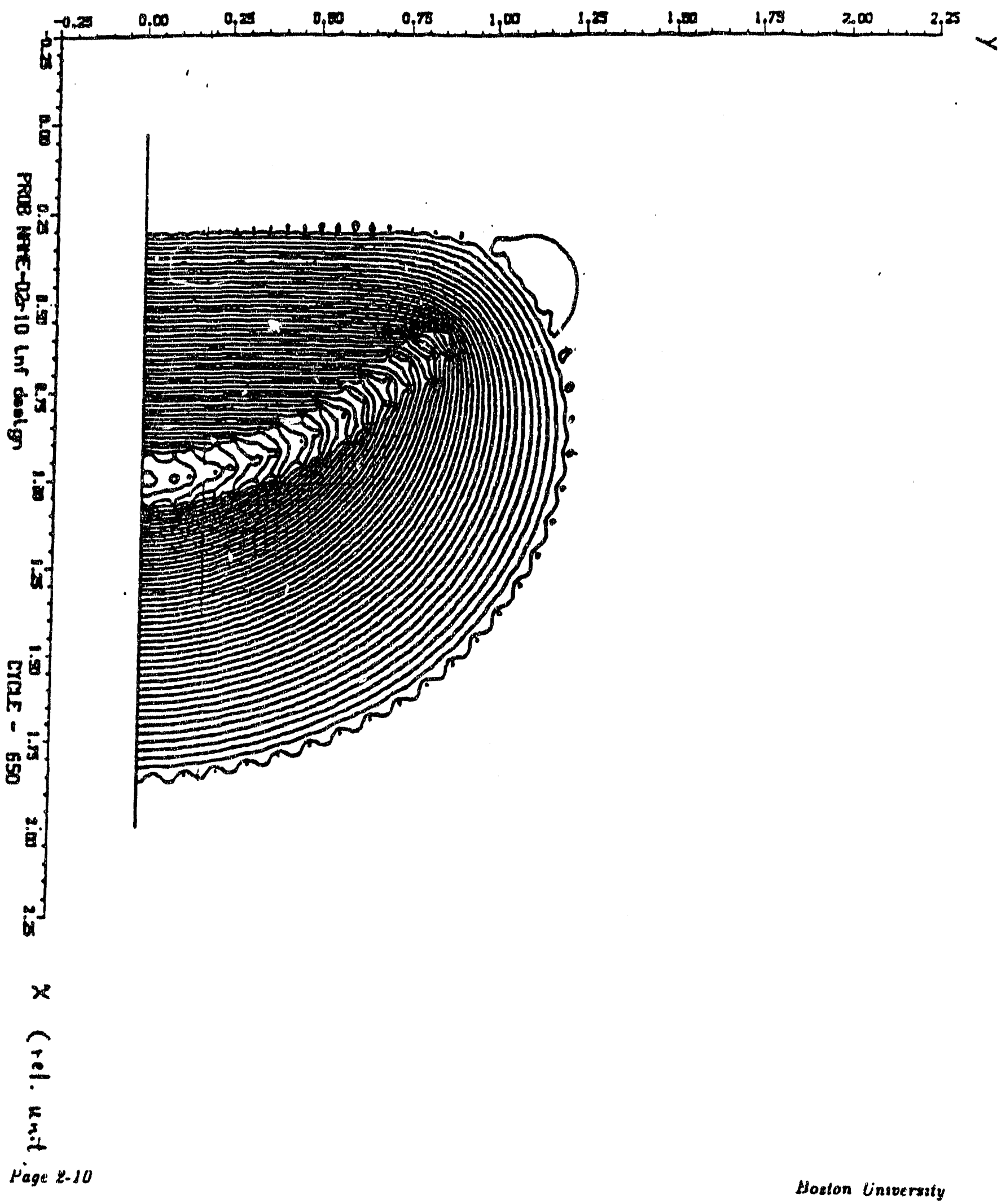




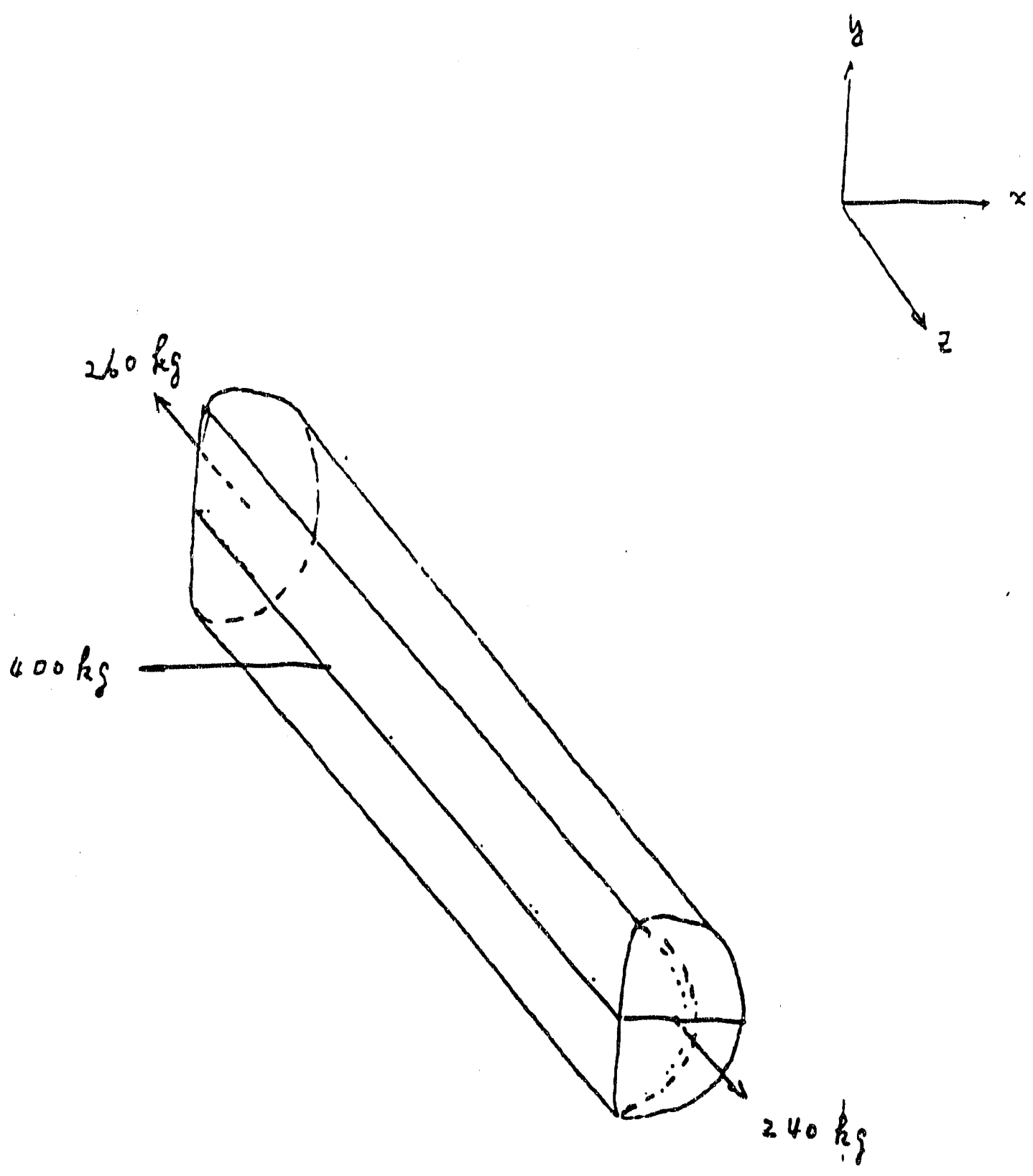

Figure Ib. The forces on the superconducting inflector as calculated from the stick model described in the text. The beam enters from the upper left in the figure. The $400 \mathrm{~kg}$ force on the side arises from the non-uniform fringe field. 


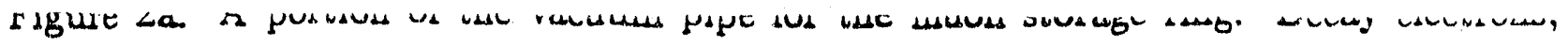
which have lower energy than the stored muons, spiral in aind exit transverse to the exit window.

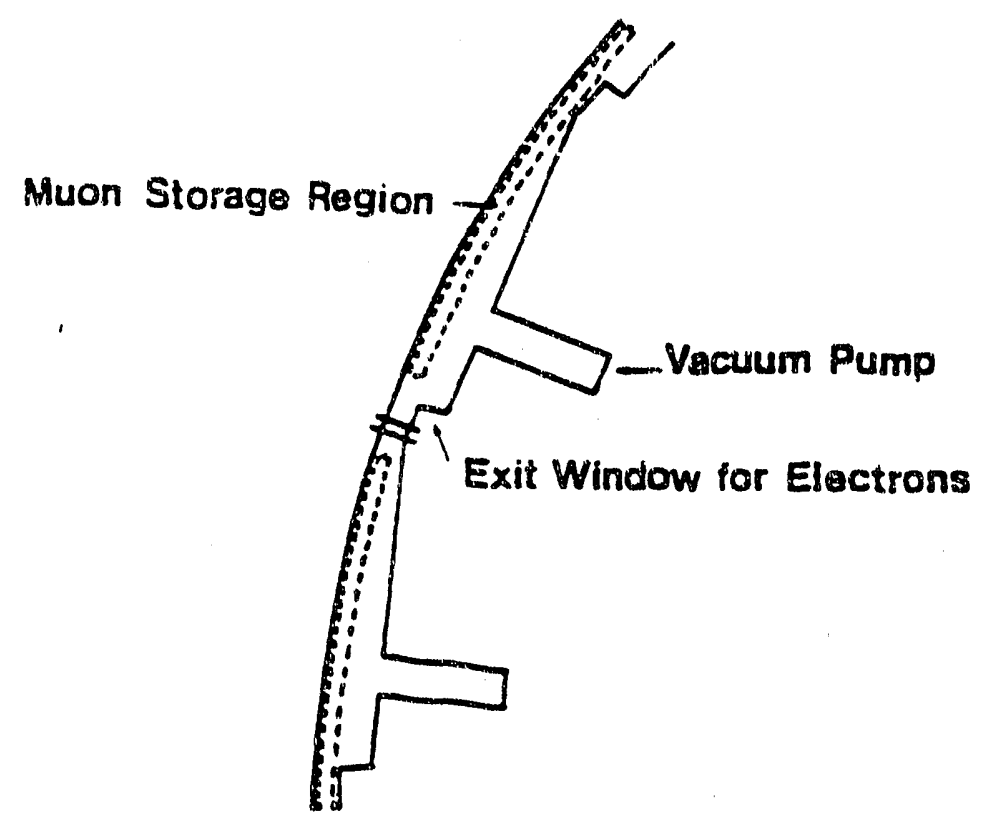

Figure 2b. View of the vacuum chamber.

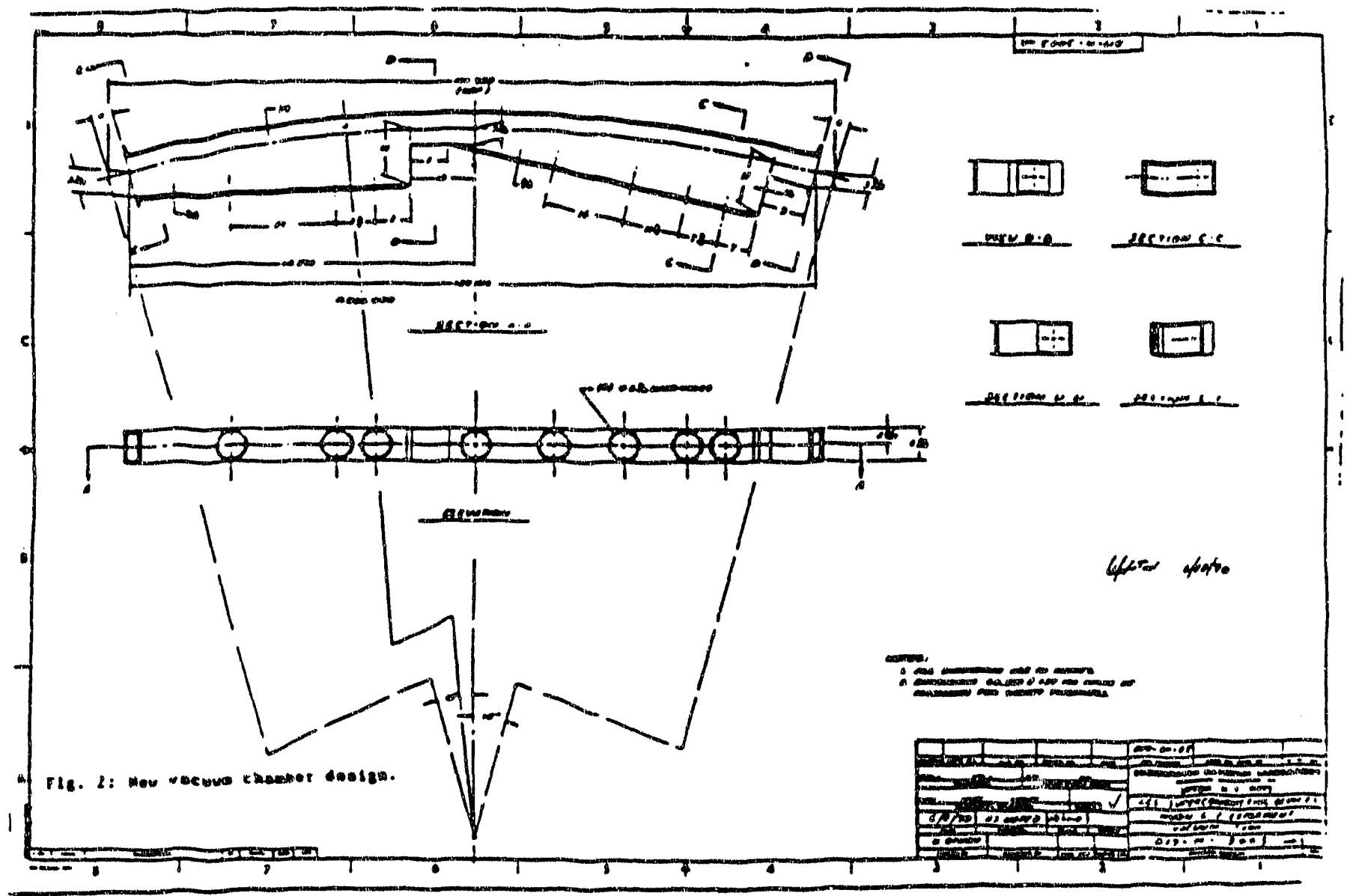


-

-

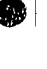

-

D

Poge x-11

Buvitun Uiminetrify 
For Internal Distribution Only

\section{Brookhaven National Laboratory}

Associated Universities Incorporated

Upton, New York 11973

Muon g-2 Note No. 84

Title: The Electrostatic Muon Kicker (17 September 1990 plus ADDENDUM 2 July 1991)

Author: Frank Krienen

Amiliation: Boston University

Date: July 3, 1991 
THE ELECTROSTATIC MUON KITKER

by Frank Krienen

17-SEP-1990 Plus ADDENDOR 2July 1991

The e.s. device bas the advantage of complete cancelation of the effect of lingering eddy currents on the muon population. The required pover per pulse may be less by teast one oreder of magnizude, so ve work on that.

The problem is the very high voltage to deflect, say, $10 \mathrm{mrad}$, or, some $+1-$ $1500 \mathrm{kVmeter}$ on each electrode.

One solution vould be to use Marx generators, however such generators need auch maintenance, axe not sultable for fast cycling, so that the two, we have at BNL, can be at most used for testing purposes, and are as such very velcome. I made then yself some 20 years ago, but they are still going strong.

An altemative vould be to make $40 \mathrm{kV}$ pulses and step those up with a pulse r.ransformer by factor 10 or 30 . The suitching elewent could be a thyratron or a spark gap. It looks that spark gaps would do, as the energy and the charge transport per pulse can be relatively small: 48 joule, respectively 0.0024
Coulomb.

The basie elements of the pulser are show in Figure 1. The crov bar spark gap is left out for the time being.

The preliminary parameters vould be:

$\begin{array}{lll}\text { V(secondary) } & 400 & \mathrm{kV} \\ \text { C(load) } & 600 & \mathrm{pF} \\ \text { Iransformer ratio } & 1:=10 & \\ \text { Pover supply voltage } & 40 & \mathrm{kV} \\ \text { C(charge) } & 60 & \mathrm{nF} \\ \text { R(charge) } & 10 & \text { kOhm }\end{array}$

C(load) lumps together some 4 of 50 ohm electrode. 2 m of connecting cable, feed through capacitance and sowe stray capacilance in the pulse transformer. C(charge) is ade up of 50 obu coaxial cable; some 50 cable loops of 12 w each connected in parallel.

Figure 2 represents the "reduced" scheme, where all elements are relared to the primary side of the transforwer, thus:

$\begin{array}{lll}V-1 & 40 & k V \\ C^{-1}-C_{-} & 60 & n F \\ M^{-} & \text {large } & \\ L & 0.6 & \text { uh }\end{array}$

$H$ and $L$ are the mutual inductance respectively the siray inductance of the transformer. We assume that $K$ is large enough, so as not 10 influence the vollage build up if the suitch $S$ (spark gap) closes. 
Figure 3 shows V 1, V 2 and $i$ under those idealized (no losses) conditions. Thus after half $\bar{a}$ cycIe $C_{2} 2$ is fully charged up, and at that moment the muon bunch will pass through the kicker. After a full cycle all energy would be shifted back into $C_{2} 2$, but the crou-baring should start iumediately after the peak voltage is reached on the load.

Unfortunately ve cannot crow-bar on the priwary side of the transformer, see Figure 4. The cause is the bigh value of $L, j . e .$, the secondary vinding of the transformer cannot be coupled tight enough to the primary winding, due to the high voltage on the secondary terminal. I is estimated to be not less than 0.6 U日, so that the natural undamped period of oscillation is 843 ns. The aspect of the damped output pulse is shown in Pigure 5 for $R=6,3$ and 1.5 ohs. Clearly, the output pulse should be small after some $150 \mathrm{~ns}, 1 . e .$, when the kicked bunch passes for the second tiwe through the kicker. Bence' primary, crov-bar is out of question.

Secondary crou-baring, as shom in Plgure 6, would be more effective. A time constant of, say, 60 ns can be obtained $R=1$ Ohm, or, in secondary units 100 Ohm, giving in Eirst Instance a voltage on the load as shown in Pigure 7 . The Figure needs some further consideration as some energy flows in the transformer, wich eventually would show up as an swall after pulse on the
load.

In this case the burden is on the design of the $400 \mathrm{kV}$ spark gap, but it is probably possible, since the switched energy per pule is 48 Joule at wost and the transferted charge some 0.24 coulomb. Furthermore, the crow-bar spark gap needs to hold the voltage for very small time, -400 ns.

The mutual inductance $A$ should be at lesst 30 times the stray inductance $L$ in order to liajt the magnetization current. It is easy to obtain vith the appropriate ferrite in the core of the transformer. Figure 8 shows a proposed solenoidal design, but perhaps toroidal ferrite rings should be considered as
well.

Thus the final pieture vould be as shown on Figure 9. A more complete urite-up is in the wake. 


\section{ADDENDUM}

The resonant charging of the e.s. kicker occurs at rather low frequency, so that one could envisage a band core instead of a ferrite core transformer. Band cores allow higher fluxes, I $T$ or more, and have much higher permeability than ferrites. Hence one could reduce the overall size of the pulse transformer by, say, a linear factor of two, and reduce the stored magrietic energy dramatically to less than 1 t of the total energy at the time of the crowbar action. This would reduce the tail of the residual electric field seen by the cuasting muons. There will dways be a tail due to the recharging of $C_{1}$, see Fig. 6, but the associated time constant of 134 ns is mueh larger than tae crowbar tima constant, RC = 30 ns, say.

Figure 10 shows a preliminary design of the band core pulse transformer, based on steel made by Vacuumschmelze, Permenorm $36001 \mathrm{K2}$, but any good quality brand would come out equivalent, as the requirements are not excessive. In the design is some mergin to split the core, which would ease the construction. Table 1 shows the data sheet.

The priwaxy switch would be a thyratron, 1.i. English Electric Deuterium fllled ceramic type CX1174C, rated $40 \mathrm{kV}$, $25 \mathrm{kA}$, or type Cx1527, rated $40 \mathrm{kV}, 10 \mathrm{kA}$, which has a metal envelope. As the duty cycle in the g-2 experiment is extremely small, on the order of $10^{-8}$ " the operation should give no problems.

The project needs two decisive tests before one can embark on the extensive design stage.

1. A one meter prototype kicker, to be located in the available vacuum tank and energized by two (available) Marx generators of $250 \mathrm{kV}$ max. each. We contend that if this works, the required voltage of 2 times $350 \mathrm{kV}$ is realizable. This study includes also the feedthrough and the cable connectors, which is a sensitive issue in a magnetic rield.

2. A crowbar sparkgap has to be designed and tested, using the two Marx generators in cascade, so that $400 \mathrm{kV}$ is reached. However, only 48 uC charge is avallable instead of the desired $2.4 \mathrm{mC}$, but it is known here how to dimension the sparkgap for $2.5 \mathrm{mc}$, so, hopefully we may - conclude the foasibility of the crowbar system.

The above tests would be work-intensive but do not require a lot of money to realize. Thus the eirst item for this project is to obtain an engineer/physicist, who is well acquainted with high voltage equipment. 
TABLE 1, Parameter List.

$$
\begin{aligned}
& \hat{v}=40 \mathrm{kV} \\
& C=60 \mathrm{nF} \\
& L=0.6 \mu \mathrm{H} \\
& n=5
\end{aligned}
$$$$
A=16 \mathrm{~cm}^{2}
$$$$
A_{12}=24 \mathrm{~cm}^{2}
$$$$
1_{y}=64 \mathrm{~cm}
$$$$
\left(1_{a}+l_{y} / u_{r}\right)=0.2 \mathrm{man}
$$$$
n_{3}=50
$$$$
\omega=(L C / 2)^{-t}=7.4536 \mathrm{MHz}
$$$$
1 / \omega=134 \mathrm{~ns}
$$$$
T=2=0.8 \text { us }
$$$$
Z=(2 L / C)^{\frac{t}{t}}=4.47 \mathrm{ohm}
$$$$
i=\hat{v} / 2=8.95 \mathrm{kA}
$$

$$
\begin{aligned}
& v_{1}=\{\hat{v}(1+\cos \omega t) \\
& -v_{2}=\hat{f} \hat{v}(-1+\cos \omega t) \\
& v_{L}=\hat{v} \cos \omega t \\
& i_{m}=(\hat{v} / Z)(t+\sin \omega t)
\end{aligned}
$$$$
L=\mu_{0} n^{2} A_{12} / I_{C}
$$$$
l_{C}=0.126 \mathrm{~m}
$$$$
M=\mu_{0} n^{2} A /\left(1_{a}+l_{y} / \mu_{r}\right)=250 \mu H
$$$$
\hat{i}_{m}=x \hat{v} /(2 \omega M)=33.7 \mathrm{~A}
$$$$
\left.\left.\left.B=\mu_{0} n i_{\mathbb{m}} /\right]_{a}+\right]_{y} / \mu_{r}\right]
$$$$
\hat{B}=1.05 T
$$

initially on $C_{1}$

$c=C_{1}=100 C_{2}$

estimated stray inductance ${ }^{\star}$

5 turns on the primary

area of core section

area between primary \& secondary (ave)

flux line length in iron

$1_{a}=$ air gap

secondary turns

resonant charging frequency

char. time constant

full cycle period

charging impedance

peak primary current

voltage across $C_{1}$ voltage across $C_{2} / 100$

voltage across stray inductance

primary current

$r_{c}$ - length of the solenoid

mutual inductance*

current at $t=T / 2$

flux density

flux denisty at $t=T / 2$ 


$$
\begin{aligned}
& W_{\text {total }}(\omega t=0)=\left\{c \hat{v}^{2}=48\right. \text { Joule total stored energy } \\
& W_{L}(\omega t=\pi / 2)=\left\{W_{t}-24\right. \text { Joule energy in stray field (max) } \\
& W_{m}(\omega t-\pi-) \quad 1=0.14 \text { Joule energy of magnetization } \\
& Q=C \hat{V}=2.4 m C \\
& R_{1}=0.0110 \\
& W_{R_{1}}=1 / 4 \text { i } R_{1} T=0.2 \mathrm{~J} / \mathrm{pulse} \\
& W_{R_{2}}=\text { (est.) }-0.4 \mathrm{~J} / \text { pulse } \\
& P_{y}=64 \times 16 \times 8.15 \times 10^{-3}=8.34 \mathrm{Kg} \\
& \text { stored charge } \\
& \text { hf resistance of primary } \\
& \text { ohmic losses in primary } \\
& \text { solenoid losses } \\
& \text { core weight }
\end{aligned}
$$

- with reference to the primary side of the transformer. 
-

FR 900916.3

$\int_{R}^{+40 \mathrm{kV}}$

-

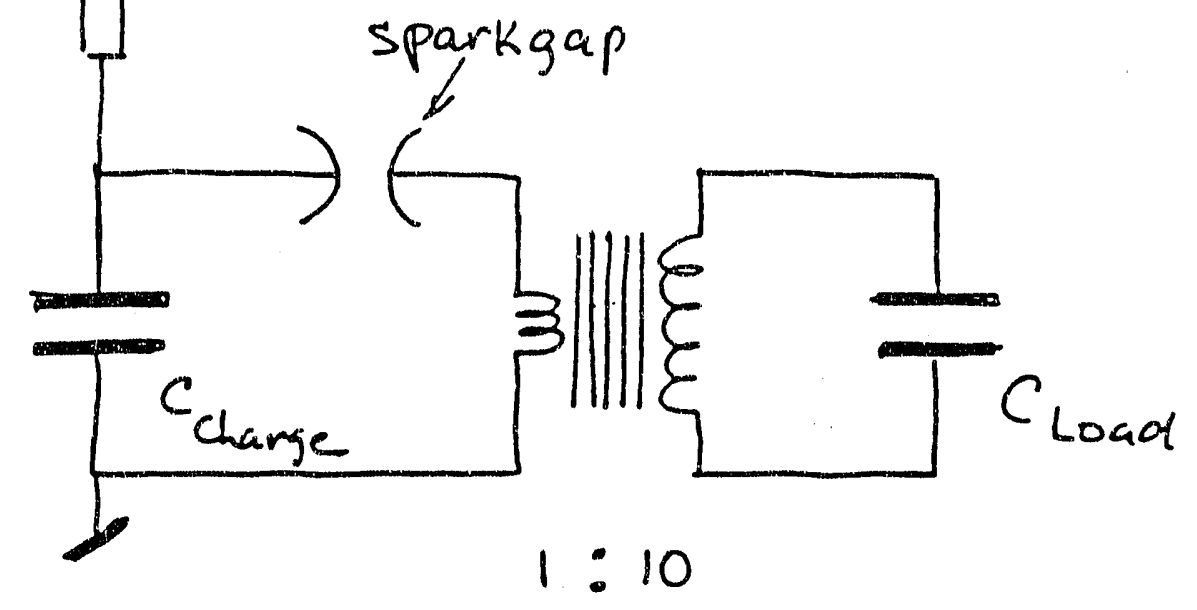

Figure 1

-

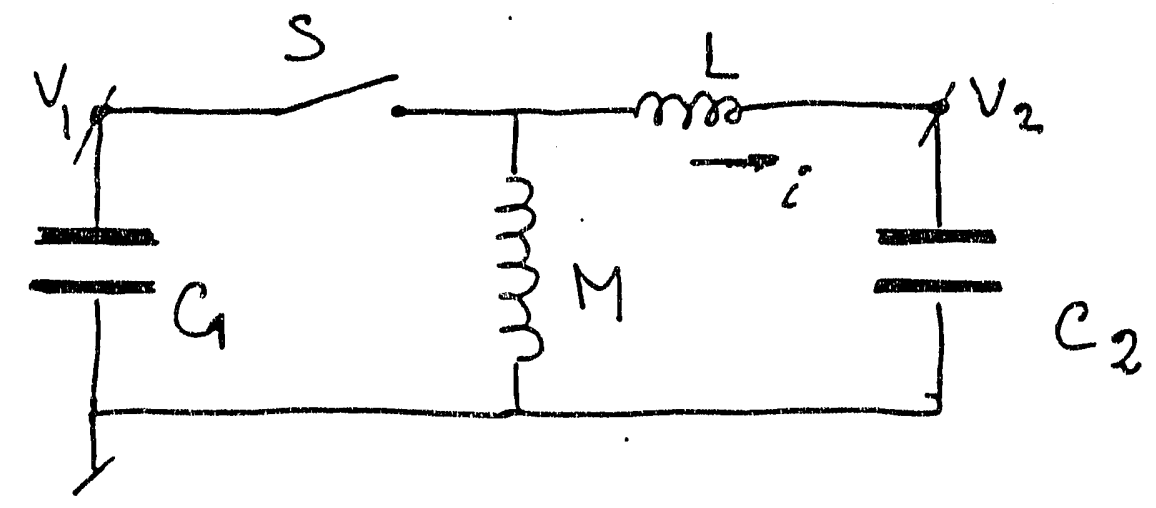

Figure 2

Task B: Accelerator Design Physics: Appendix

Page 2-21

$$
\equiv
$$




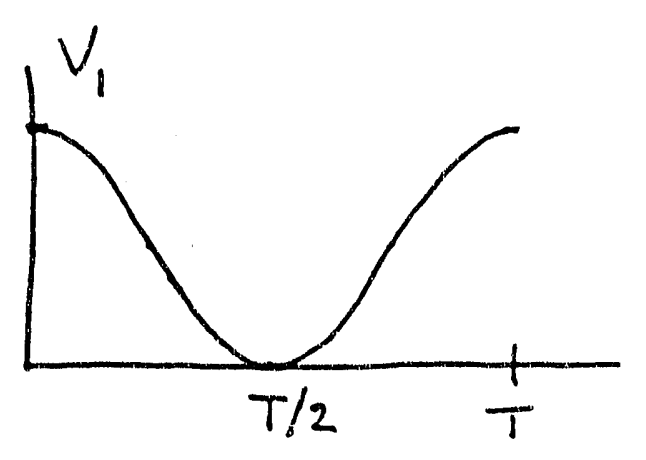

$$
\text { FK900916.7. }
$$
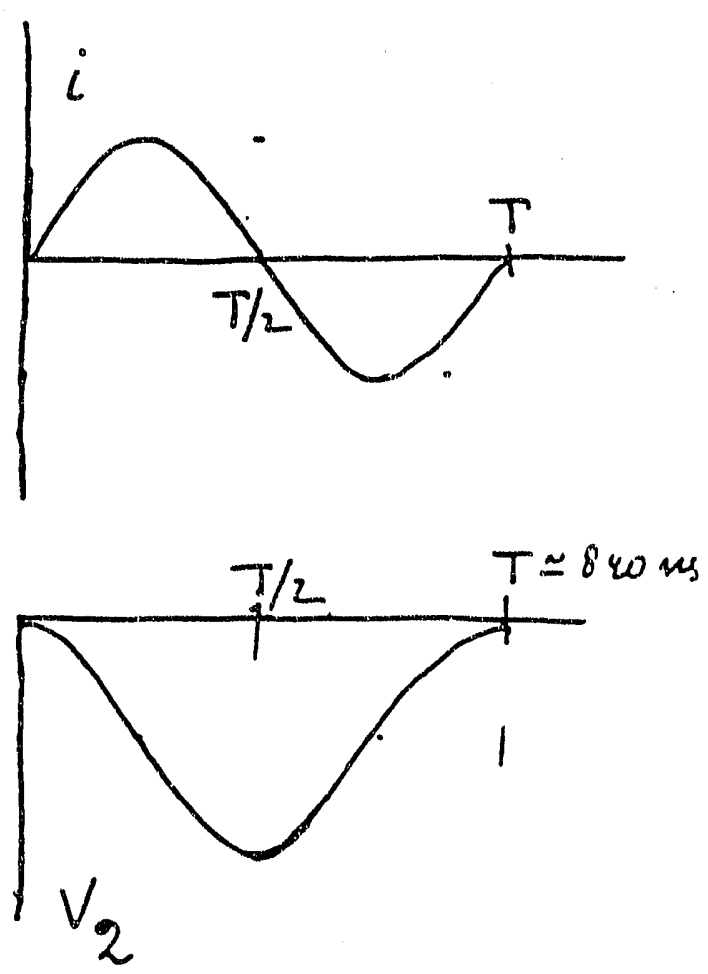

Figure 3

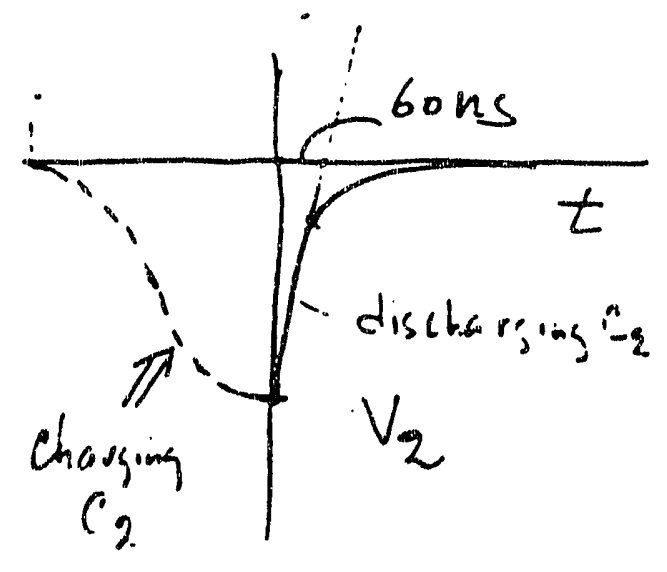

Figure 7.

Page 2.22

Boston University 


$$
\text { FK } 900916.4
$$

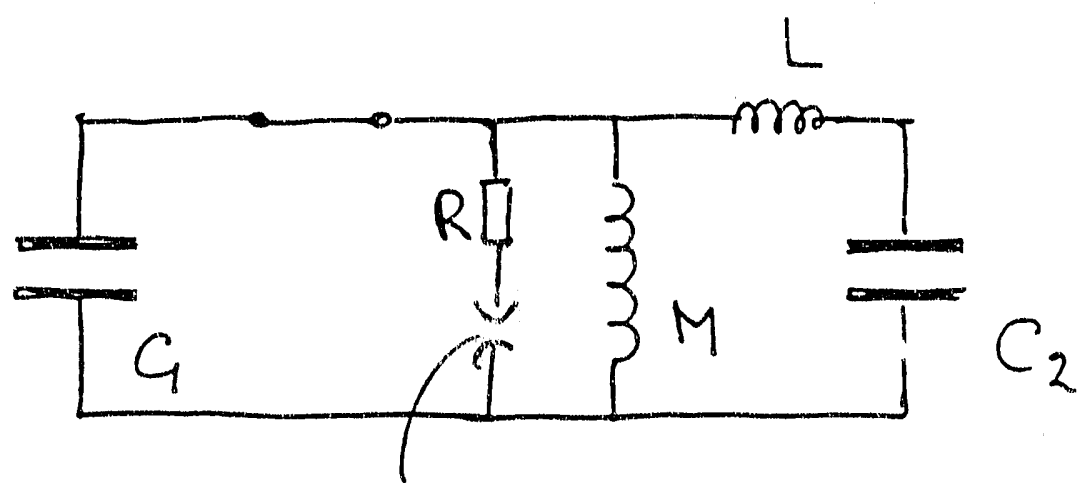

crow-bar,

Fiquie 4

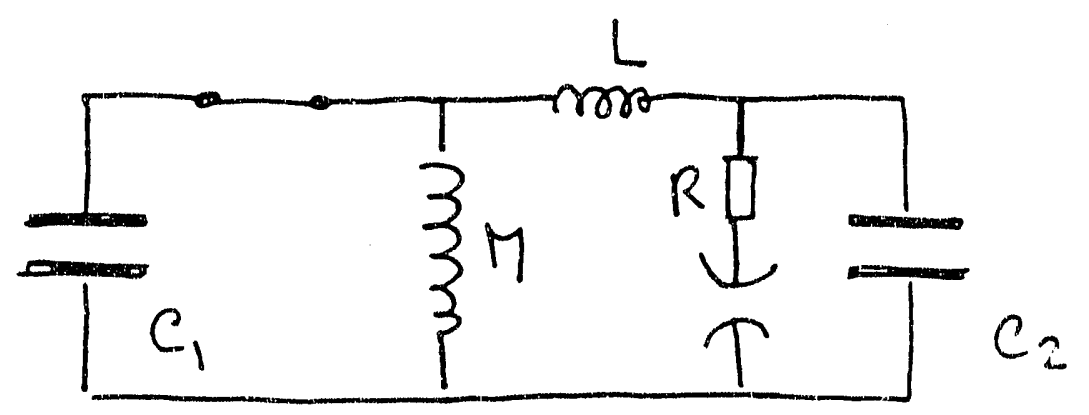

Figure 6

Iast b: Accelerator Vlesign Hhysacs: Appendix

Hage 4-25 

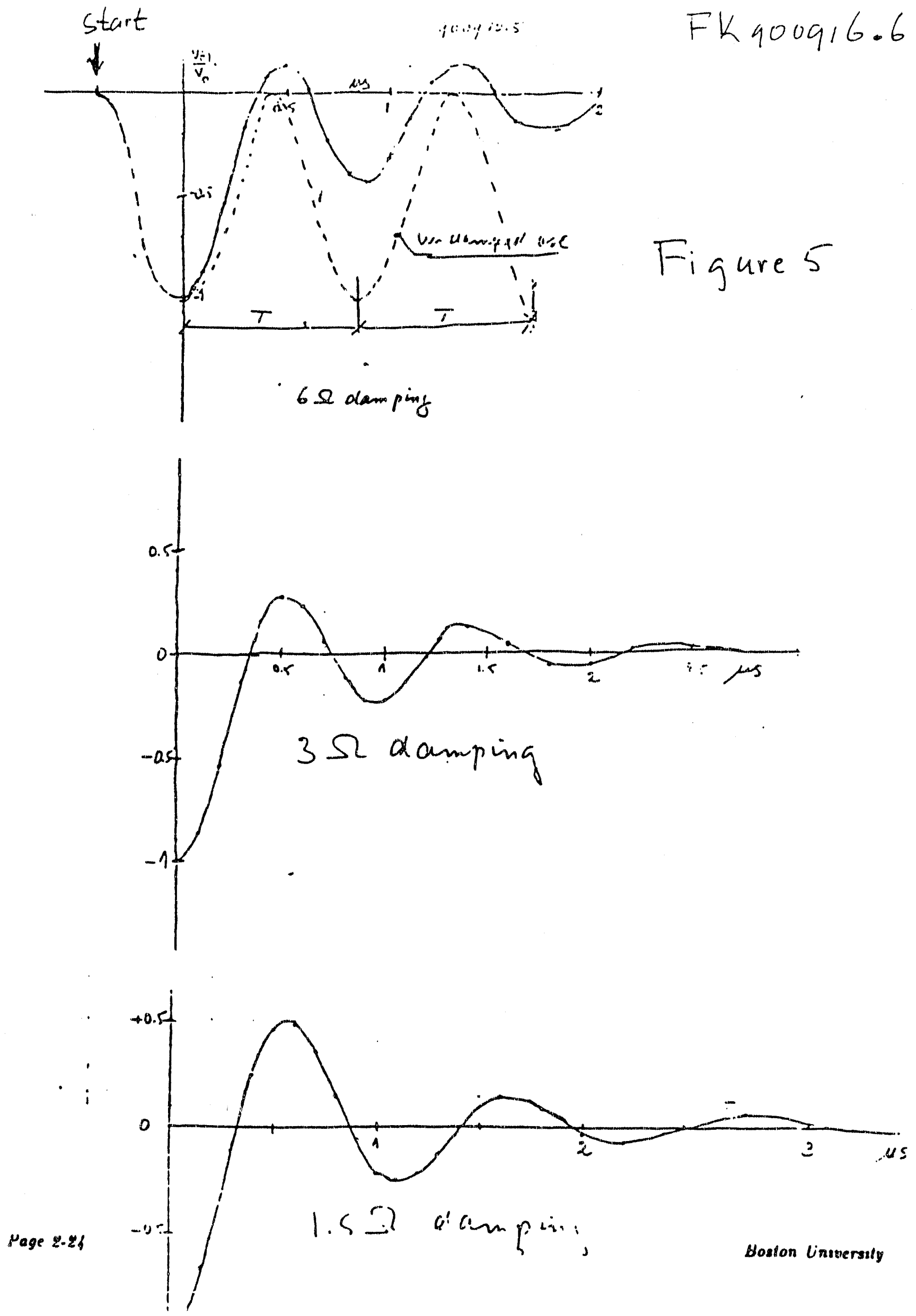


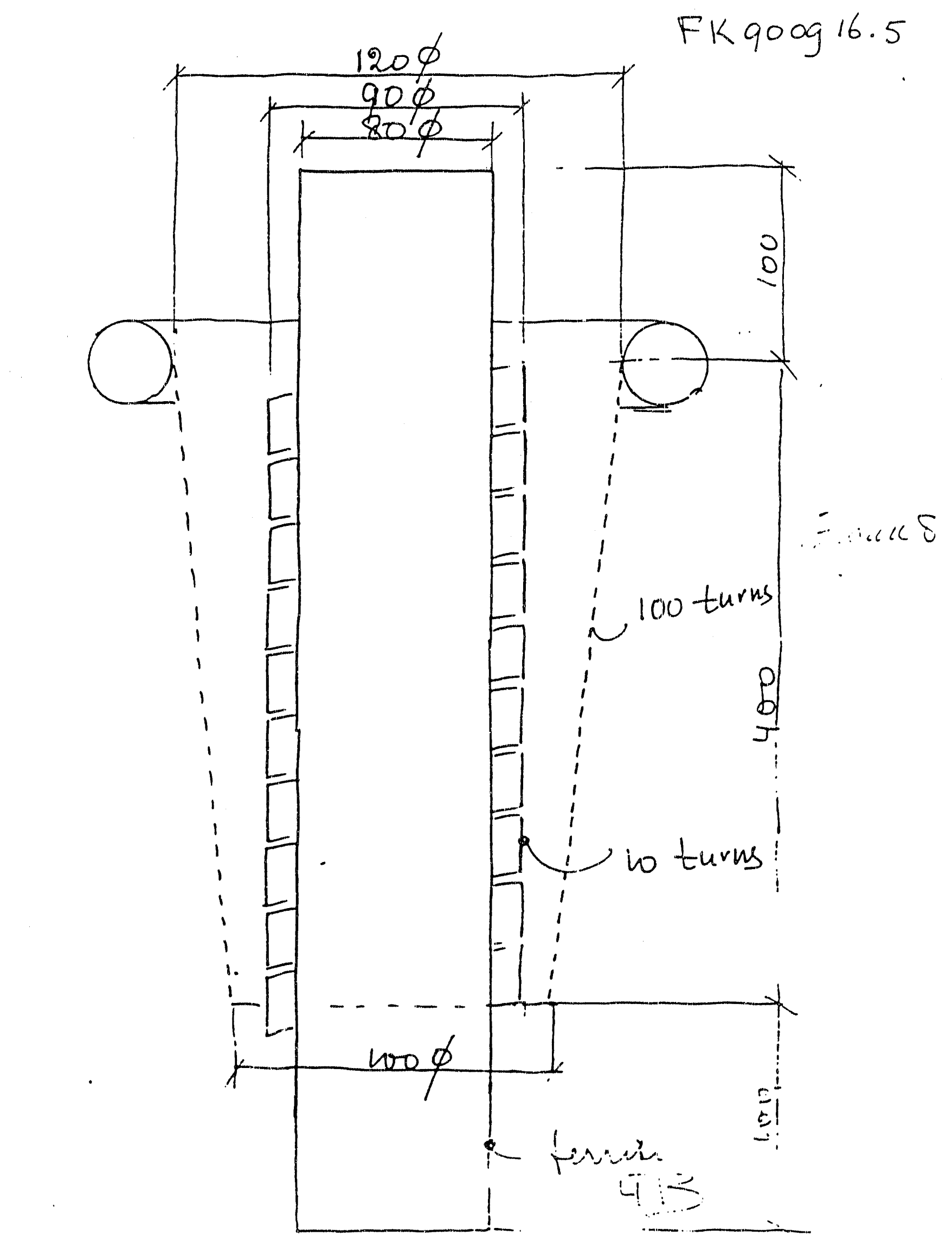

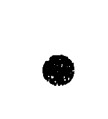

0 


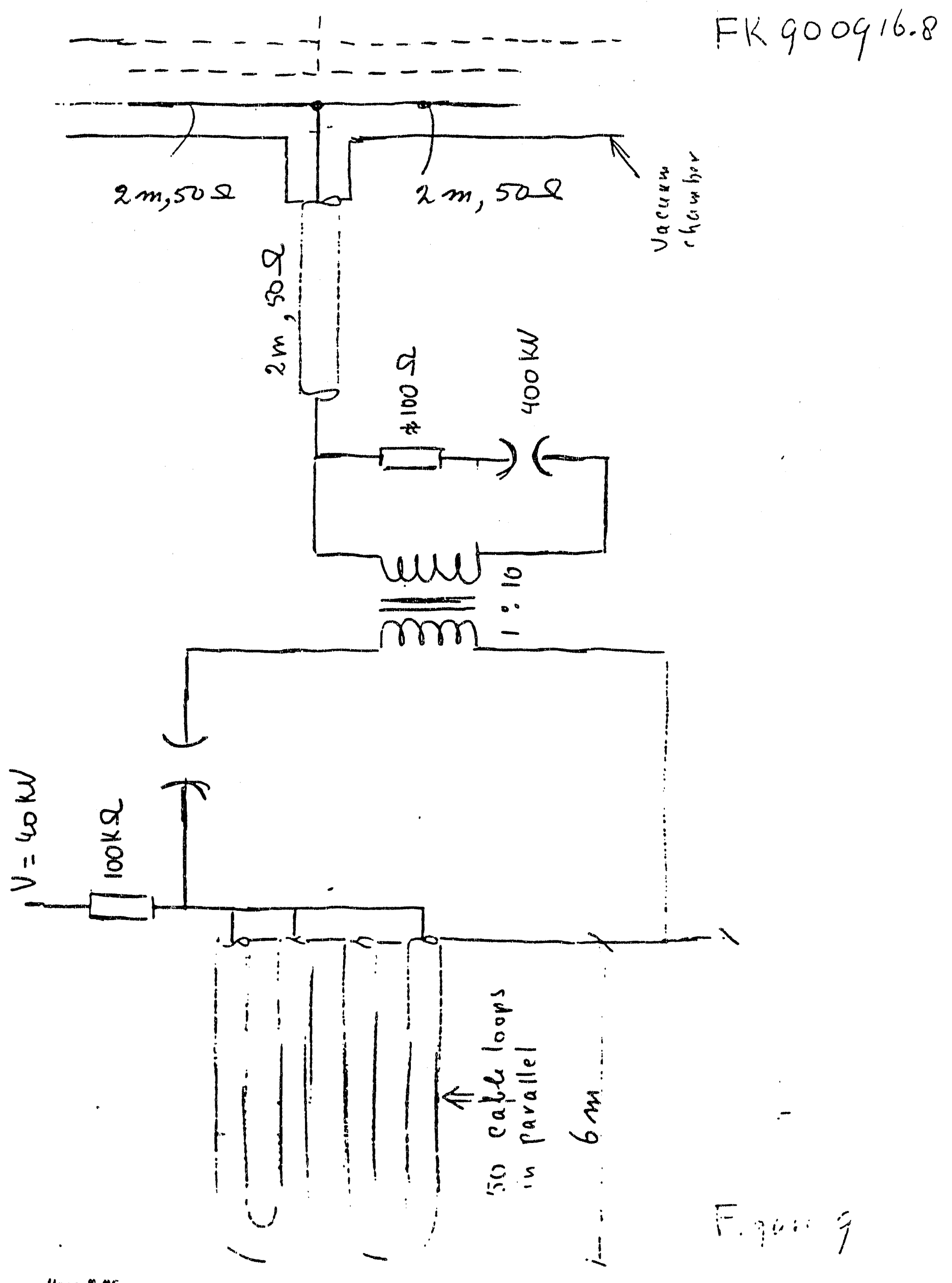


FK 910624.1
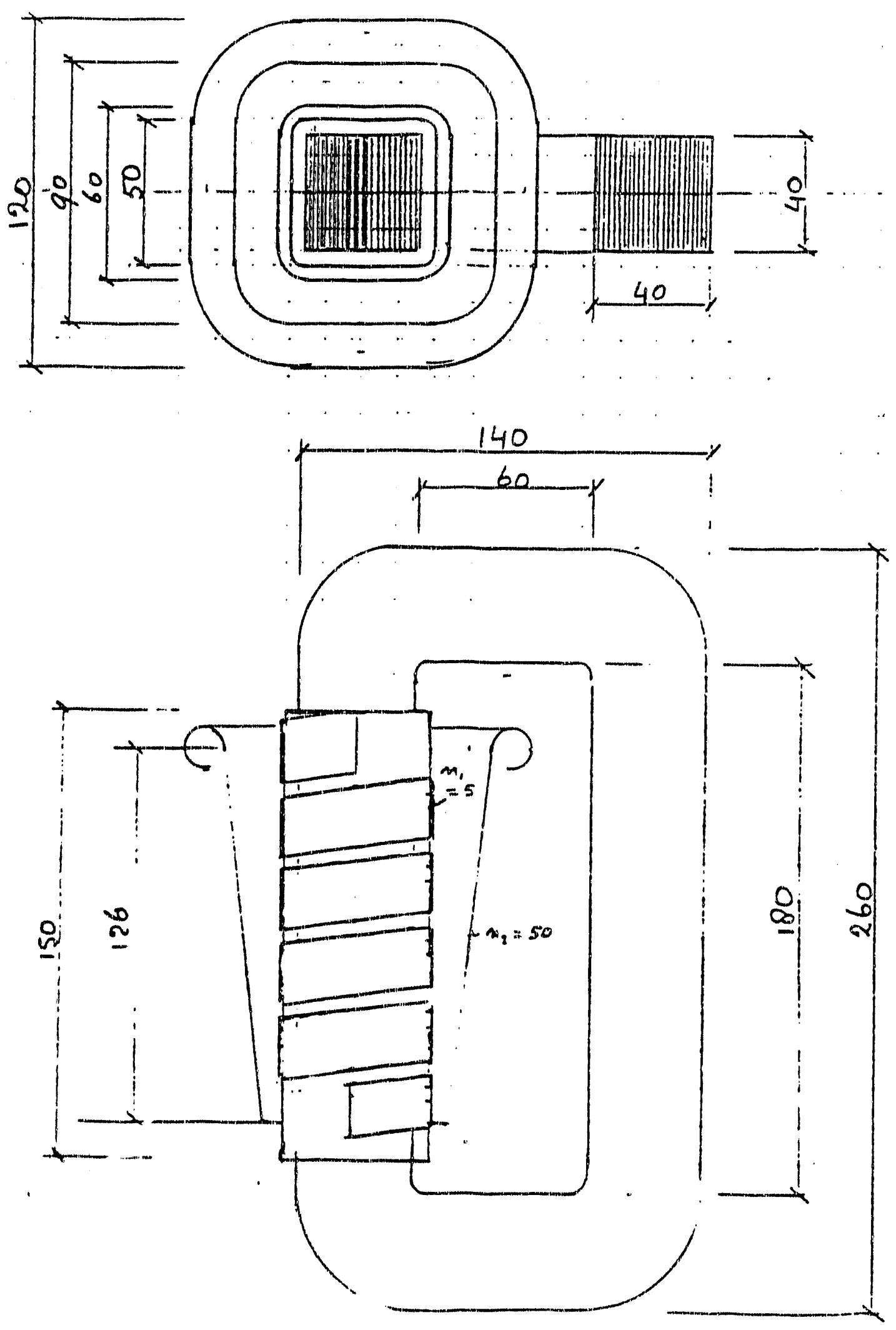
TASK C: PARTICLE ASTROPHYSICS GROUP

Faculty:

Research Associates:

Graduate Students:

Technicians:
Associate Professor James Stone (P.I.)

Professor Lawrence Sulak

E. T. Kearns

J. T. Hong

R. Cormack

G. Ludlam

C. Okada

S. Merritt

H. M. Sun (part time)

\section{Collaborating Institutions}

MACRO Experiment: Bari, Bartol, Bologna, Caltech, Drexel, Frascati, Indiana, L'Aquila, Lecce, Michigan, Napoli, Pisa, Potenza, Roma, Sandia, Texas A \& M, Torino

\section{Introduction:}

The Particle Astrophysics Group has research involvement with the MACRO experiment which dates to its inception in 1984-85. The building of this experiment has proceeded steadily over the past few years in a staged fashion with parts of the detector being brought online for data taking as others were under construction. We anticipate that the full detector will be operational by the end of 1992. The MACRO (Monopole, Astrophysics, and Cosmic Ray Observatory) experiment is located at the Gran Sasso Underground Laboratory near L'Aquila, Italy. It consists of a large area detector dedicated to a search for GUT monopoles and studies of muon and neutrino astrophysics. The MACRO detector is modular and when completed will consist of 6 upper and 6 lower supermodules each measuring $12 \mathrm{~m} \times 12 \mathrm{~m} \times 5 \mathrm{~m}$. The lower supermodules consist of 10 layers of streamer tube separated by $32 \mathrm{~cm}$ of crushed rock absorber. This inner tracking section is surrounded by tanks of liquid scintillator viewed by 8 -inch photomultiplier tubes (PMTs); the scintillator system is the primary responsibility of the U.S. collaborating institutions. The scintillator system performs muon timing and triggering in addition to its main task of sensitivity to 
slow ionizing particles. The upper supermodules form a hollow structure with more tanks of liquid scintillator sandwiched with streamer tubes. This cavity houses the readout elec tronics, and allows for future upgrades such as transition radiation detectors or a solenoidal magnet. As a final redundancy for monopoles and other heavily ionizing particles, plates of CR39 and Lexan plastic track etch detector cover the central plane and one face of the detector. The first supermodule of MACRO has been running since February, 1989. Currently 6 supermodules are instrurnented and will be brought online in the next few weeks. The construction of the upper level of $\mathrm{MACRO}$, the attico, began on November 12 , 1991. It is anticipated that the full detector will be operational by the end of 1992 .

\section{Physics with MACRO}

The idea of the existence of magnetic monopoles dates back to Maxwell's formulation of electricity and magnetism. Then it was seen as a way to symmetrize the Maxwell equations. In 1931, Dirac showed that the existence of a monopole would explain the quantization of charge. More recently, 't. Hooft and Polyakov showed that monopoles are stable solutions of any non-Abelian field theory where a larger group undergoes spontaneous symmetry breaking to produce a $U(1)$ group. In the minimal SU(5) theory, monopoles would have a mass of $\sim 10^{16} \mathrm{GeV} / \mathrm{c}^{2}$ though other theories predict values than span many orders of magnitude around this value. Such monopoles would be expected to be produced in the early universe, and distributed throughout the universe.

Astrophysical considerations place limits on monopole abundances and velocities in the universe. The Parker limit is based on the observed galactic magnetic field, which would be destroyed in the presence of a large monopole flux. For minimal SU(5) and monopole velocities of $\sim 10^{-3} \mathrm{c}$, the upper bound on the flux is $\sim 5 \times 10^{-15} \mathrm{~cm}^{-2} \mathrm{sr}^{-1} \mathrm{~s}^{-1}$. Gravitational and magnetic interactions suggest moriopole velocities as low as $10^{-4} c$. Theoretical and astrophysical considerations suggest an extremely small flux of super-heavy relatively slow monopoles. A search for such particles requires a very large area detector capable of detecting slowly moving, highly penetrating particles.

The large acceptance $\left(\sim 800 \mathrm{~m}^{2} s r^{-1}\right)$ and high resolution tracking of the MACRO defector allows detailed studies of muons from primary cosmic ray interactions in the upper atmosphere. MACRO will provide an enormous data sample of underground muons $\left(\sim 10^{8}\right.$ single muons and $\sim 10^{6}$ multiple muons per year). The single muon data allow for 
searches of cosmic ray anisotropies, time variations, and high energy point sources as well as study of the cosmic ray spectrum. The study of the rates and muon separation in multiple muon events will provide insight to both the primary cosmic ray composition and their interaction with nuclei in the upper atmosphere. In addition, MACRO is collaborating with an extensive air shower array located on the top of Gran Sasso (EAS-TOP), which will provide a unique ability to study coincident events passing through both detectors.

In addition to the primary physics goals, MACRO is sensitive to a variety of possible anomalous effects. The area, depth and redundancy allow for new particle searches for particles with anomalous $d E / d x$ such as "nuclearites" or strange quark matter. Upwardgoing muons from neutrino interactions in the rock below MACRO can be used for searches for WIMPs accumulating in either the earth or the sun as well as for neutrino astronomy. A time-delayed component of muons could indicate the production of long-lived particles in the primary cosmic ray interaction. Measurements of the multiple muon decoherence functions could discover high $P_{t}$ events indicative of massive particles decaying in the atmosphere or new physics in the nucleus-nucleus primary interaction.

The energy released by a gravitational stellar collapse is mostly emitted as $\nu$ and $\bar{\nu}$ of all families during the neutron star cooling phase $(\sim 10 \mathrm{sec})$. The observation of SN1987A by the Kamiokande and IMB experiments largely confirmed the theory of supernova explosion and neutrino emission. Upon completion, MACRO will have a large active mass ( $\sim$ Kton) allowing us to search for stellar collapse events. The detected reaction would be scattering: $\bar{\nu}+p \rightarrow n+e$ where the neutrino has an average energy around $10 \mathrm{MeV}$. The delayed gamma from neutron capture $(2.2 \mathrm{MeV} \sim 180 \mu \mathrm{sec}$ delayed) provides a unique secondary signal that can be detected with $\sim 25 \%$ efficiency assuming a $1.5 \mathrm{MeV}$ threshold. Scintillation detectors are more sensitive to this secondary signal due to their lower energy thresholds than the water Cerenkov detectors. 


\section{Status of the MACRO Detector}

The MACRO detector is being assembled in a staged fashion, which affords us the opportunity to commission the first fraction of it for data taking while assembly continues on the rest. The first major stage was the completion of the first supermodule in February 1989. The data from the first one-twelfth of the final design has already provided for three Ph.D. theses (two from Boston University: D. Ficenec and D. Levin) and several publications and conference contributions (see Bibliography). Most importantly, it has provided working experience from which we can benefit as we assemble and commission the remainder of the detector.

The second major stage for MACRO is the completion of the lower 6 supermodules. The streamer tube tracking for supermodule 2 has been operational since May 1990. Several analyses have been performed using the tracking volume of supermodules 1 and 2 alone. The tracking for all six lower supermodules has been operational since July 1991 . In parallel, the U.S. collaborators have been commissioning the scintillator system for supermodules 2 through 6 . The lower 6 supermodules commenced full operation on August 1. 1992. The upper 6 supermodules of the attico are anticipated to be online by the end of 1992 or early 1993.

\section{Responnibilities and Future Activities of the BU Group}

In the MACRO detector, timing and energy loss information are provided by the signals from the PMTs viewing the scintillation counters from both ends. The BU group is responsible for providing all of the front-end components including the PMTs and associated hardware. This involves the evaluation of candidate PMTs, purchase, and certification of all PMTs $(\sim 1500)$ in the scintillator system. In addition we provide the cables to supply $\mathrm{HV}$ to the tube and to bring the signals to the readout electronics. We are also responsible for the development and production of the PMT base PC board, providing HV distribution to the dynodes and anode readout.

The wide variety of physics being investigated by MACRO necessitates a number of electronics systems, each requiring an accurate copy of the PMT signal. The BU group has designed a fanout module that takes $8 \mathrm{PMT}$ signal inputs and produces 6 output copies of each signal. It is based on the Harris HA3-5002-5 buffer amplifier. Six supermodules of MACRO will require 720 fanout modules. These are being produced by the BU group. 
Because MACRO is searching for extremely rare effects, it is essential to understand the behavior of the scintillator, PMTs and electronics. To this end, BU is supplying the calibration system for MACRO. This consists of two systems. The first is a system of lasers, computer controlled attenuators, and optical fibers to introduce precisely controlled amounts of light into the scintillation counters, providing calibrations of timing and saturation effects. The second is a system of LEDs, computer controlled pulse generators and relays, providing information about timing, PMT gain and trigger efficiencies.

One of the best handles on rare phenomena is the wave form of the signal from the PMTs in the scintillator system. Wave form digitization is essential for noise rejection in searches for monopoles or supernova events. The resolution of the components of the scintillation counters suggests a system that samples at $200 \mathrm{MHz}$, while the desire to keep the data rate manageable makes zero suppression essential. Also, one must be able to record signals ranging from $3 \mathrm{mV}$ to $10 \mathrm{~V}$. This will be accomplished with a custom fast wave form digitizer (FWFD) that has been conceived, developed and produced through the prototype stage at $\mathrm{BU}$. Work on this project will continue at $\mathrm{BU}$ in collaboration with other members of the MACRO-USA collaboration.

Below we provide detailed descriptions of our hardware and software responsibilities for the MACRO experiment.

\section{PMT Related Activities}

Before installation in the detector, all PMT's must first be certified at BU. MACRO currently uses an eight-inch PMT, model number D624KB, manufactured by Thorn-EMI Electron Tubes Limited. Upon arrival, each tube is visually inspected, and tube parameters (as measured at the factory) are archived. Each tube is subsequently put through a series of measurements to evaluate its performance. The most extensive of these is a measurement of P.IT gain as a function of voltage and illumination. The gain map is then used to detcrmine operating voltage for measuring dark noise, pre-pulsing, after-pulsing and timing variation. All of these parameters are necessary to certify a PMT, and to identify paired tubes for installation in the detector. This procedure has been completed for the PMTs of the lower modules of MACRO $(\sim 950)$, and we will continue to test tubes destined for the attico $(\sim 750)$. 
A number of other hardware responsibilities are associated with the PMTs, e.g., the development and production of the PMT base. Base development was carried out at BU while manufacturing has been carried out by a local company with quality control done at $\mathrm{BU}$. Production will be necessary for the attico, though a change to a new PMT would also require a new base development. Cable $(\sim 1000)$ production will also be done at $B U$, as well as HV resistive dividers $(\sim 350)$ required by the $2: 1$ multiplexing of PMTs to HV supplies.

\section{Signal Fanout}

The signal fanout is a crucial piece of electronics being produced at BU. Due to the variety of phenomena for which MACRO is searching, there are multiple trigger systems, each of which requires a copy of the PMT signal. The signal fanout carries out this function by providing 6 identical copies of each PMT signal. The large dynamic range of the PMrT signals, and the high sensitivity of the monopole triggers, mandated a careful design to insure that cross talk is below $0.5 \%$. The evaluation of various components and design of the module was carried out at BU. The design is implemented on a $6 \mathrm{U}$ VME boards, cach board fanning out 8 PMT signals, with custom designed backplanes. Care was taken in the design to insure that the LED calibration system (below) could use the same crate and backplane configuration. Production and testing of modules for the attico continues for the coming period.

\section{Laser/Fiber Optic Calibration System}

Based on the experience with the first supermodule, many improvements were made on the design, implementation and development of the calibration system and associated clectronics, software and data analysis. This includes new techniques to produce the optical fibers, and a new computer controlled optical attenuator system.

The laser calibration system developed and tested on the first supermodule is working well, being used by the entire collaboration for the calibration of various subsystems. ADC pedestals and slopes, TDC offsets and slewing corrections, phototube saturation and photoelectron calibrations are performed with the laser system by American groups for IMIU and ERP ADC/TDC systems and by the Pisa group for the PHRASE subsystem. We also have been involved in the software developrnent in association with the calibration 
of scintillator data. The laser system test setup at BU was also instrumental in development of the prototype fast wave form digitizer.

Figure 1 shows the schematic of the laser/fiber-optic calibration system for all supermodules. The first supermodule is different only in the number of fibers (3) in each scintillator tank, but it will be reconfigured as the other supermodules in the near future. During the 1989 run we found that a simpler and less costly system based on one fiber per tank can do the calibration as adequately, with the saved money being used to improve the automatization of the calibration system. The laser UV light $(337 \mathrm{~nm}$, $40 \mathrm{KW}$ peak power, FWHM = $2 \mathrm{nsec}$ ) is collimated into a high power fiber, attenuated by a computer controlled optical variable attenuator and split to all tanks from one supermodule. Each tank has an optical fiber mounted in the middle of the tank.

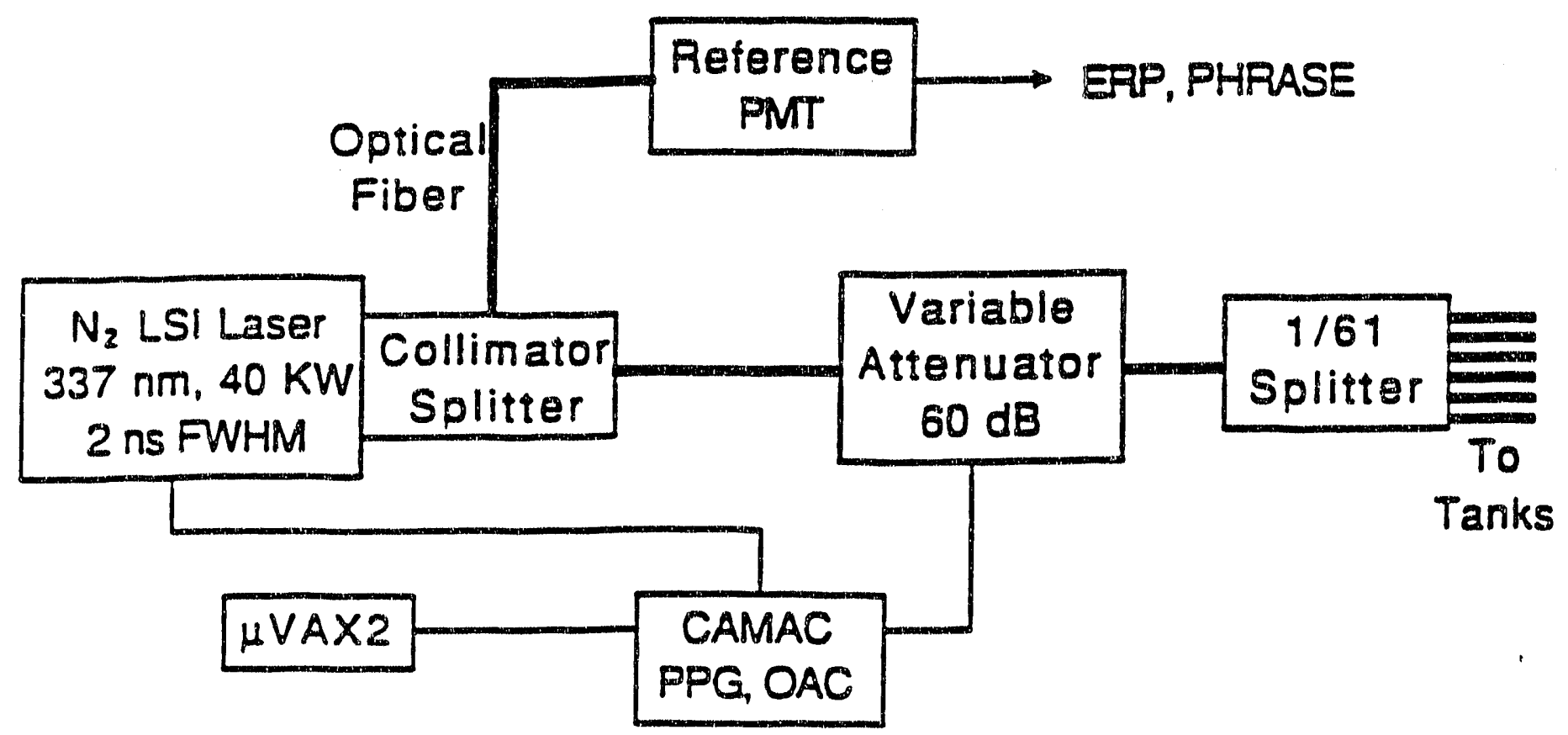

Fig. 1. Layout of the Laser/Fiber Optic Calibration System 
The laser/optical fiber system is currently driven by the same VAX which runs the online system, but using a separate, independent program, which also drives the computer controlled optical attenuator. The calibration data taking during the normal runs (the online calibration) is switched on every 4 hours for a short period of time, and special calibration runs (the offline calibration) are performed once per week. A reference PMT, used for pulse to pulse correction for the laser jitter, improves the quality of the calibration data base by eliminating the short term laser jitter and the dependence of the average laser output on time.

As of the end of 1991, the laser calibration system was fully implemented in the lower supermodules 2 through 6 . Along with the LED calibration system, this provides all the calibration needs for the scintillator system of the MACRO experiment.

\section{LED Calibration System}

On the first supermodule of MACRO, the LED calibration system was designed to send an identical signal to an LED in each scintillator tank. This was achieved using a PCcontrolled Programmable Pulse Generator (designed and built at Boston University) going into custom-built fanouts (also designed and built at Boston University). This was ideal for doing TDC calibrations and for exercising the monopole triggers via the production of very wide signals. When the time came for the upgrade to six supermodules, the Boston group) looked very carefully at the system and realized that much could be done to improve it.

MACRO is a detector which searches for many rare and/or hypothetical particles and events. If such particles or events are not found, flux limits are set. These limits are functions of angle and particle energy or velocity and are directly related to the trigger efficiencies of the electronics. The BU group realized that the LED system could be generalized to a hardware system that could directly measure these trigger efficiencies and, in fact. measure the efficiency of every aspect of the detector, from phototube gain to electronic triggers to analysis routines. Such an efficiency measurement, when coupled with the detector live-time, is essential in determining flux limits in the absence of an observed signal.

The primary idea of the LED system was to directly simulate the scintillation light produced by a particle traversing the detector. The most useful and general interaction 
to simulate (while keeping the system from being too costly and unwieldy) is a two-tank particle interaction. (This can be upgraded to a three-tank interaction when the attico is built.) This simulation requires the ability to generate a pulse of light of variable pulse height, width and delay independently at each scintillator tank end, while NOT pulsing any other LED in the system. It also must be possible to generate such a simulation for any pair of tanks in the detector for which it is geometrically possible to have a iwo-tank interaction. This led to the conception of a computer-controlled system of pulse generators, fanouts and relays, which could fire any combination of LEDs in the entire detector with four independent pulses. (Note that we are still left with the capability of firing every LED in the detector with the same pulse for the general TDC calibrations mentioned for the first supermodule.)

For this purpose, the Boston group designed and built the MACRO LED Switchbox. This is a combination fanout and relay system, modules of which are mounted in the same custom-built VME crates designed for the BU PMT signal fanouts. Each module sends signals to eight LEDs in a particular face of one supermodule and is controlled by a 24 bit dataway genernted by a CAMAC output register, which receives write statements from the microlAX appropriate to a particular supermodule. The data bits are distributed as follow's: 8 to turn on or off a channel, 8 to switch or not switch a relay in a channel, 7 to address a module, and 1 to strobe the system. Every tank has an LED at each tank end (defined as ends 0 and 1), and the symmetry of the detector with respect to tank ends is exploited so that only two pulses need to be distributed to end 0 LEDs, and an independent set of two pulses is distributed to end 1. LEDs. Thus, there are two pulser inputs at the front panel of each module, and eight outputs come out at the backplane. Thus, two VME crates with 10 modules per crate are sufficient to operate one supermodule, including the attico.

The pulses are generated by Hewlett Packard Pulse Generators. They are controlled by the microVAX via a GPIB CAMAC Interface. The pulses have variable rise and fall times down to 5.5 nsec and have variable heights, widths, and delays. Each pulse generator has two outputs, so that two such pulse generators (with local fanouts) are sufficient for the entire detector. For future applications, the two output pulses can be superimposed to give a more complicated wave form. A block diagram of the LED calibration system for one supermodule is shown in figure 2. 


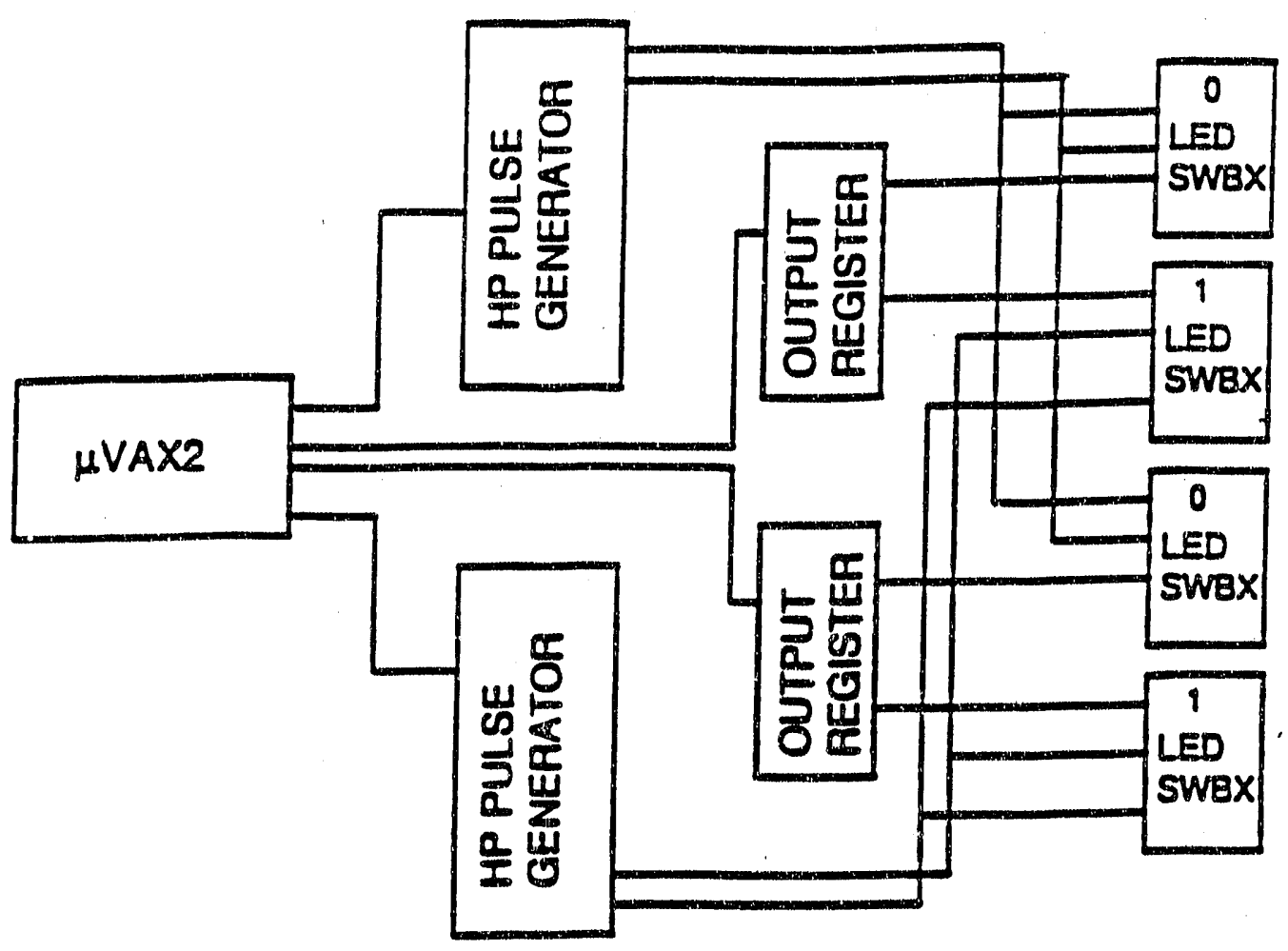

Fig. 2. Block diagram for the LED calibration system.

In order to accurately simulat a particle such as a monopole, the response of the LEDs in the system must be known in terms of number of photoelectrons at the photocathode of the phototube as a function of height and width of the pulse sent to the LED. If this parameterization is done in terms of the pulses generated at the pulse generator, then any non-unity gain in the LED Switchbox is also taken into account. Empirically, we have found that the number of photoelectrons can be fit very accurately by a second order polynomial in pulse height and linearly in width with an exponential turn on (due to finite LED rise time). These parameters are measured for each LED in the system, and a lookup table is generated which allows one to go from desired light at the photocathode to appropriate pulse parameters.

The generation of a typical simulated particle goes as follows: The online system runs an independent calibration program. That program first selects from the desired distribution (whether isotropic flux or distribution designed to test each electronics channel with equal statistical accuracy) a particle at random. The LEDs to be fired are chosen, and channels are turned on and relays are set appropriately. From the parameters of this particle, the phototube pulse at each tank end is reconstructed in terms of timing 
and numbers of photoelectrons at the photocathode. Using the look-up tables, pulser parameters are generated and the pulsers are set up through the GPIB interface. Finally, the pulsers are triggered and the simulated particle is generated. The VAX system is used for these calculations and communications, but programs independent of the online system are used, so that the online system is left ready to either trigger or not trigger on the event, without interference from the calibration routine.

This system has been completed and installed by the Boston University group, and it is currently running on the MACRO detector for the lower 6 supermodules. The system is versatile enough to be prepared for such upgrades as the simulation of three-tank interactions when the attico is completed. But the full potential of the system is continuing to be realized as collaborators with different physics goals contribute the software for the generation of such events as fast and slow monopoles, fractionally charged particles, stellar collapse, muons, upward-going muons, etc.

\section{Fast Wave Form Digitizer Development}

The fast wave form digitizer $(200 \mathrm{MHz})$ system is one of the last major electronics systems needed for the MACRO experiment. The purpose of this system is to record full wave forms at very high sampling rate for the various types of signals expected. The FWFDs are a key component in our search for slowly moving magnetic monopoles. Recognizing the urgent need for this critical circuit, the BU group initiated a design and development effort. A circuit was developed, tested via a CAMAC prototype, and is currently undergoing its final revisions and layout for VMEbus operation. The final system will be multiplexed 2:1 with 4 input channels per VME module. Here we describe the basic principle of operation of the FIVFD system.

Circuit operation is most easily separated into two parts, the high speed digitization . process and VMEbus operations. For the most part these are independent in that VMEbus operations are not possible while digitization is occurring and vice-versa.

Digitization for a single channel begins with the four signal inputs being equally summed into a common output signal which is then amplified and shaped in a nonlinear fashion. The nonlinear output signal is then supplied to a flash $A / D$ converter as a signal in the range of 0 to -2 volts. The flash $A / D$ converter is driven by a $200 \mathrm{MHz}$ system clock and is always performing conversions whether or not the data is being saved in RAM. One 
of the outputs from the flash $A / D$ is a data-ready signal indicating the presence of valid eight-bit output data. This signal is used as the basic clock signal by the ASIC associated with the channel.

The primary function of the ASIC is to transform data appearing once every five nanoseconds into parallel data which can be stored in RAM once every 20 nanoseconds. To do this, the sample and threshold data are sequentially shifted through a series of four registers at a $200 \mathrm{MHz}$ rate. Thus, at any instant of time, registers in the ASIC contain the four most recent data samples and their associated threshold conditions.

If digitization is enabled, then all of the threshold bits for the four most recent samples are examined for non-zero values. Any non-zero bit initiates a sequence of operations via control logic. The first operation performed by this logic is the parallel strobing of the four sample values, the four sets of corresponding threshold bits and the sixteen-bit time word into eight eight-bit latches. The data in these latches remains stable for the next 20 nanoseconds, during which time it $i$; transferred to a second set of latches and finally stored in appropriate RAM locations. The RAM address, also generated by the ASIC, is sent to external registers at this time. A shift register within the ASIC provides the signals for strobing the data and address information into these external registers and for generating the appropriate write pulses to the RAMs.

The VMEbus function is rather complex. Some of this comes from the need to have block transfer capability (for rapid readout of signals). Other complexity comes from allowing VMEbus to perform both write and read operations on the RAM in order to allow for memory testing.

The VMEbus addresses the RAM of each four channel circuit as a block of $256 \mathrm{k}$ bytes requiring 18 bits of address, the upper two bits used to select one of the four channels. A five bit switch allows the address of each waveform digitizer to be moved in blocks of $256 \mathrm{k}$ locations. This switch is intended to be used to set the block address to correspond to the slot number in the crate. An additional 9 bit switch allows the overall address to be moved in steps of $8 \mathrm{M}$ bytes over a range of $4000 \mathrm{M}$, the full 32 bit address range of VMEbus. All types of VMEbus data access are allowed, that is, single, double, triple, or quad byte operations. For block transfer operations, up to 256 bytes may be read or written at a time, but using only the lower eight address bits. The details of VMEbus operation involve the interaction of several control signals with rather stringent timing constraints. 
Currently, the ASIC is fully designed and under production. The VMEbus circuitry is designed and under evaluation. The first prototype PC board has been produced and will undergo testing at the detector at Gran Sasso Laboratory in August 1992. Assuming no substantial modifications in design or layout, production will begin immediately, to be completed by early 1993. The manufacturing, testing, and implementation of the final FWFD system will be a joint effort involving Texas A.\&M., Caltech, and Sandia in addition to $\mathrm{BU}$.

\section{Data Analysis at $B U$}

To take advantage of the staged running of the MACRO detector, the Boston University group is continuing its analysis program with the recent data sample acquired from May 1990 to February 1991. This data consists of two supermodules of tracking detector and one supermodule of scintillation detector. Current Boston University students are slated for thesis topics using six-supermodule data, but the available data affords a good opportunity to develop algorithms and produce preliminary results. The data from Fobruary 1991 to present represents the turn-on of supermodules two through six. The snalysis of this data will emphasize the calibration and shake-down of the new PMTs and electronics.

\section{DST Production}

The first step for much of the analysis is to process the data through the standard production package and provide a summary convenient for analysis. A group has formed, with Ed Kearns as a member, to assemble a DST which is generally useful for muon physics analysis (muon astronomy, upward muons and multiple muons). The first version of this DST, consisting of the two-supermodule data sample, has been available since May 1991.

The muon analysis DST is produced on VAX workstations at the Gran Sasso Laboratory. The information consists primarily of a summary of global event information (such as the Liniversal Time of the event), the reconst ructed track directions, and the scintillator raw data. Because the tracking algorithms are very $C P U$ intensive, it provides the benefit that each person is not separately using limited computer resources to obtain the same information. Also, the amount of information is reduced to approximately 500 bytes per event. This allows us to keep the entire data sample on disk. Thus, analysis of the entire 
sample of 700,000 events takes approximately two elapsed hours of VAX 3200 time, with no tape mounting required.

The first version of this DST production was optimized for muon astronomy; in fact, it was used for astronorny analysis presented in our ICRC papers. 't has also been used by Ed Kearns for tracking resolution and streamer tube geometry studies; by Colin Okada for atmospheric muon analysis; and by Robert Cormack for exotic dimuon analysis. The latest version, available since June 1992, incorporates feedback. from groups interested in multiple muons and neutrino-produced upward going muons. As the detector reaches its next stage of implementation, the quantity of data will preclude storing more than a few months of running on disk. However, we have set up the production so that the DST is arailable immediately after the data is acquired. This will give us very fast turn-around on analyses such as looking for anomalous muon bursts from Cygnus X-3.

\section{Atmospheric Muon Flux}

Lintil MACRO finds its first monopole, effectively every ionizing particle to pass through MACRO can be assumed to be a muon. And the vast majority of these are produced by primary cosmic rays striking nuclei high in the atmosphere and initiating a hadronic shower. Colin Okada has recently begun analysis that studies the flux of muons underground. In particular, the object is either to detect or set a limit on the production of prompt muons produced by high energy cosmic ray interactions.

Niost atmospheric muons are the result of the decay of charged pions and kaons that are in the hadronic shower of the primary cosmic ray. A complete transport calculation reveals two important features for the spectrum of "ordinary" atmospheric muons: the energ: spectrum is a power law, $E^{-\gamma}$, where gamina is approximately 2.7 , one unit steeper than the primary cosmic ray spectrum; the intensity has a $1 / \cos \theta$ dependence caused by the competing effects of interaction and decay. Another component of the muon flux, which has yet to be definitively observed, is the result of the decay of charmed (and beyond) mewons. Because these are very short-lived particles, the effect of interaction does not comprete with deray and the intensity does not depend on polar angle. Also, the energy spectrum is as flat as that of the primary cosmic rays.

Because MACRO is very deep underground, muons must be very energetic at the Earth's surfuce (>1.4 TeV) to reach the detector. However, the overburden of the MACRO detector is the very irregularly shaped Gran Sasso mountain. Therefore, in different direc- 
tions the muon flux varies by an order of magnitude: high where the mountain is shallow, low where the mountain is thick. The search for prompt muons uses this to separately measure the integrated intensity at different polar angles and cut-off energies. We attempt a global fit to a combined theoretical intensity containing a non-prompt component that falls steeply with energy and $1 / \cos \theta$ and a prompt component that is flatter with energy and is isotropic.

So far we have determined that the limiting factor seems to be the systematics of the determination of the overburden. This includes the actual slant-depth to the surface as well as the density of the rock along that path. These are needed to compute the survival probability for a muon of a ceriain energy to reach our detector. We will be working to reduce these systematics. In the interim, this analysis is providing a useful tool for studies such as estimating our certainty of the absolute detector orientation with respect to the mountain.

\section{Dimuon Studies}

Currently there is a feeling that the standard model of particle physics, as successful as it may be, is inadequate. Theorists have suggested a number of improvements such as supersymmetry or composite models. Searches of these new theories will be carried out at the SSC. However, cosmic rays offer interaction energies well above those available at accelerators. Robert Cormack has recently begun an analysis of dimuon events in MACRO as a search for physics beyond the standard model.

SUSY' predicts the existence of a supersymmetric partner to all currently known particles although the mass of even the lightest superparticle (LSP) is not attainable (or known) at current accelerators. It is possible that LSPs produced in primary interactions in the atmosphere would produce dimuons with a vertex in the vicinity of MACRO. A study of these events could indicate the presence of new weakly interacting massive particles. The main background to this will be multiple scattering of muons in 'normal' dimuon events.

Composite models predict the onset at high energy of a constant term in the quark. quark cross-section. This would appear as events with unusually high $P_{t}$ which in turn would appear in MACRO as multiple muon events with unusually large separation. This is separate from the measurement of the decoherence function which would provide information on the cosmic ray composition. The $6 \mathrm{SM}$ of tracking recently turned on provide a probe up to $72 \mathrm{~m}$ separation, a region previously unexplored. 


\section{Magnetic Monopoles}

When MACRO reaches its next stage with six supermodules operating, we will reach a drastic increase in sensitivity to rare particles such as magnetic monopoles. Gary Ludlam, a third-year student, intends to do his thesis analysis using the first six supermodules operating with an interim wave-form digitizing scheme. Gary has been developing the LED farsout system (described above) which will be essential for simulating monopole events in the data stream. Frank Welte, currently a summer student, is also interested in monopoles for his thesis; he will have the opportunity to work with the $200 \mathrm{MHz}$ WFD system, both for hardware experience and as the source for his analysis.

The magnetic monopole is expected to be a cosmological remnant of the Big Bang. It is also a candidate for solving "the Dark Matter Problem". Grand Unified Theories predict super-massive $\left(\approx 10^{16} \mathrm{GeV} / \mathrm{c}^{2}\right.$ ) magnetic monopoles but do not predict the abundance. Because these particles are very mp $\cdots$ we must account for the possibility that their velocity may range from the speed .. light down to as slow as we can experimentally reach. Simple cosmological models predict a great abundance of monopoles; inflationary models make no firm prediction. Past experiments have already shown that the monopole is relatively rare. A reliable limit on the flux has been calculated based on the persistence of galactic magnetic fields (the Parker Bound). Therefore, the MACRO experiment has been designed to be very large and run for a long time, so as to probe fluxes beyond the Parker Bound of $10^{-15} \mathrm{~cm}^{-2} \mathrm{~s}^{-1} \mathrm{sr}^{-1}$.

The MACRO search will rely on the redundant detector capabilities of our scintillator system, charge-readout of the tracking detector and a passive system of track-etch detectors. The Boston University contribution will center on the scintillator analysis. The scintillator system has a 3 -fold redundancy for monopole detection. We require that a monopole cross two layers of liquid scintillation counters. During the traversal of each counter, a slowly moving monopole is expected to produce trains of single photoelectron pulses commensurate with its velocity. These will be recorded with the FWFDs. A candidate monopole must have consistent wave forms in 2 layers of scintillator as well as a consistent flight time between the scintillator planes. Hence, the 3-fold redundancy. For all candidates found, additional confirmation may be obtained from the streamer tube tracking system (Drell-Penning effect) and the track-etch plastics. 


\section{Budget Discussion}

The salary support for our program includes direct support for Profs. Stone ( 2 summer months) and Sulak (2/3 of a summer month); two Research Associates, Ed Kearns (12 months) and Jiangtao Hong (12 months); one technical staff member, Sean Merritt (12 months); three graduate students, Robert Cormack (fifth year), Gary Ludlam (third year) and Colin Okada (third year); and a temporary, part-time technician at the Gran Sasso lab (maximum 6 months). A $27.2 \%$ fringe benefit rate is included for all non-student salaries except the part-time technician, for whom the rate is $21.8 \%$.

Foreign travel continues to be a substantial portion of our operations costs. Our group is deeply involved in the commissioning of the MACRO detector in Italy. We have pursued all the various means to minimize our costs (APEX tickets, etc.). We have leased an apartment near the lab to minimize housing costs, yet this arnounts to $\$ 8,000 /$ year. In addition, a long term car lease (as opposed to weekly rentals) plus gasoline expenses amount to about $\$ 12,000 /$ year. Typical round trip plane tickets are $\$ 800-\$ 1000$ each, depending on season. At a minimum, our group makes 12 round trips per year. Hence, our foreign travel budget request is truly marginal and cannot be reduced without lessening our presence in Italy. The BU group until now has been one of the primary US groups involved with installation, debugging and turn-on of the MACRO detector for data.

In our supplies and services request, we have included the usual expenses for communications, supplies and publications. We continue to rely on the BU Electronics Design Facility for assistance in designing and prototyping electronics which BU must supply for the detector. In the coming year, this shop will be deeply involved with the final details of the fast wave form digitizer system for MACRO. The MACRO operating charges included in the budget arose from a decision made at a 1991 collaboration meeting by the U.S. institutional coordinators for the MACRO experiment. It was recognized that we will have a continuing need for supplies, tools, and test equiprnent at the Gran Sasso Lab. As an equitable method of supplying the needed equipment, it was decided that each U.S. group should be "taxed" $\$ 1000$ per year per person signing MACRO publications. This tax can be paid by providing needed equipment or by transferring cash to an account at the Gran Sasso Lab from which needed supplies can be purchased in Italy. For our group, the yearly contribution amounts to $\$ 8,000$. 
Our capital equipment budget represents BU's responsibility to provide all of the frontend electronics for MACRO's scintillator system. For the attico (now under construction) this includes the phototubes, bases, cables, signal fanouts, LED switchboxes and the entire laser/fiber optic calibration system. In addition, $\mathrm{BU}$ has taken on a major portion of the wave form digitizer (FWFD) system. We are currently leading the design effort for the FWFD and expect to be involved in the manufacture and implementation of the system. As in the past, these capital equipment funds are formally requested from DOE by Barry Barish. We include the anticipated $\mathrm{BU}$ equipment in this request to streamline the grant modification following DOE's action on Barish's request.

\section{Summary of Publications by the BU Particle Astrophysics Group}

A complete bibliography of our group members' publications for the past 5 years is presented below. During this period various members of our group have been involved in several experimental programs including the IMB Proton Decay experiment (Stone), E-816 Neutrino Oscillations experiment at BNL (Stone, Chrysicopoulou), the MACRO cxperiment at Gran Sasso (entire group), and the CDF experiment at Fermilab (Kearns). For the IMIB and MACRO experiments, several papers have been submitted to the International Cosmic Ray Conferences in Moscow, Adelaide, and Dublin. In these cases, the number of papers submitted is large due to the restrictions imposed by the conference organizers. Nevertheless we have listed all in the bibliography below. Currently, the MACRO Collaboration has performed additional work and is in the process of submitting scveral papers with physics results to refereed journals. Since these papers are not listed in our bibliography, but will be submitted during the evaluation of this grant proposal, we summarize here those papers and BU's involvement:

The MACRO Experiment at Gran Sasso: This technical paper, submitted to Nuclear In. struments and Methods in June 1992, details the hardware configuration of MACRO's first supermodule, which has been in operation since February 1989. Its purpose is to provide the primary reference for the apparatus used for physics results of MACRO's Phase I data runs. J. Stone is a primary author and chairman of the MACRO editorial board for this paper.

Miuon Astronomy with the MACRO Detector at Gran Sasso: This will be a long, detailed paper submitted to the Astrophysical Journal. Data collected during 1989 and 1090 with 
MACRO's first supermodule as well as 1990-1991 data with 2 SM will be presented. Analyses have been performed on MACRO's muon data which include: i) an all-sky search for a DC excess of muon events from any $3^{\circ} \times 3^{\circ}$ bin in the sky; ii) a search for a steady state muon flux from the direction of sources Cygnus X-3, Hercules $X-1$ and the Crab Nebula; iii) a search for periodic signals correlated with the pulsar and/or precessional periods of Cygnus X-3 and Hercules X-1; iv) a search for a short term ( $\sim$ day) enhancement of the muon flux from Cygnus $\mathrm{X}-3$ in correlation with reported radio outbursts of the source. No statistically significant signals have been observed in any of the searches, resulting in improved upper limits on anomalous muon fluxes at the depth of the MACRO detector. J. Stone and E. Kearns have contributed to the analyses of these data and J. Stone is on the MACRO editorial board responsible for producing the final manuscript.

\section{Mersurement of Decoherence Function with the MACRO Experiment at Gran Sasso:}

This paper, submitted to Nuclear Physics B in April 1992, presents a measurement of the underground muon decoherence function using multiple muon events. The data sample will be unambiguously reconstructed muon pairs giving 20,450 pairs from a run with the frst supermodule and 31,400 from data with two supermodules. A detector independent analysis will be presented for different zenith regions and rock depths, allowing for direct comparison with various models of cosmic ray interactions. The measured decoherence functions will be compared to the predictions of a Monte-Carlo simulation. R. Cormack is on the cditorial board responsible for producing the final manuscript.

Study of the Primary Cosmic Ray Composition: This paper, also submitted to Nuclear Physics $B$ in April 1992, presents a study of the rates of different multiplicities of muons in the MACRO detector. Data is fron: two runs: 1) the first MACRO data set with only 1 supermodule, 2) more recent data with 2 supermodules. The results of this study are compared to Monte. Carlo predictions for 2 composition models.

A Search for Nuclearites or Strange Quark Matter with the MACRO Detector: This paper, submitted to Physical Revieu Letters in April 1992, is based on work performed primarily by $G$. Liu of Caltech. Although designed as a monopole detector, MACRO is also sensitive to "nuclearites" or strange quark matter in cosmic rays. The combination of a three-month run in 1989 and a run from October 1989 to April 1991 has yielded a flux limit of $1.1 \times 10^{-11} \mathrm{~cm}^{-2} \mathrm{sr}^{-1} \mathrm{~s}^{-1}$ for nuclearites with mass $10^{-11} \mathrm{~g}<m<0.1 \mathrm{~g}$. For 
$m>0.1 \mathrm{~g}$, the limit is $1.1 \times 10^{-14} \mathrm{~cm}^{-2} \mathrm{sr}^{-1} \mathrm{~s}^{-1}$. The velocity range of nuclearites to which MACRO is sensitive extends down to near the escape velocity of the earth and therefore covers almost all of the possible velocity ranges of nuclearites.

\section{Bibliography}

\section{Refereed Journals}

1. "MACRO: A Large Area Detector at the Gran Sasso Laboratory," MACRO Collaboration, Nuovo Cimento $\underline{9 \mathrm{C}}$ (1986) 281.

2. "The MACRO Detector at the Gran Sasso Laboratory," MACRO Collaboration, Nucl. Inst. \& Meth. A264 (1988) 18.

3. "A Search for Neutrino Oscillations," P. Astier, J. L. Stone et al., Physics Lett. B220 (1989) 646.

4. "A Search for Neutrino Oscillations," P. Astier, J. L. Stone et al. Nucl. Phys. B335 (1990) 517.

5. A Study of Penetrating Cosmic Ray Muons and a Search for Large Scale Anisotropy at the Gran Sasso Laboratory," MACRO Collaboration, Phys. Lett. B249 (1990) 149.

6. -Simultaneous Observation of Extensive Air Showers and Underground Muons at the Gran Sasso Laboratory," MACRO Collaboration, Phys. Rev. D42 (1990) 1396.

7. "Arrival Time Distributions of Very High Energy Cosmic Ray Muons in MACrO," MACRO Collaboration; accepted for publication by Nucl. Phys. B, Sept. 1991.

8. -An Electromagnetic Calorimeter for the Small Angle Regions of the Collider Detector at Fermilab," G. Brandenburg, E. Kearns et al., Nucl. Inst. and Meth. A267 (1988) $25 \%$.

9. Transverse-Momentum Distributions of Charged Particles Produced in $\bar{p} p$ Interactions at $\sqrt{s}=630$ and $1800 \mathrm{GeV}$, F. Abe, E. Kearns et al., Phys. Rev. Lett. $\underline{61}$ (1988) 1819.

10. "Mensurement of the Inclusive Jet Cross Section in $\bar{p} p$ Collisions at $\sqrt{s}=1.8 \mathrm{TeV}$," F. Abe et al., Phys. Rev. Lett. 62 (1089) 613.

11. "Mleasurement of W-Boson Production in $1.8 \mathrm{TeV} \bar{p} p$ Collisions," F. Abe et al., Phys. Rev. Lett. 62 (1989) 1005.

12. "Limits on the Masses of Supersymmetric Particles from $1.8 \mathrm{TeV} \bar{p} p$ Collisions," F. Abe et al., Phys. Rev. Lett. 62 (1989) 1825. 
13. "Dijet Angular Distributions from $\bar{p} p$ Collisions at $\sqrt{s}=1.8 \mathrm{TeV}, " \mathrm{~F}$. Abe et al., Phys. Rev. Lett. 62 (1989) 3020.

14. "Measurement of the Mass and Width of the $Z^{0}$ Boson at the Fermilab Tevatron," F. Abe et al., Phys. Rev. Lett. $\underline{63}$ (1989) 720 .

15. "Search for Heavy Stable Charged Particles in $1.8 \mathrm{TeV} \bar{p} p$ Collisions at the Fermilab Collider," F. Abe et al., Phys. Rev. Lett. $\underline{63}$ (1989) 720.

16. "Search for the Top Quark in the Reaction $\bar{p} p \rightarrow$ Electron + Jets at $\sqrt{s}=1.8 \mathrm{TeV}$," F. Abe et al., Phys. Rev. Lett. 64 (1990) 142.

17. "Search for New Heavy Quarks in Electron-Muon Events at the Fermilab Tevatron Collider," F. Abe et al., Phys. Rev. Lett. $\underline{64}$, (1990) 147.

18. "Measurement of the Ratio $\sigma(W \rightarrow e \nu) / \sigma(Z \rightarrow e e)$ in $\bar{p} p$ Collisions at $1.8 \mathrm{TeV}$," $\mathrm{F}$. Abe et al., Phys. Rev. Lett. $\underline{64}$ (1990) 152 .

19. "Two-Jet Differential Cross Section in $\bar{p} p$ Collisions at $\sqrt{s}=1.8 \mathrm{TeV}$," F. Abe et al., Phys. Rev. Lett. $\underline{64}$ (1990) 157.

20. "Measurement of $D^{*}$ Production in Jets from $\bar{p} p$ Collisions at $\sqrt{s}=1.8 \mathrm{TeV}$," F. Abe et al., Phys. Rev. Lett. 64 (1990) 348.

21. " $K_{S}^{0}$ Production in $\bar{p} p$ Interactions at $\sqrt{s}=1.8 \mathrm{TeV}, " F$. Abe et a., Phys. Rev. D (Rap. Comm.), $\underline{40}$ (1989) 3791.

22. "Search for a Light Higgs Boson at the Tevatron Proton-Antiproton Collider," F. Abe ct al., Phys. Rev. D (Rap. Comm.), 11 (1990) 1717.

23. "Jet-fragmentation Properties in $\bar{p} p$ Collisions at $\sqrt{s}=1.8 \mathrm{TeV}, " F$. Abe et al., Phys. Rev. Lett. 65 (1990) 968.

24. "Measurement of the $W$-Boson Mass," F. Abe et al., Phys. Rev. Lett. 65 (1990) 2243.

25. "Two-jet Invariant Mass Distribution at $\sqrt{s}=1.8 \mathrm{TeV}, " \mathrm{~F}$. Abe et al., Phys. Rev. D 41 (1990) 1722.

26. "Pseudorapidity Distributions of Charged Particles Produced in $\bar{p} p$ interactions at $\sqrt{s}=630$ and $1800 \mathrm{GeV}, "$ F. Abe et al., Phys. Rev. D (Rap. Comm.), 41 (1990) 2330 .

27. "Top-quark Search in the Electron+Jets Channel in Proton-Antiproton Collisions at $\sqrt{s}=1.8 \mathrm{TeV}, "$ F. Abe et al., Phys. Rev. D $\underline{43}$ (1991) 664 . 
28. "Measurement of the $W$-Boson Mass in 1.8-TeV $\bar{p} p$ Collisions" F. Abe et al., Phys. Rev. D $\underline{43}$ (1991) 2070.

29. "Measurement of $\sigma B(W \rightarrow e \nu)$ and $\sigma B\left(Z \rightarrow e^{+} e^{-}\right)$at $\sqrt{s}=1800 \mathrm{GeV}, \mathrm{F}$. A be et al., Phys. Rev. D. 44 (1991) 29.

30. "Measurement of the $W P_{T}$ Distribution in $\bar{p} p$ Collisions at $\sqrt{s}=1.8 \mathrm{TeV}, " F$. Abe et aj., Phys. Rev. Lett. 66 (1991) 2951.

31. "Measurement of QCD Jet Broadening in $\bar{p} p$ Collisions at $\sqrt{s}=1.8 \mathrm{TeV}, " \mathrm{~F}$. Abe et al., Phys. Rev. D 44 (1991) 601.

32. "A Determination of $\sin ^{2} \theta_{W}$ from the Forward - Backward Asymmetry in $\bar{p} p \rightarrow$ $Z^{0} X \rightarrow e^{+} e^{-X}$ Interactions at $\sqrt{3}=1.8 \mathrm{TeV}, " F$. Abe et al., Phys. Rev. Lett. $\underline{67}$ (1991) 1502.

33. "Measurement of the $e^{+} e^{-}$Invariant Mass Distribution in $\bar{p} p$ Collisions at $\sqrt{s}=1.8$ TeV," F. Abe et al., Phys. Rev. Lett. $\underline{67}$ (1991) 2418.

34. "Search for $W I \rightarrow e \nu$ and $W I \rightarrow \mu \nu$ in $\bar{p} p$ Collisions at $\sqrt{s}=1.8 \mathrm{TeV}, "$ F. Abe et al., Phys. Rev. Lett. 67 (1991) 2609.

35. "Measurement of the $Z P_{T}$ Distribution in $\bar{p} p$ Collisions at $\sqrt{s}=1.8 \mathrm{TeV}, " \mathrm{~F}$. Abe et al., Phys. Rev. Lett. 67 (1991) 2937.

36. "Measurement of $B_{0} \bar{B}_{0}$ Mixing at the Fermilab Tevatron Collider," $F$. Abe et al., Phys. Rev. Lett. 67 (1991) 3351.

37. "Inclusive Jet Cross-Section in $\bar{p} p$ Collisions at $\sqrt{s}=1.8 \mathrm{TeV}, " F$. Abe et al., Phys. Rev. Lett. 68 (1992) 1104.

38. "The Topology of Three Jet Events in $\bar{p} p$ Collisions at $\sqrt{s}=1.8 \mathrm{TeV}, " \mathrm{~F}$. Abe et al., Phys. Rev. D $\underline{45}$ (1992) 1448.

39. "A Lower Limit on the Top Quark Mass from Events with Two Leptons in $\bar{p} p$ Collisions at $\sqrt{s}=1.8 \mathrm{TeV}, "$ F. Abe et al., Phys. Rev. Lett. $\underline{68}$ (1992) 447.

40. "A Search for New Gauge Bosons in $\not p p$ Collisions at $\sqrt{s}=1.8 \mathrm{TeV}, " F$. Abe et al., Phys. Rev. Lett. 68 (1992) 1463.

41. "Properties of Events with Large Total Transverse Energy Produced in $\bar{p} p$ Collisions at $\sqrt{s}=1.8 \mathrm{TeV}, " F$. A be et al., Phys. Rev. D 45 (1992) 2249.

42. "A Limit on the Top Quark Mass From $\bar{p} p$ Collisions at $\sqrt{s}=1.8 \mathrm{TeV}, " \mathrm{~F}$. Abe et al., Phys. Rev. D $4 \underline{5}$ (1992) 3921. 
43. "Measurement of the Isolated Prompt Photon Cross-Sections in $\bar{p} p$ Collisions at $\sqrt{s}=$

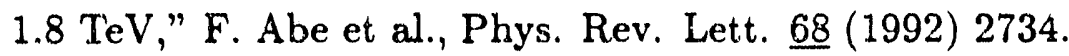

44. "Measurement of the Ratio $\sigma B(W \rightarrow r \nu) / \sigma B(W \rightarrow e \nu)$ in $\bar{p} p$ Collisions at $\sqrt{s}=1.8$ TeV," F. Abe et al., Phys. Rev. Lett. $\underline{68}$ (1992) 3398.

45. "A Measurement of the $B$ Meson and $B$ Quark Cross-Sections at $\sqrt{s}=1.8 \mathrm{TeV}$ Using the Exclusive Decay $B^{ \pm} \rightarrow J / \psi K^{ \pm}$," F. Abe et al., Phys. Rev. Lett. $\underline{68}$ (1992) 3403.

46. "Inclusive Jet Cross-Section in $\bar{p} p$ Collisions at $\sqrt{s}=1.8 \mathrm{TeV}$," F. Abe et al., FERMILAB-Pub-91-231; submitted to Phys. Rev. Lett.

47. "Lepton Asymmetry in $W$ Decays from $\bar{p} p$ Collisions at $\sqrt{s}=1.8 \mathrm{TeV}, " \mathrm{~F}$. Abe et al., FERMILAB-PUB-91-263-E; submitted to Phys. Rev. Lett.

48. "A Measurement of the Production and Muonic Decay Rate of $W$ and $Z$ Bosons in $\bar{p} p$ Collisions At $\sqrt{s}=1.8 \mathrm{TeV}, " F$. Abe et al., FERMILAB-PUB-92-69-E; submitted to Phys. Rev. Lett.

49. "A Problem in Rotation Therapy with X-Rays: Dose Distributions with an Axis of Symmetry," Cormack, A. M. and Cormack, Robert A., International Journal of Radiation Oncology, Biology and Physics, $\underline{13}, 1987$.

Publications in Conference Proceeding.

1. "Monopole and Gravitational Collapse Trigger Circuit for the MACRO Scintillator Sub-system," MACRO Collaboration, M. Calicchio ei al., Proceedings of the 20th International Cosmic Ray Conference, Moscow, $\underline{6}$ (1987) 334.

2. "The MACRO Detector for the Gran Sasso Laboratory," MACRO Collaboration, M. Calicchio et al., ibid., 500 .

3. "Design and Performance of the MACRO Liquid Scintillator Detector," ibid., 504.

4. "The Response of Streamer Tube to Highly Ionizing Particles," MACRO Collaboration, M. Calicchio et al., ibid., 507.

5. "The Streamer Tubes for the MACRO Experiment at the Gran Sasso Laboratory," MACRO Collaboration, M. Calicchio et al., ibid., 510.

6. "The Track-Etch Detector for the MACRO Experiment," MACRO Collaboration, M. Calicchio et al., ibid., 513.

7. "The MACRO Large Area Detector for the Gran Sasso Laboratory," MACRO Collaboration, M. Calicchio et al., Proceedings of Neutrino Masses and Neutrino Astrophysics, Ashland, 1987, 463. 
8. "Status Report of the MACRO Experiment at the Gran Sasso Laboratory," MACRO Collaboration, M. Calicchio et al., Proceedings of the International Workshop on Neutrino Telescopes, Venezia 1988, 139.

9. "Results From the First Run of the First Supermodule of the MACRO Detector at the Gran Sasso Laboratory," MACRO Collaboration, R. Bellotti et al., Workshop on Theoretical and Phenomenological Aspects of Underground Physics 1989, Editions Frontieres, 49.

10. "First Coincidences Between Extensive Air Showers and Underground Muons at the Gran Sasso Laboratory," MACRO and EASTOP Collaborations. R. Bellotti et al., M. Aglietta et al., ibid., 67.

11. "Simultaneous Observation of Extensive Air Shower and Underground Muons at the Gran Sasso Laboratory," MACRO and EASTOP Collaborations. R. Bellotti et al., M. Aglietta et al., paper presented at the 1989 EPS International Conference on High Energy Physics, Madrid; Nuclear Physics B (Proc. Suppl.) 16 (1990) 486.

12. "Status Report of the MACRO Experiment at Gran Sasso," MACRO Collaboration, M. Calicchio et al., Proceedings of the 20th International Workshop on Weak Interaction and Neutrinos, Ginosar (1980); Nuclear Physics B (Proc. Suppl.) 13 (1990) 368.

13. "Results from the First Run of the First Supermodule of the MACRO Detector at Gran Sasso Laboratory," MACRO Collaboration, R. Bellotti et al., paper presented at first TAUP Workshop, L'Aquila (1989).

14. "Study of the Primary Cosmic Ray at $E_{0}=10^{13}-10^{16} \mathrm{eV}$ by Simultaneous Obserration of Extensive Air Showers and Underground Muons at the Gran Sasso Laboratory," MACRO and EASTOP Collaborations, R. Bellotti et al., M. Aglietta et al., Procedings of the 21st International Cosmic Ray Conference, Adelaide, 9 (1990) 331.

15. "Simultaneous Observation of Extensive Air Showers and Underground Muons at the Gran Sasso Laboratory," MACRO and EASTOP Collaborations, R. Bellotti et al., M. Aglietta et al., ibid., 335.

16. "Multiple Muon Physics with the MACRO Detector at Gran Sasso," MACRO Collaboration, R. Bellotti et al., ibid., 356.

17. "Single Muon Physics with the MACRO Detector," MACRO Collaboration, R. Bellotti et al., ibid., 371. 
18. "A Search for Magnetic Monopoles with the MACRO Detector at Gran Sasso," MACRO Collaboration, R. Bellotti et al., ibid., 75.

19. "The Track-Etch Detector for the MACRO Experiment at the Gran Sasso Laboratory," MACRO Collaboration, R. Bellotti et al., ibid., 225.

20. "Overview of the MACRO Detector at the Gran Sasso Laboratory," MACRO Collaboration, R. Bellotti et al., ibid., 25.

21. "Search for Stellar Collapse with the MACRO Detector," MACRO Collaboration, R. Bellotti et al., ibid.

22. "MACRO at Gran Sasso: Results and Perspectives," MACRO Collaboration, R. Bellotti et al., Proceedings of the Second International Workshop on Neutrino Telescopes, Venezia 1990, 183.

23. "Combined Measurements of Deep Underground Muons and of the Electromagnetic Components of Extensive Air Showers," MACRO and EASTOP Collaborations, R. Bellotti et al., M. Aglietta et al., ibid., 209.

24. "First Results from the MACRO Experiment at the Gran Sasso Laboratory," MACRO Collaboration, R. Bellotti et al, invited paper at the Neutrino 90 Conference, CERN, Genera; Nuclear Physics B (Proc. Suppl.) 19 (1991) 128. Recent Invited Talks, Seminars, Colloquia of the BU Group

1. "Recent Results from the MACRO Experiment at Gran Sasso," J. L. Stone, seminar, University of Toronto, March 1991.

2. "Muon Astronomy with Underground Detectors," J. L. Stone, invited talk, Washington APS meeting, April 1991.

3. "Magnetic Monopole Search Experiments;" "Muon Astronomy: Fact or Fiction;" "Observation of Supernova SN1987a;" J. L. Stone, invited talks, Second School on Non-Accelerator Particle Astrophysics, International Center for Theoretical Physics, June 1991, Trieste, Italy.

4. "Muon Astronomy with the MACRO Experiment at Gran Sasso," J. L. Stone, invited talk. International Lepton Photon and High Energy Physics Conference, August 1991 , Geneva, Switzerland.

5. "Muon Astronomy with the MACRO Experiment at Gran Sasso," J. L. Stone, invited talk, Theoretical and Experimental Aspects of Underground Physics (TAUP91), September 1991, Toledo Spain. 


\title{
TASK D: NEUTRINO ASTROPHYSICS AND PROTON DECAY
}

\author{
Faculty: $\quad$ Professor L.R. Sulak (co-P.I.) \\ Associate Professor J. Stone \\ Research Associate Professor S.T. Dye (co-P.I.)
}

Graduate Students: F. Welte

\section{Introduction}

Boston University continues to play a leadership role in the IMB experiment. We have begun implementing the four-year plan outlined in our last proposal. This plan called for two new initiatives to succeed IMB, which terminated data-taking in April 1991. The efforts to actively participate in Super-Kamiokande and DUMAND have progressed well. We succerded in convincirig the IMB collaboration to join forces with the Japanese Kamioka group for a series of tests at KEK, and potentially for participation in Super-Kamiokande. Major steps were taken towards identification of hardware responsibilities for the beam tests and ratification of a collaboration agreernent. Stone was elected co-spokesman of the IAIB collaboration to provide vigorous leadership in the new endeavor. Our hardware contribution to DUMAND nears completion and preparations for data analysis have been finished. In parallel with the efforts on the new initiatives, good progress has been made analyzing IMIB-3 data. Within the last few months, four IMB manuscripts were submitted for journal publication. In addition to an instrumentation paper describing the IMB-3 detector in detail, these papers reported analyses of the contained data, the upward-going muon data, and the downward-going muon data.

In the following sections we describe in more detail the progress on the new initiatives . and on andyzing IMB data. We begin with an overview of the proposed accelerator-based calibration tests of a large water Cerenkov detector. Next, we outline the DUMAND fast time digitizer task. In conclusion, we review the latest physics results derived from analysis of INIB data. 


\section{Beam Tests at KEK}

The IMB collaboration's interest in obtaining DOE support for a series of experimental tests at KEK in conjunction with the Japanese Kamioka group was formally expressed in a letter of April 15, 1992 to Dr. Williarns. This letter was followed the next month by a proposal, which included detailed budget for equipment funds. The collaboration's hardware contributions were carefully negotiated during a series of meetings (Japan in February 1992, Philadelphia in April 1992, Boston in May 1992, und Spain in June 1992) with our Japanese colleagues. In addition to instrumenting the test tank with IMB light collectors, the collaboration proposed to provide the laser calibration and water purification systems for the beam test experiment. We at Boston took responsibility for the PMT readout electronics and mounting structures. DOE support for these tasks was requested as a supplement to our base operations in May 1992.

The technique of the KEK experiment will consist of directing beams of charged particles, $e^{-}, \mu^{-}$, and $\pi^{-}$of well defined momenta in the range of $100-2000 \mathrm{MeV} / \mathrm{c}$, and low energy neutrinos $\left(E_{\nu} \leq 2 \mathrm{GeV}\right)$, into a 1 kton water Cerenkov detector similar to Kamiokande-2 or IMB-3. The detector will be instrumented consecutively with 20" Hamamatsu phototubes with photocathode coverage equal to that of the Kamiokande-2 detector and later with 8 " phototubes and light collectors as in the IMB-3 detector. The readout and control electronics will be the same as used in each of these experiments.

The charged particle beams will allow us to collect signals in the detector from particles of well defined identity and momenta entering the detector with well known geometry. The particles will be put into the detector by means of a beam pipe penetrating the detector wall at 6 different locations. At each of these locations, the beam will be projected to 3 different depths into the detector volume. The analysis of the data collected in this way will allow us to: verify track reconstruction procedures; verify track identification methods; provide a precise energy calibration; verify the light tracking Monte Carlo simulation; and verify the muon decay detection efficiency.

The neutrino beam will allow us to collect a sample of neutrino interactions contained in the detector volume. This beam will have a well understood and small $\nu_{\mathrm{e}}$ component. The analysis of these data will allow us to: study neutrino interactions in water and the outgoing lepton momentum distribution; and study the final states of the neutrino interactions to provide better predictions of nucleon decay background from atmospheric 
neutrino interactions. A neutrino beam at KEK will open the possibility of performing a long baseline neutrino oscillation experiment in conjunction with Super-Kamiokande.

\section{DUMAND}

In June of 1989 Boston University joined the DUMAND-II project. This was before the project was approved or funded. Exploiting the technical expertise of Boston University in fast electronics, which was lacking in the DUMAND collaboration, the task of designing the fast time digitizing circuit was selected. This commitment helped the project secure approval and funding.

The fast time digitizer detects pulses from DUMAND optical modules and latches the pulse timing with nanosecond precision. After encoding each pulse with a channel number and diagnostics, the digital information is sent $30 \mathrm{~km}$ via optical fiber to the trigger station on shore. The heart of the fast digitizer is a gallium arsenide application specific integrated circuit. This chip is the design product of electronics engineers at Boston University. Other high energy physics projects are planning to employ this circuit, which demonstrates the utility and versatility of the design.

Now the digitizer task nears completion. The DUMAND array is scheduled for deployment and subsequent data-taking in the summer of 1993. Boston University plans to reap scientific reward for this significant hardware contribution by analyzing DUMAND data.

\section{IMB-3 Data Analysis}

More than 2000 neutrino interactions, spanning neutrino energies from $20 \mathrm{MeV}$ to $\sim 1 T \mathrm{~T}$. were recorded by the $I M B$ detector. Two classes of events are represented by these interactions. Lower energy events $(<2 \mathrm{GeV})$ are typically fully contained in the detcctor volurne, while higher energy events enter from below and travel upward. Both . clastes are due to atmospheric neutrinos.

Contained events from a $7.7 \mathrm{kt}-\mathrm{yr}$ exposure of IMB-3 are used to measure the atmospheric neutrino compasition and to estimate proton partial lifetimes. When combined with the $4.3 \mathrm{kt}$.yr exposure from IMB-1 and IMB-2, sensitivity to proton decay into $e^{+} \pi^{\circ}$ and other low-background modes nearly reach the level of $10^{33}$ years. For lower-energy modes and those with greater background, the sensitivity is a factor of three to ten less. Data from IMB-1 and 2, plus our preliminary analysis of IMR-3 date, give no compe!ling 
evidence that nucleon decay has been observed above background. The atmospheric neutrino composition measurement uses only IMB-3 data. We find an anomalously low fraction of "muon-like" events in the total sample of single ring events. This finding agrees with an independent measurement of the fraction of events with a muon decay signal. Although oscillation of neutrino flavor is a possible explanation of the data, further studies are required before this conclusion can be reached.

Over 600 upward-going muon events recorded over the entire working life of the IMB detector are used to search for muon neutrino oscillations. Two methods are employed: comparing the measured rate with the expected rate; and the ratio of upward-going muons which stop in the detector to those which exit is used to search for deviations from the expected energy spectrum. This latter technique is free of flux and cross-section normalization uncertainties. No evidence for $\nu_{\mu} \rightarrow \nu_{r}$ oscillation is found. $90 \%$ c.l. limits on $\delta m^{2}$ are derived in the range from 1 to $2 \times 10^{-1} \mathrm{eV}^{2}$ for $\sin ^{2} 2 \theta>0.5$.

\section{Nucleon Decay}

Our preliminary lifetime limit for the decay mode $p \rightarrow e^{+} \pi^{\circ}$ is $8.5 \times 10^{32} \mathrm{yr}$. Back: ground limitation for this important process is not evident in our data. We believe we could have exceeded $10^{33}$ years with several more years of exposure. The prospect of probing lifetimes of $10^{34}$ years using Super-Kamiokande is enticing to any physicist interested in making a major contribution in this field.

We have in progress a search for proton decay modes favored by Grand Unified Theories. The lower part of the nucleon lifetime range predicted by these theories is testable. In SLSY SU(5) the decay rates of $p \rightarrow \nu K^{+}$and $n \rightarrow \nu K^{\circ}$ are $\sim 10$ times greater than other allowed 2-body decay moder. With the enhanced detector sensitivity associated with the installation of the $8^{n}$ PMT's (MBB-3) we study channels such as $p \rightarrow \nu h^{+}$with high efficiency $(\sim 13 \%)$. Our present limit is $5.0 \times 10^{31} \mathrm{yr}$ for $\mathrm{K}^{+} \rightarrow \pi^{\circ} \pi^{+}$. This search relies on reconstruction of the $\pi^{\circ}$ and/or the $K^{+}$mass. We show a scatter plot of invariant mass versus residual momentum for scanned Monte Carlo events in fig. D.1. The SUSY GLT derived from string theory $S U(5) \times U(1)$ favors the 2-body decay modes $p \rightarrow e^{+} \pi^{\circ}$, $p \rightarrow \mu^{+} \pi^{0}, p \rightarrow \nu \pi^{+}, n \rightarrow e^{+} \pi^{-}, n \rightarrow \mu^{+} \pi^{-}$, and $n \rightarrow \nu \pi^{\circ}$. The search for the eight rnodes mentioned probes theories which predict nucleon decay.

Beyond specific predictions, a search for all possible decay modes of proton and neu-

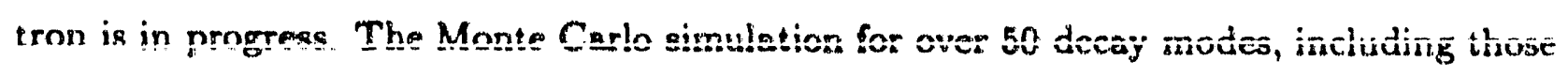


listed above, is complete. Limits for these modes will improve with the application of invariant mass and residual momentum analysis.

\section{Measurements of the Atmospheric Neutrino Flux}

Nearly isotropic fluxes of $\nu_{e}$ and $\nu_{\mu}$ are produced over a broad range of energies by cosmic-ray interactions with the atmosphere. Because of uncertainties in the primary cosmic-ray flux and models of hadron interactions with air, the absolute numbers of $\nu_{e}$ and $\nu_{\mu}$ can be predicted only to within $\pm 20 \%$. The ratio of the two flavors, which is independent of flux, is predicted with more certainty $( \pm 5 \%)$. Interactions of atmospheric neutrinos are recorded in underground detectors and constitute the dominant background to the search for nucleon decay. A majority of the interactions result from charged current quasi-elastic neutrino-nucleon scattering, producing a single charged muon or electron. The $\nu_{\mu}$ content of the atmospheric neutrino flux is thus approximated by the fraction of events containing a muon decay signal. A smaller than expected fraction was observed in the earlier IMB-1 experiment. Applying particle identification techniques to selected single particle events permits an independent, more reliable estimate of the flavor content of the atmospheric neutrino flux. Simulations of neutrino interactions at the relevant energies indicate strong correlations between $\nu_{e}$-induced $\left(\nu_{\mu}\right.$-induced) events and "electron-like" or "showrring" ("muon-like" or "non-showering") particles. A smaller than expected ratio of non-showering events to the total has been reported previously by Kamiokande and IMB-3. Similar discrepancies, found in the final data set of the IMB-3 experiment, are reported here.

For this study, a $3.3 \mathrm{kt}$ fiducial mass was used as target for atmospheric neutrinos. Detector triggers resulting from prompt firing of 70-900 PMTs were analyzed separately by two subgroups using independently developed calibrations and event filters. The 70 PMIT lower limit corresponds to roughly $50 \mathrm{MeV}$ of visible energy. A sample of events originating inside the fiducial volume was constructed from the results of both analyses. The combined efficiency for recovering contained events, assuming the filtering inefficiencies were independent, was $93 \%$. A total of 935 events within the $N_{P M}$ t cut were identified in data collected between May 1986 and March 1991, corresponding to a total exposure of $7.7 \mathrm{kt}-\mathrm{yr}$. Each event was scanned visually to determine the vertex, and the number and direction of Cerenkov rings present. This procedure identified 610 events with a single ring and 325 with multiple rings. By searching for coincidences annong PMTs fring after 
the trigger, muon decay electrons were detected with greater than $80 \%$ efficiency and contamination of only $2 \%$. In the single-ring (multiple-ring) sample, 208 (137) events were accompanied by at least one muon decay signal.

The physics capabilities of a large water Cerenkov detector are greatly extended through particle identification. A reliable means of discriminating between particle types is provided by measuring the geometry and intensity distribution of Cerenkov nit patterns. Diffuse, showering patterns are associated with $e^{ \pm}$and $\gamma$, while sharper, non showering patterns are associated with $\mu^{ \pm}, \pi^{ \pm}$, and $p$. Only single-ring events allow reliable determination of the parent neutrino flavor. Using three independent, automated identification algorithms, the single-ring events are classified as showering or non-showering. In most cases, the final classification is determined by a majority vote of the three tests: each test casts one vote. Because one test fails to classify some events, the identity of $26 \%$ of data events is determined by only two tests. In this subsample, the voting is unanimous for $73 \%$ of events. When the votes are split ( $7 \%$ of all single-ring events), the classification is made by the "more certain" result, based on quality factors calculated by the algorithms. In the single-ring sample, 378 events are identified as showering and 232 as non-showering.

To predict the showering/non-showering composition and the muon decay content of the sample, a simulation of neutrino interactions in water is combined with a threedimensional model of atmospheric neutrino production (including effects of muon polarization). Simulated interactions are then propagated through the detector Monte Carlo program and the data filtering and classification routines. Identical analysis has been performed on smaller sets of simulated data produced by two independent neutrino interaction/detector simulation packages. The performance of the identification algorithrns dors not depend on the particular neutrino physics model or detector response model which is uscd. Studies indicate the correlation between $\nu_{e}$-induced $\left(\nu_{\mu}\right.$-induced) events and identifird single-ring showering (non-showering) events is $87 \pm 1$ (stat) $\%(92 \pm 1$ (stat)\%).

A summary of the 851 day INB-3 contained data, and the simulated atmospheric neutrino data, is presented in Table D.1. Simulations using other atmospheric neutrino production modrls yield event rates up to $30 \%$ higher than the prediction presented here. Ratios of various classes of events predicted by the other models are, however, indistinguishable from those presented here. Several inconsistencies in the data are noted. The percentages of events with a muon decay and with a single Cerenkov ring are lower in 
the data than in the simulation. In the data, $37 \pm 2($ stat $) \%$ of all events are accompanied by a muon decay, while the simulation predicts $44 \pm 1$ (stat)\%. This inconsistency is more pronounced in the subsample of single-ring events, for which the fraction events with a muon decay signal is $0.34 \pm 0.02$ (stat), while a fraction of $0.43 \pm 0.01$ (stat) is predicted. In addition, the fraction of single-ring events in the data is $0.65 \pm 0.01$ (stat) compared with $0.72 \pm 0.01$ (stat) in the simulation.

The visible-energy spectra of all events and muon-decay events are compared with the simulation in fig. D.2. The shapes of the spectra agree. The discrepancy between the measured and predicted spectra above $1500 \mathrm{MeV}$ is limited to multiple-ring events, originating primarily from poorly understood multiple-pion production processes, and should not affect this study. Fig. D.3 demonstrates the agreement between the observed and expected spectra for single-ring events.

Discussion is now restricted to single-ring events. Momentum distributions for showcring, and non-showering events, along with their respective muon decay subsamples, are shown in fig. D.4, and fig. D.5. The shapes of the simulated spectra are well matched by the recorded data. Moreover, for both showering and non-showering events, the expected and observed muon decay fractions agree. However, the observed ratio of non-showering events to all single-ring events is lower than predicted. To further explore this inconsistency we focus our attention on the momentum ranges $100<p_{e}<1500 \mathrm{MeV} / \mathrm{c}$ and $300<p_{\mu}<1500 \mathrm{MeV} / \mathrm{c}$; low-momentum events are excluded to increase the reliability of particle identification, and high-momentum events are cut to veto exiting particles. In this range, non-showering events comprise $36 \pm 2($ stat $) \pm 2($ syst $) \%$ of the total. Based on the simulation, $51 \pm 1$ (stat) \pm 5 (syst)\% is expected. No time-dependent systematic drift of the non-showering fraction is observed in the data. When statistical and systematic uncertainties are combined, the measured fraction of non-showering single-ring events within the monentum cuts is $2.6 \sigma$ below expectation. The observed fraction of events with muon decays, independent of particle identification algorithms, is $4.0 \sigma_{\text {ata }}$ below expectation. Due to the uncertainty in the absolute flux, it is diffecult to determine if these discrepancies represent a deficit of non-showering events, an excess of showering events, or a combination of the two.

Atmospheric neutrinos interacting within the IMB-3 detector traverse distances of a few kilometers to $13000 \mathrm{~km}$ from their points of origin in the atmosphere. This long baseline 
translates to a sensitive window of $1 \mathrm{~km} / \mathrm{GeV}<\mathrm{L} / \mathrm{E}<10^{5} \mathrm{~km} / \mathrm{GeV}$ for possible oscillation of neutrino flavor. Neutrino oscillations are a possible, though not necessary, explanation of the data. The overall spectra agree reasonably well with predictions (figs. D.2 and D.3). The unexpected low fractions of non-showering events and events with a muon decay seem independent of zenith angle (see figs. D.6a and D.6b) and momentum (see figs. D.7a and D.7b). Much of the range of $\mathrm{L} / \mathrm{E}$ greater than $1 \mathrm{~km} / \mathrm{GeV}$ is sampled by measurements of externally produced upward-going muons from higher energy neutrinos. Within errors, no oscillation effect is observed in those data.

At this point, systernatic effects dominate the experimental errors in the measurement of the non-showering event fraction. Experiments designed to reduce these systematic uncertainties could uncover the cause of the apparent $\nu_{\mu}$ deficit (and/or $\nu_{e}$ excess). More precise measurements of muon and pion growth curves at high-altitude would improve confidence in the neutrino production model. Cross-section measurements using a controlled neutrino beam on oxygen targets, or a calculation more realistic than the Fermi Gas Model but amenable to event simulation, would help to eliminate uncertainties in the neutrino intcraction model. Exposing a test detector similar to IMB-3 to muons and electrons of appropriate cnergy from an accelerator would test the accuracy of particle identification.

In conclusion, the flavor composition of atmospheric neutrinos has been measured using two independent techniques: muon decays and particle identification. A deficit of muon neutrinos, or an excess of electron neutrinos, compared with prediction has been obscrved. The hamiolia experiment has observed a remarkably similar effect. Experiments and/or throretical work to reduce systematic uncertainties are possible and could discover the cause of the apparent discrepancy. 


\section{Operations Budget Discussion}

We have requested funds for general electronics shop and machine shop costs and miscellaneous supplies to cover work not explicitly anticipated in the equipment budget below. Our original operations request did not include support for our participation in the KEK beam test.

Travel by our group to Japan in the coming year is an essential item. These trips are required for a number of reasons associated with the formation of the new JapaneseAmerican collaboration at KEK. These include engineering design sessions for the KEK tank and PMT mounting system, planning and positioning of the detector and counting house at KEK, collaboration meetings, proposal writing for SuperKamiokande, etc. In addition, we note that James Stone of our group has been selected by the IMB collaboration to serve as co-spokesman (with Henry Sobel, UCI) for the newly formed collaboration with the Japanese Kamioka group. In this capacity, he will need to travel to Japan for organization meetings, proposal presentations at KEK, and collaboration meetings. Every effort will be made to maximize the work accomplished on each trip and to minimize costs by using KEK housing and other cost saving techniques as we learn them.

Domestic travel is also important during this period, since it has become necessary for the IMB group to meet more frequently to organize and coordinate our efforts for the KEK beam test. Travel to foreign and domestic conferences and DUMAIND Collaboration mertings is also anticipated.

\section{Equipment Budget Discussion}

Equipment funds requested will enable the Boston University IMB group to fulfill its responsibilities in fabricating and installing our water Cherenkov detector in time for the beam test at $\mathrm{KEK}$ in the fall of 1993. By the end of the summer, we must fabricate and install the readout electronics, the photomultipliers with associated bases, cables, $\mathrm{HV}$, paddle cards, and the mechanical mounting and light barriers in the tank at KEK. We anticipate that installation of the equipment will require four six-week trips to Japan by the B.C. personnel responsible for assembling the components of the detector. Their presence at KEK will be critical to assembly and installation of the detector. The remainder of the equipment funds requested will provide support for engineering, technical, and shipping charges. 


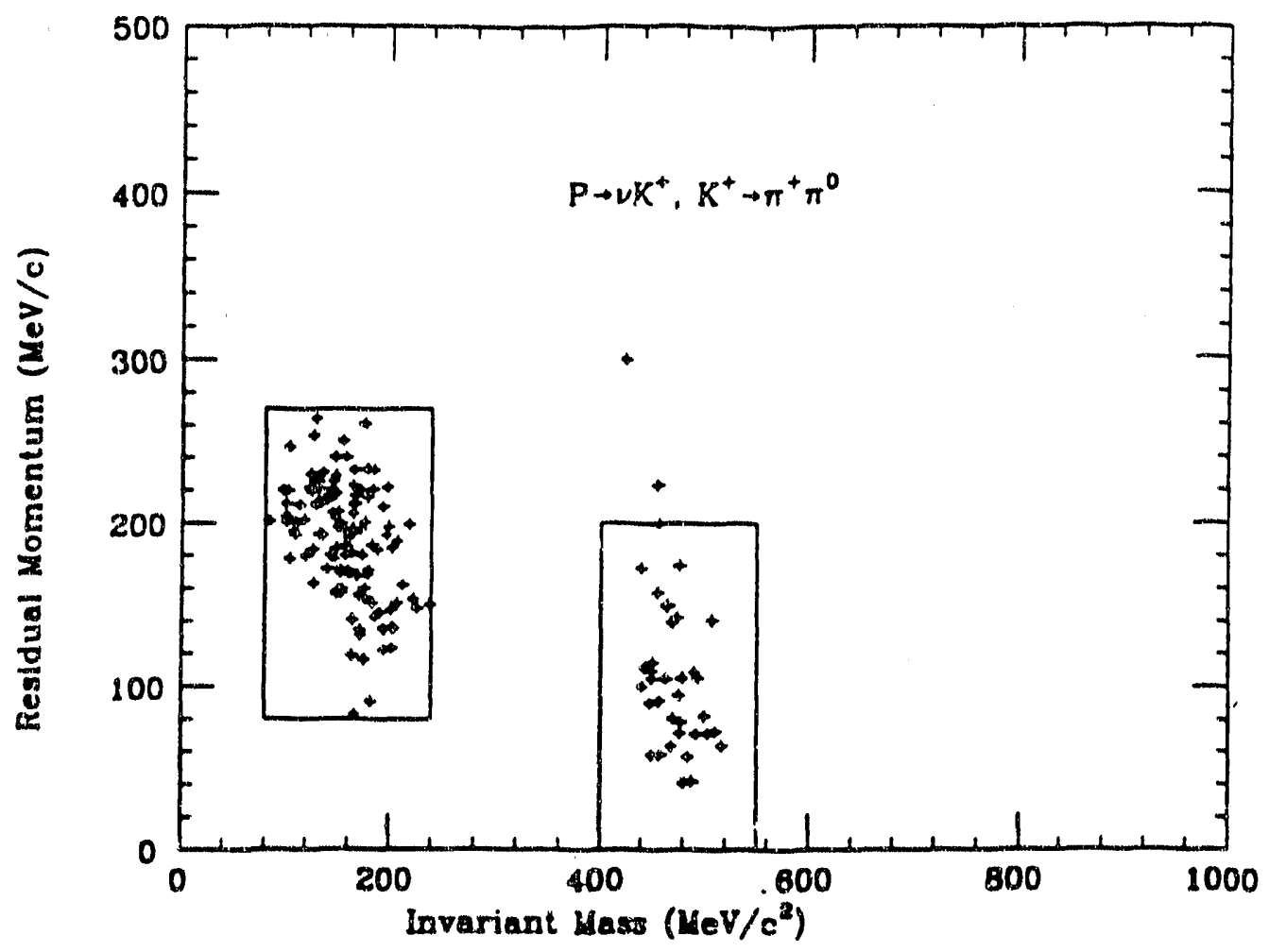

Figure D.1 Reconstruction of scanned Monte Cario $p \rightarrow \nu K^{+}, K^{+} \rightarrow x^{3} x^{+}$events.

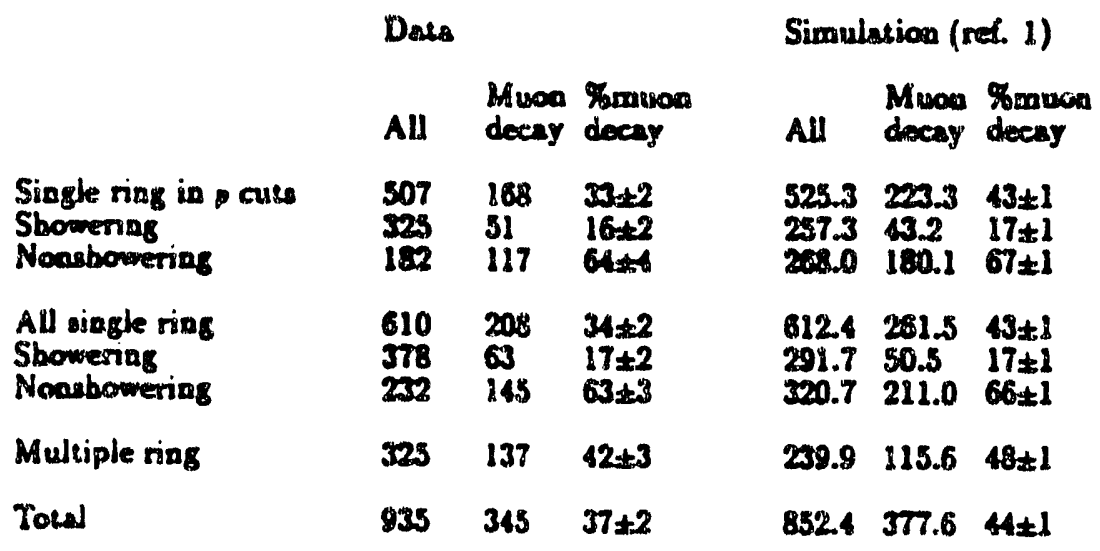

Table D.1 Summary of the 851 day IMB-3 contained data, and the simulated atmo. sphexic neutrino data. 


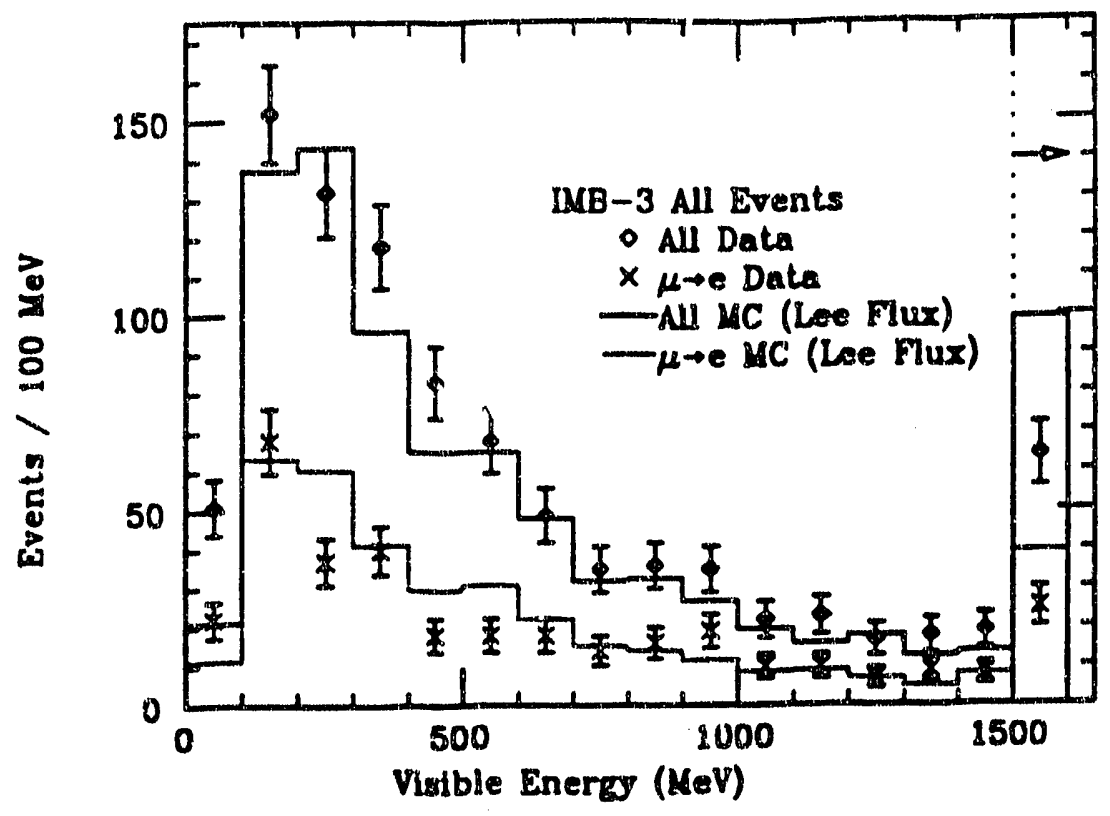

Figure D.2 Visible Energy of all contained events and those with a muon decay.

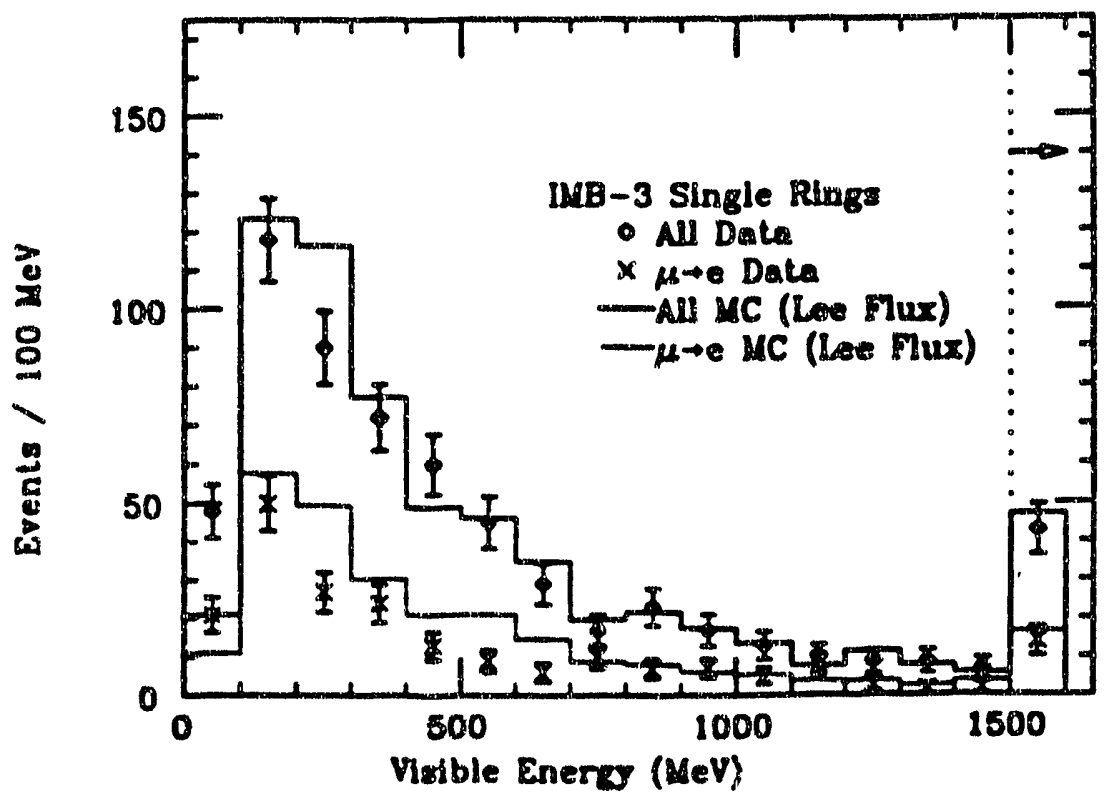

Figure D.3 Visible energy of all single-ring events and those with a muon decay. 


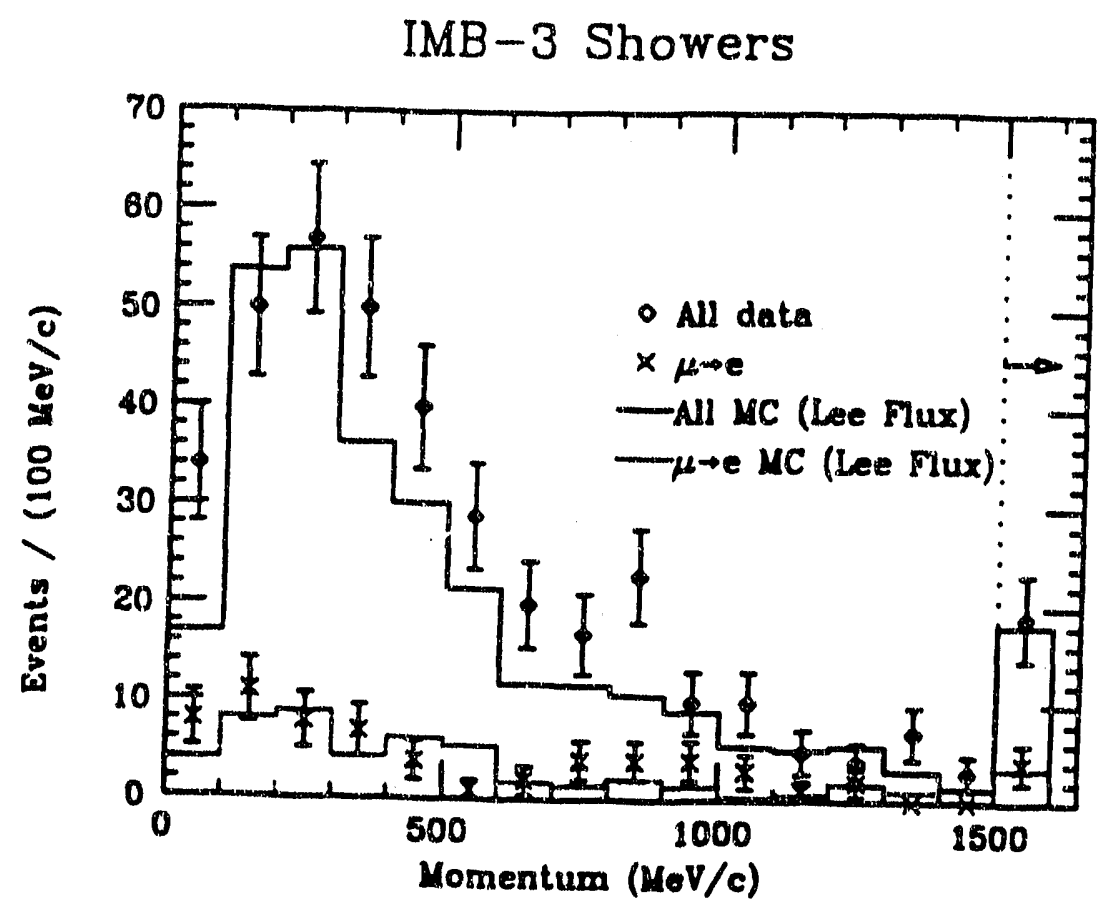

Figure D.4 Momentum of single-ring showering events and those with a muon decay.

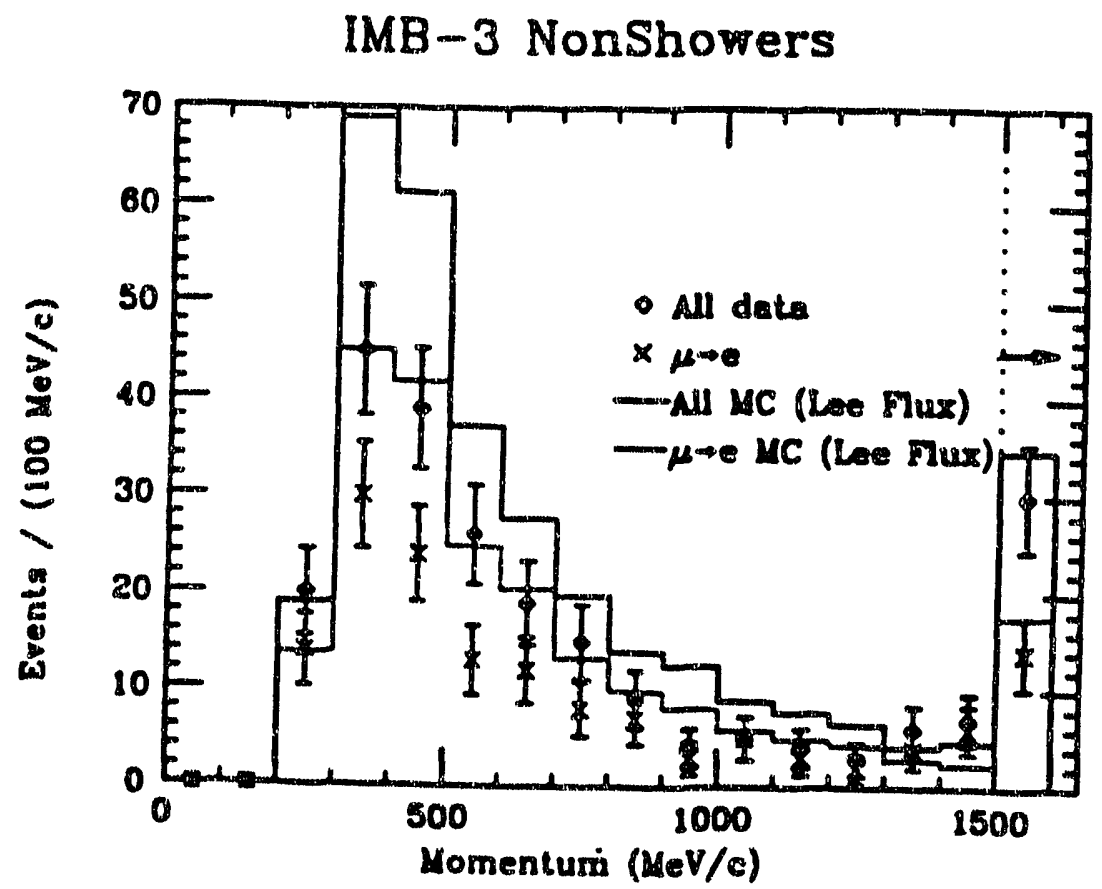

Figure D.5 Mornentum of single-ring non-showering events and those with a muon decay. 


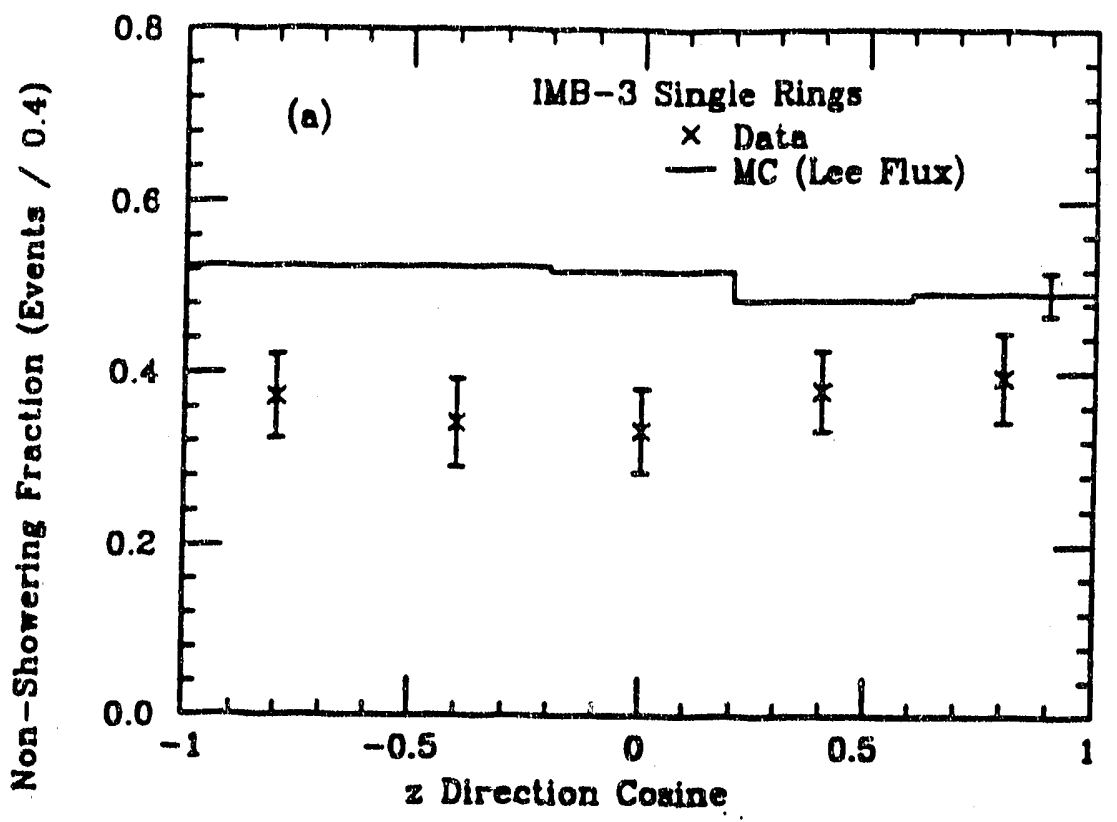

Figure D.6a Non-showering fraction vs. zenith angle.

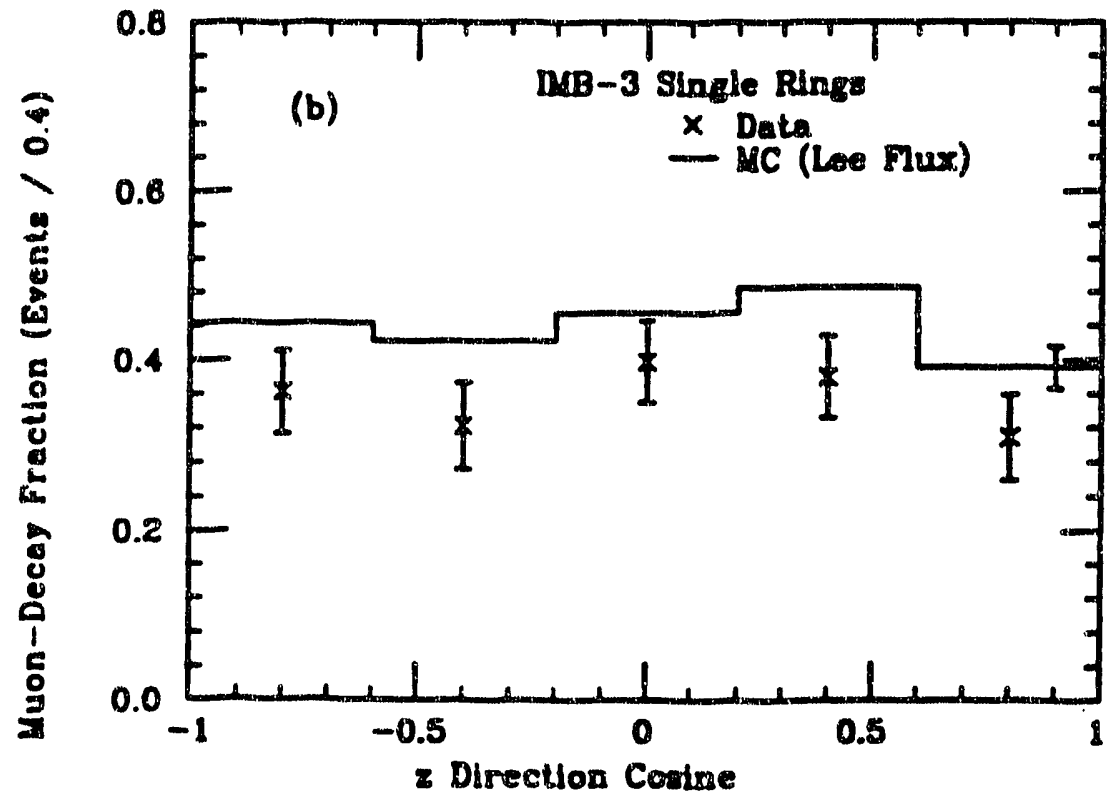

Figure D.6a Muon decay fraction vs. zenith angle. 


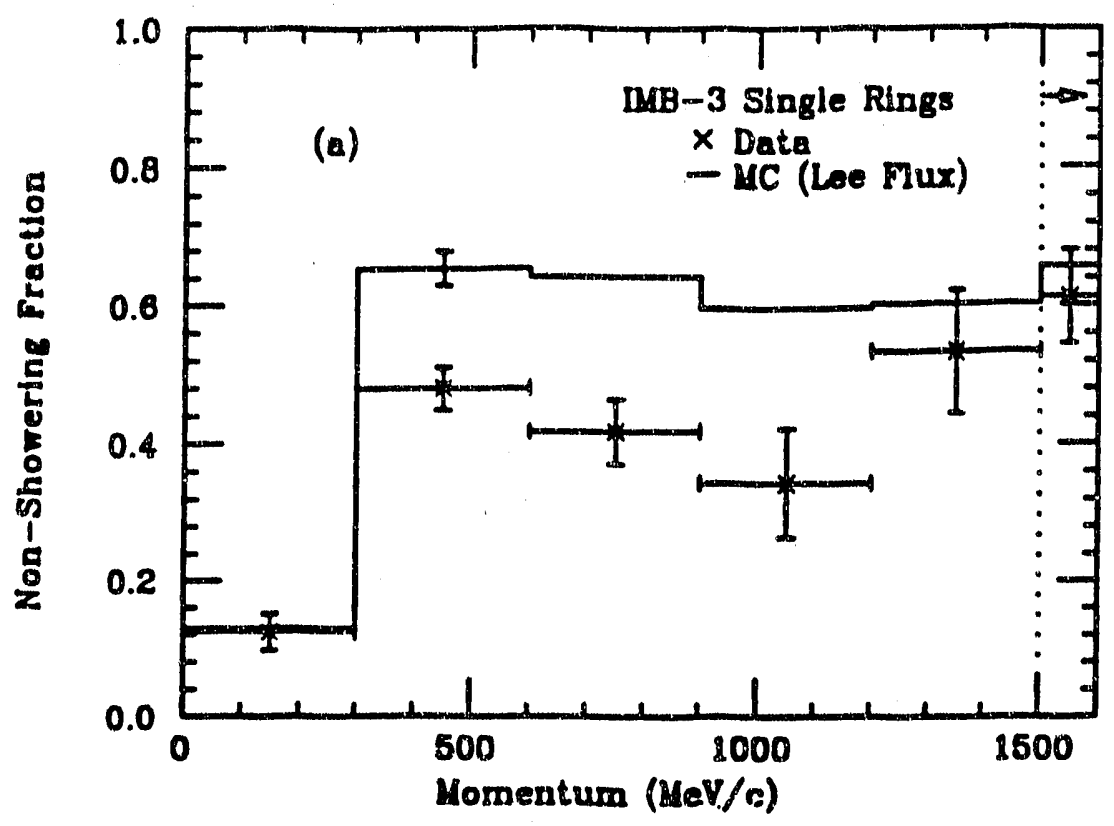

Figure D.7a Non-showering fraction v8. momentum.

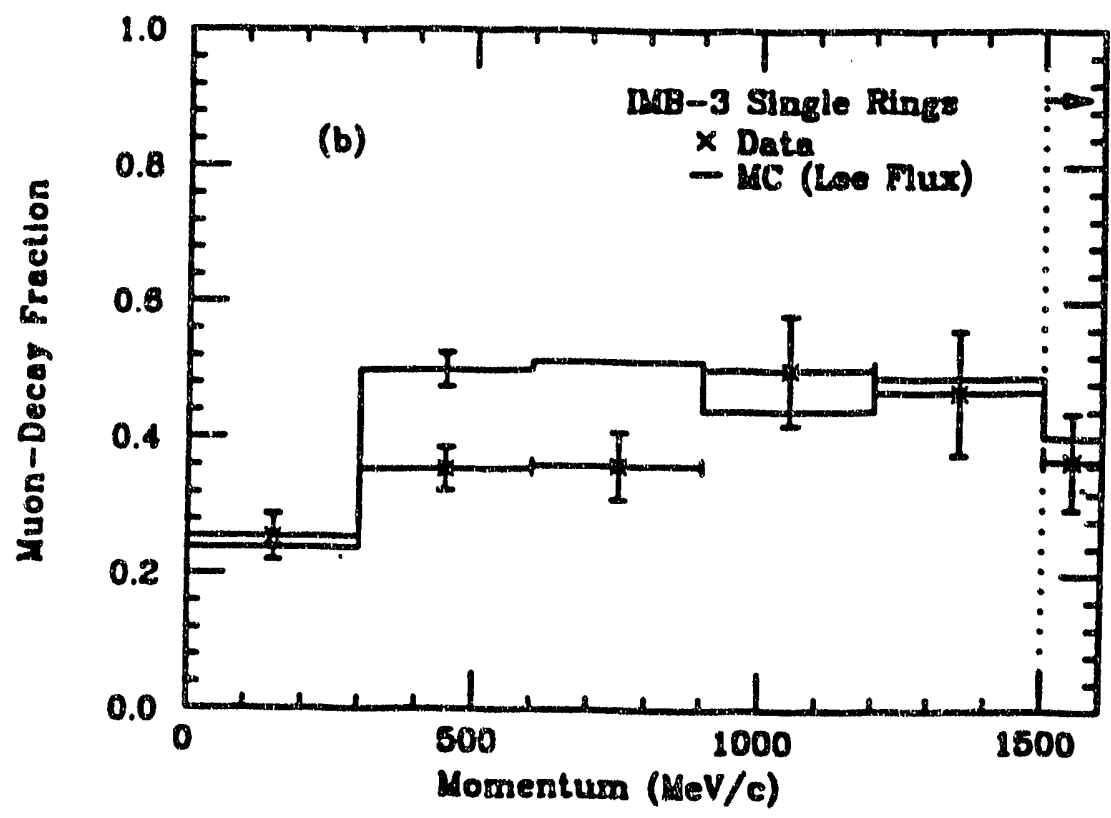

Figure D.7b Muon decay fraction vs. momentum. 
Recent Publications of Project Neutrino Astrophysics and Proton Decay 1991-1992

\section{A. Papers Published in Refereed Journals}

1. R. Becker-Szendy et i.., "The IMB-3 Detector," Boston University preprint BUHEP92-6, submitted to Nucl. Inst. and Meth., June 1992.

2. R. Becker-Szendy et al., "Study of Underground Muons during the January 1991 Radio Flare of Cygnus X-3," University of Hawaii preprint UH-511-745-92, submitted to Phys. Rev. Lett., March 1992.

3. R. Becker-Szendy et al., "A Search for Muon Neutrino Oscillations with the IMB Detector," Louisiana State University preprint LSU-HEPA-3-92, submitted to Phys. Rev. Lett., March 1992.

4. R. Becker-Szendy et al., "The Electron- and Muon-Neutrino Content of the Atmospheric Flux," Boston University preprint BUHEP-91-24, submitted to Phys. Rev. D, March 1992.

5. D. Casper et al., "Measurement of Atmospheric Neutrino Composition with IMB-3," Phys. Rev. Lett. 66, 2561-2564 (1991).

\section{B. Papers in Conference Proceedings}

1. R. Becker-Szendy et al., "The Electron and Muon Neutrino Content of the Atmospheric Flux," Proceedings of the Workshop on Long-Baseline Neutrino Oscillations, Fermilab, November 17-20, 1991, ed. M. Goodman (Batavia, Illinois: Fermi National Accelerator Laboratory, 1992). p. 159-170.

2. R. Becker-Szendy et al., "A Search for Muon Neutrino Oscillations with the IMB Detector," ibid., p. 177-180.

3. R. Becker-Szendy et al., "A Long Baseline Neutrino Oscillation Experiment Using the IMB Water Cerenkov Detector," ibid., p. 233-242.

4. R. Becker-Szendy et al., "A Search for Cygnus X-3 in Underground Muons During the 1090 Radio Outburst Using the IMIB Detector," Proceedings of the 22nd International Cosmic Ray Conference, Dublin. Ireland, 11-23 August 1991 (Dublin: Dublin Institute for Adranced Studies 1991), Vol. 4, HE 4.3.12, p. 615.

5. R. Becker-Szendy et al., "A Search for Muon Neutrino Oscillations with the IMB Detector," ibid., HE 5.1.2, p. 619. 
6. V. J. Stenger for the DUMAND Collaboration, "Proposed Very Long Baseline Experiment to Search for Neutrino Oscillations with DUMAND II," ibid., HE 5.3.6, p. 670 .

7. R. J. Wilkes for the DUMAND Collaboration, "Acoustical Positioning System for DUMAND II," ibid., p. 666.

8. V. Z. Peterson for the DUMAND Collaboration, "Ocean Technology Employed by the DUMAND Project," Proceedings of Oceans 91, 1-3 October 1991, Honolulu, Hawaii.

9. R. J. Wilkes for the DUMAND Collaboration, "DUMAND II Progress Report," Proceedings of Interactions between Particle and Nuclear Physics, May 1991, Tucson, Arizona.

\section{Reports}

1. A. Okiada et al., "Sensitivity of DUMAND II to AGN Neutrinos," ICRR-Report-26992-7, University of Tokyo, June 1992.

2. R. Bernstein et al., "Conceptual Design Report: Neutrino Physics after the Main Injector Upgrade," Fermilab, Batavia, Illinois, 1991 (unpublished).

3. S.T. Dye and E. Hazen, "Computer Simulation of the Strawman Digitizer for the DCMAND Experiment," DUMAND Internal Report No. DIR-1-91, January 1991. 


\section{TASK E: THEORETICAL PARTICLE PHYSICS}

Faculty:

Research Faculty:

Visiting Faculty:

Research Associates:

Graduate Students:
Professor K. Lane

Professor C. Rebbi

Associate Professor S. Y. Pi

Assistant Professor R. S. Chivukula

Assistant Professor A. Cohen

Assistant Professor R. Rohm

Professor R. Brower

Professor S. L. Glashow, Professor S. Nussinóv, Professor A. De Rújula

P. Fendley, M. Golden, S. Selipsky, Y. Shen, M. Dugan

G. Amelino-Camelia, B. Balaji, N. Berdenis, M. Camperi, W. Chen, D. Kominis, V. Koulovassilopoulos, P. Mavromatis, S. Myint, V. Pitsis, M. V. Ramana, R. Strilka

\section{RESEARCH PROGRAM}

In the following we review our research over the past several years and describe our goals for the coming year. This work may be broadly classified into the areas of electrowrak and fiavor symm try breaking, hadron collider phenomenology, cosmology and ast rophysics, mathematical physics and nonperturbative studies of quantum field theories. IIr cmphasize that these divisions are somewhat artificial because there are many points of contact and overlap, such is the signatures for technicolor at had $: n$ colliders, weak-scale baryognnesis, lattice computations of higher-order terms in the QCD chiral Lagrangian, and $w)(n$.

\section{A. Electroweak and Flavor Symmetry Breaking}

The origin of electroweak and flavor symmetry breakdown is a major cornponent of our research program. Much of this work has long been focused on the dynamical scenario 
for these symmetry breakings -... technicolor and extended technicolor (TC and ETC). Other approaches on which we have worked are the composite technicolor standard model, and the standard-model Higgs boson. We have played a major part in recent studies of precisely-measured electroweak quantities. Our research also includes investigations of the weak interactions of hadrons.

\section{A.1 Technicolor and Extended Technicolor}

Shortly after Weinberg and Susskind invented the technicolor mechanism of dynamical electroweak symmetry breaking [1], Lane and Eichten and Dimopoulos and Susskind [2] proposed enlarging the TC gauge group to allow for the generation of quark and lepton masses as well as masses for the technipions $\left(\pi_{T}\right)$ that typically occur in these extended technicolor models. In Ref. 2, Eichten and Lane pointed out that generic ETC theories have flavor-changing neutral currents among quarks and leptons that require an ETCboson mass scale $M_{E T C}=O(500 \mathrm{TeV})$ for their suppression. This large scale makes it very difficult to obtain values for the ETC-generated quark and lepton masses, $m \simeq$

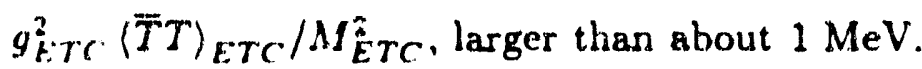

The most studied proposal to realize an acceptable ETC theory without large flavorchanging neutral currents is known as "walking technicolor" [3]. This approach has been discusued extensively in our earlier proposals. Separate works by Cohen and Lane addresued the issue of the generality of the ladder-approximation result that the anomalous dimension of the technifermion bilinear, $\gamma_{m}$, equals one at the chiral breaking scale, $I_{T C} \simeq 250 \mathrm{GeV}[4,5]$.

The standard walking technicolor scenario seems unable to account for the very large mass of the top quark. To overcome this problem, several authors have proposed that ETC interactions act in concert with TC to drive electroweak symmetry breaking, even though a large hierarchy exists between the $\Lambda_{T C}$ and $M_{E T C}[6]$. In Ref. 6 it was argued thas. if the TC co upling runs fairly slowly and the ETC coupling strength $g_{E T C}^{2} / 4 \pi$ is very close to a particular large, "critical" value, $\langle\bar{T} T\rangle_{E T C}$ may be greatly enlarged relative cven to its cuhancement in pure walking technicolor, $\langle\bar{T} T\rangle_{E T C} \sim\left(M_{E T C} / \Lambda_{T C}\right)^{2}\langle\overline{T T}\rangle_{T C}$, while $\langle\bar{T} T\rangle_{T C}$ itself is little changed. This means that it is possible to achieve $m_{1} \sim$ $g_{E T C}^{2}\langle\bar{T} T\rangle_{E T C} / M_{E T C}^{2} Z 100 \mathrm{GeV}$ while the scale of electroweak symmetry breaking is not raised above $250 \mathrm{GeV}$. 
Chivukula, Cohen and Lane (CCL) showed in Ref. 7 that the technifermion condensate, properly renormalized at $M_{E T C}$, is not enhanced as suggested in [6]. If ETC may be consistently represented below $M_{E T C}$ by a local four-fermion interaction, the renormalization of $\bar{T} T$ between $\Lambda_{T C}$ and $M_{E T C}$ is controlled entirely by $T C$ interactions, with $\gamma_{m} \leq 1$. Then, $\langle\bar{T} T\rangle_{E T C} \sim\left(M_{E T C} / \Lambda_{T C}\right)\langle\bar{T} T\rangle_{T C}$ at most. Nevertheless, the enhancement of $m_{t}$ envisaged in Ref. 6 may still occur.

By treating the ETC interaction at energies below $M_{E T C}$ as a Nambu - Jona-Lasinio interaction, CCL showed that the $M_{E T C}$-to- $\Lambda_{T C}$ hierarchy occurs only if the technifermion chiral symmetry breaking phase transition is second order and $g_{E T C}^{2} / 4 \pi$ is fine-tuned to be very close to the critical value required for chiral symmetry breaking. In this case, the effective ETC interaction below $M_{E T C}$ reduces to a linear sigma model which has light scalar bosons in addition to the massless Goldstone bosons of spontaneous symmetry breaking. In the limit of weak TC interactions, these scalars may be thought of as $\bar{T} T$ states tightly bound by the ETC interactions. They have mass $\sim(2-10) \Lambda_{T C}$, anomalously light Conpared to the ETC scale. Regardless of the symmetry structure of the ETC interaction, one of these iight scalars, $\phi$, is an electrically neutral state, reminiscent of the Higgs boson of the standard model. The effective description of ET $C$ below METC is then a nonlocal intrraction involving the exchange of this light scalar. Furthermore, this scalar has Yukawa couplings $l_{j}$, to technifermions and ordinary fermions which are unsuppressed by powers of $M_{E T C}$. In the spontaneous symmetry breaking, $\phi$ develops a vacuum expectation value $\langle 0\rangle \simeq A_{T C}$ and - depending on the size of $Y_{f}$ - technifermions and the top quark may acquire unsuppressed hard masses $m_{f} \simeq Y_{f}\langle\phi\rangle$.

Lane and T. Appelquist are preparing an arti le for Reviews of Modern Physics on dynamical electroweak and flavor symmetry breaking. This review will serve two purposes: It will be the first comprehensive review of this subject in almost ten years. In this tirne, there has been considerable development of new approaches to this subject, including walking technicolor, electroweak symmetry breaking driven in part by strong extended technicolor, and studies of the effect of technicolor degrees of freedom on presisely measured clectroweak quantities. There have also been several new approaches to ETC model building. Second, this review is intended to stimulate new ideas for resolving old problems. Tw wo prime exanples are: 
- The time is ripe for a re-examination of the strong-CP problem in theories with dynamical electroweik symmetry breaking [8]. A number of ideas that have been proposed in the context of "standard" Higgs models - a massless up-quark, an invisible axion, to name two - have never been realized in the dynamical scenario. An interesting suggestion of Holdom and Peskin that the growth of the QCD coupling above the TC scale could raise the axion mass seems tailor-made for walking technicolor because of the many colored technifermions that may occur (see, e.g., section A.2 below). But, then, why don't quarks also condense above the TC scale? Finally, if CP-violation is spontaneous, driven by vacuum alignment [8], is there any way around the problem of having too much energy stored in the walls between different CP-domains? This question has been taken up recently by Preshill, et al., and by Krauss and Rey [9]. If there is no way around the domain-wall problem, there remains the possibility of explicit CP-violation ETC interactions. It is unknown whether this possibility can be phenomenologically acceptable.

- There is still no very satisfactory picture of how the top quark can be so heavy (and ncutrinos so light) in ETC theories. This, of course, is the nub of the flavor problem and is what strong extended technicolor is intended to solve. Yet, there is no attractive model incorporating the heavy top-quark. Recent model-building proposials by Sundrum and by Randall [10] sidestep the whole flavor issue by putting all flavor breaking at an unspecified high scale, well above $M_{E T C}$. These proposals deserve caseful consideration. for they amount to giving up on trying to solve the flavor problem at accessible energies.

Cohen will continue research into strong-ETC theories. An important part of the question involves understanding the behavior of physical quantities as the strength of the ETC coupling is adjusted relative to the scale of ETC gauge symmetry breaking. The question is atin to asking how QCD would behave if the gluons had a mass, $M_{\mathcal{G}}$. For $M_{\mathcal{C}} \ll$ $\Lambda_{Q C D}$, the theory would look much the sarne as the real world; in particular, $\alpha_{Q C D}\left(\Lambda_{Q C D}\right)$ would be large and quarks confined. For $M_{C}>\Lambda_{Q C D}$, the strong interactions would look like the weak interactions: infrased slavery would disappear, the coupling would remain weat at all cnergy scales, and so on. The interesting question is how to connect these two extremes, and what happens as the two scales become very close. General constraints äse piovicied by complementarity and anomajy-matching conditions, but the important 
questions are dynamical ones. In particular, what are the values of physical parameters like the decay constant for technipions and the techniquark constituent masses?

\section{A.2 Symmetry Breaking Phenomenology}

If technicolor underlies electroweak symmetry breaking, spin-one technihadrons, $\rho_{T}$, exist and decay into pairs of technipions, $\pi_{T}$ - including, but not necessarily limited to, the longitudinal components of the $W^{ \pm}$and $Z^{0}$ bosons themselves [11]. These $\rho_{T}$ will be produced at hadron colliders with rates depending on their color-SU(3) quantum numbers: $\sigma\left(\rho_{T}\right)=O\left(\alpha^{2}\right)$ if they are color-singlets, $\sigma\left(\rho_{T}\right)=O\left(\alpha_{S}^{2}\right)$ if they are color-octets. In principle, they can be discovered at the SSC, and it may even turn out that some of them are accessible at the Tevatron.

The phenomenology of walking technicolor (and a fortiori strong extended technicolor) is expected to be very different from that described in Ref. 11. The principal difference comes from the fact that the enhancement of $\langle\bar{T} T\rangle_{E T C}$ raises the masses of technipions from former, small estimates and may even make them so heavy that some or all of the decay channels $\rho_{T} \rightarrow \pi_{T} \pi_{T}$ are closed.

Following the work of Lane and Eichten [12], Lane and his student, M. V. Ramana, undert(x)k an extensive study of walking technicolor phenomenology in an explicit class of multiscalc models [13]. These models had three species of technifermions: One weak doublet $\psi=\left(\psi^{\prime}\left(U^{\prime}, D\right)\right.$ transforming as the antisymmetric tensor of the technicolor group, $S U\left({ }^{\circ} T C\right)$ and as a singlets under the color group $S U\left(3_{C}\right)$ and a flavor group $S U\left(N_{F}\right)$; three doublets $Q=(U, D)$ of techniquarks transforming as $\left(\mathbf{N}_{\mathrm{TC}}, \mathbf{3}_{\mathbf{C}}, \mathbf{1}_{\mathbf{F}}\right)$ under these three groups: and $N_{F}$ doublets $L=(N, E)$ of technileptons transforming as $\left(N_{\text {TC }}, 1_{C}, N_{F}\right)$. The i condensed near the electroweak scale and, to study the consequences of a hierarchical multiscale rnodel, $Q$ and $L$ were forced to condense at a considerably lower scale. The condition $\gamma_{m}=1[4]$ and the $O\left(a_{T C}^{2}\right)$ results of Ref. 5 were essential for the determination of chiral symmetry breaking scales and masses of $\rho_{T}$ and $\pi_{T}$. A third feature that was incorporated into this study was that of large weak isospin breaking. This leads to neutral $\rho_{r}$ and $\pi_{T}$ that are ideally-mixed states, i.e., color-octets $\rho_{\bar{D} D}$ and $\rho_{\bar{U} U}, \pi_{\bar{D} D}$ and $\pi_{\bar{U} U}$; color-triplets $\pi_{\bar{N} U}$ and $\pi_{\bar{E}}$; and so on.

Larse and Ramana concentrated on the neutral color-octet $\rho_{T}$, strongly produced in parton-parton collisions. Because of $\pi r$ mass enhancemerits and il leal mixing, these appear

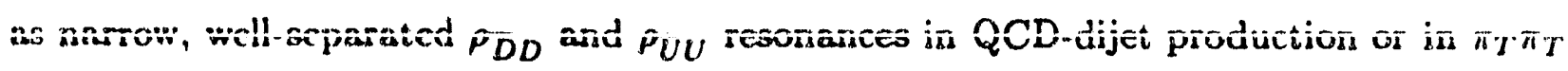


production with a limited number of final states. Two generic phenomenologies were found: (A) $\rho_{\bar{D} D}$ with mass $200-250 \mathrm{GeV}$ decaying exclusively to dijets and $\rho_{\bar{U} U}$ in the range $350-55 \%$ GeV decaying to a few $\pi_{T} \pi_{T}$ combinations; (B) $\rho_{\bar{D} D}$ with mass $375-425 \mathrm{GeV}$ and $\rho_{\bar{U}}$ in the range $500-700 \mathrm{GeV}$, both decaying to a few $\pi_{T} \pi_{T}$ modes. The $\rho_{\bar{D} D} \rightarrow$ dijet signal of case $A$ is large at all colliders and is being sought now at the Tevatron. The $\pi_{T} \pi_{T}$ production rates in both cases are of $O(10) \mathrm{pb}$ at the Tevatron and $O(10) \mathrm{nb}$ at the SSC. The technipions can be sought in the next high luminosity run of the Tevatron and may be excludable if backgrounds are not too severe. Experiments at the SSC certainly should be able to determine whether they exist.

In the coming year, Lane's student, B. Balaji, will study the hadron collider signal rates for the color-singlet sector of these multiscale walking techricolor models. It is necessary first to compute the production and decay rates for the color-singlet $\rho_{T} \rightarrow \pi_{T} \pi_{T}$ processes at the parton-model level. If these rates look promising, Balaji and Lane will carry out detailed PYTHIA-based simulations of signals and backgrounds to assess more accurately the observability of these phenomena at the SSC.

Finally, if the electroweak symmetry breaking sector contains colored particles weighing a fow hundred $\mathrm{GeV}$, they will be copiously produced at a hadron supercollider. Colored technipions can rescatter into pairs of gauge bosons. As proposed by Bagger, Dawson, and Valencia [14], this leads to gauge boson pair rates far larger than in the standard model. Chivubula. Golden and Ramana reconsidered this mechanism in Ref. 15, and illustrated it in a model in which the rates can be reliably calculated. They showed that the observation of both un enhanced rate of gauge-boson-pair events and colored particles would be a signal that the colored particles were pseudo-Goldstone bosons of symmetry breaking.

\section{A.9 Phenomenology of a $Q C D$-like Theory with Many diduors}

In a recent paper, Cahn and Suzuki argued that a relatively light, $O(500) \mathrm{GeV}$, Higgslike particle exists as a bound state pole in the scattering of technipions [16]. Motivated by this. Chivutula and $G$, olden investigated the occurrence of flavor-singlet scalar resonances in Goldstone boson s attering in the presence of soft explicit symmetry breaking [17]. Using the $O(n)$ model in the large $n$ limit as an example, they showed that there are two alternatives to the Cahn-Suzuki possibility: First, the "Higgs" can be a "virtual bound state" pole on the second complex-s sheet. In this case, the $\pi_{r} \pi_{r}$ scattering amplitudes

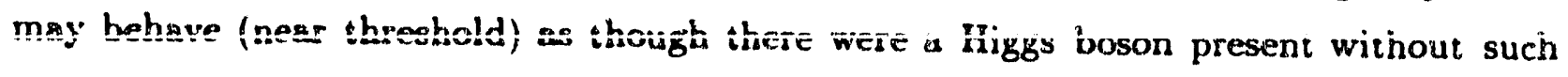


a particle actually existing. Second, the particle predicted by Cahn and Suzuki may be unbound, decaying to $\pi_{T} \pi_{T}$.

Expanding on these ideas, Chivukula and Golden considered the behavior of longitudinal $W$ and $Z$ scattering in the $O(n)$ model [18]. It is generally assumed that elastic $W$ and $Z$ scattering exhibits either resonances below $\sim 1 \mathrm{TeV}$ or enhancements indicating the presence of new strong interactions at or above $1 \mathrm{TeV}$. They showed that there is another possibility: The electroweak symmetry breaking sector may be strongly coupled at low energies because of the presence of large numbers of particles - such as technipions other than the longitudinal components of the $W$ and $Z$. In this case the elastic $W$ and $Z$ scattering amplitudes may be small without the appearance of any discernible resonances, while the total scattering cross sections are large. In such a model, the number of $W W$ and $Z Z$ events never is large: discovering the electroweak symmetry breaking sector depends on the observation of the other particles and the ability to associate them with symmetry breaking. In this sense, the symmetry breaking sector in such a model is "hidden".

Chivukula, Golden, Korninis, and Ramana [19] have calculated the production rate of gauge-boson pairs at the SSC in a model with a "hidden" electroweak symmetry breaking sector. They showed that the $W W$ and $Z Z$ scattering signal of electroweak symmetry breaking is suamped by the background. They also calculated the gluon fusion contribution to $2 Z$ production in such a model, and showed that even this contribution is smaller than the background. They conclude, therefore, that we cannot necessarily rely on gauge boson pairs as a signal of the dynamics of symmetry breaking.

With Dugan. Chivukula and Golden [20] investigated the dynamics of a QCD-like gauge therry with more than two or three flavors. Generalizing an argument of Sundrum and Soldate [21], they showed that the chiral expansion in a theory with $N$ flavors breaks down at or below $4 \pi f / \sqrt{N}$. Here, $f$ is the Goldstone boson decay constant. They argued that the breakdown of the chiral expansion is associated with the appearance of physical states other than Goldstone bosons, leading to the surprising conclusion that there must be resonances in a theory with six or eight flavors which are much lighter than one would expect by asalogy to QCD.

\section{A.4 Higgs Bosars Phenomenology}

Selipsky, Dimopoulos, Lynn and Tetradis investigated the possibility of bags in the: Higgs condensate [22]. in which fermions decrease their energy by binding to a self- 
generated region of smaller vacuum expectation value. Such soliton configurations are interesting as modifications to free-particle states in strongly coupled theories - including the standard model. Deep bags would have observable effects on particle masses, couplings, and decay modes. Using classical equations of motion, they obtained numerical solutions which in appropriate limits reproduced the SLAC bag applied to a quark-Higgs system. Quantum corrections were shown to be important for deep bags. Consistency and completeness of the standard model helps limit the range of possibilities.

Chivukula and his student, V. Koulovassilopoulos, are investigating the phenomenology of a composite Higgs model at the SSC. When the parameters of the model are adjusted to produce a "Higgs boson" of mass greater than about $600 \mathrm{GeV}$, the effects of the physics responsible for binding the boson start to become significant. They are now estimating the size of these effects.

Chivukula and another student, D. Kominis, have considered the constraints of triviality on general two-Higgs models. They have shown that, just as in the one-Higgs case, the scalar masses in such a theory are typically constrained to be less than approximately $700 \mathrm{Gel}$. They are now investigating the phenomenology of these acceptable models.

\section{A.5 Precision Tests of the Electroueak Interactions}

Finding evidence of new physics without sufficient energy to produce it directly is possible if one can carefully analyze the quantum fluctuations which arise from physics at all scales. Starting with his earliest work on the delay of unitarity in $W W$ scattering due to neu physics above $\sim 1 \mathrm{TeV}$ [23]. Selipsky has been concerned with developing a systematic organization of such radiative corrections, to allow identification of different and/or common effects in a variety of settings. With Lynn, Stuart and Levinthal, he has bern constructing a framework for separating out and grouping the gauge structures occurring in electroweak radiative corrections, using the nonabelian properties of gauge vertices to reorder them into irreducible gauge generator sequences [24]. Gauge-invariant "generalized self-energy functions" which include the universal parts thus isolated can then be uritten down and used in effective matrix elements. This work has been incorporated into data analysis programs used by the ALEPH Collaboration [25].

Golden and Randall recently studied the radiative corrections technicolor theories may make on precisely-measured electroweak quantities [26]. Even when technicolor respects 
the custodial $S U(2)$ symmetry, which guarantees no shift in the low-energy $\rho$-parameter, there may be large wave-function renormalizations of the $W$ and $Z$, and these can shift the mass of the $W$ or other parameters. They showed that the corrections to $M_{W}$ induced by the large number of technipions occurring in the one-family TC nodel may be discernible with higher-statistics measurements that can be made at the Tevatron collider.

In light of the fact ( $c f$. A.3) that the spectrum of a QCD-lil.. ' heory with many flavors is likely to be much different than might be expected from $O C$, Chivukula, Dugan, and Golden [27] have recently re-examined estimates of radiative corrections to electroweak parameters in technicolor theories. These corrections are evaluated by one of two techniques: either one estimates spectral function integrals using scaled QCD data [28], or one uises naive dimensional analysis with a chiral Lagrangian [26, 29]. The former yields corrections to electroweak parameters proportional to the number of flavors and the number of colors, while the latter is proportional to the number of flavors squared and is independent of the number of colors. Chivukula, Dugan and Golden explained this apparent contradiction and argued that, in a theory with more than two or three techniflavors, the technicolor resonances are likely to be much lighter than naively supposed. If this is the case, the radiative corrections to electroweak parameters due to technicolor may by much larger than naively estimated.

Chivukula, Selipsky, and E. H. Simmons (Harvard) have probed the possibility of nonoblique clectroweak radiative corrections appearing in currently feasible observations [30]. In extended technicolor theories, fermion masses are generated by ETC boson exchange with technifermions that develop condensates. In addition to effective mass operators, the same ETC bosons exchanged in other channels generate flavor-dependent corrections to couplings to $Z$ and $W$ gauge bosons, with effects proportional to the fermion mass. In particular, the top quark mass operator must also (by electroweak symmetry) give a correction to the $Z \bar{b} b$ coupling, which turns out to be proportional to ( $m_{t} / 3 \mathrm{TeV}$ ) instead of the standard model quadratic dependence. Assuming a minimal theory in which the ETC bosons have no weak charge, the correction is calculable up to a group-dependent factor, and leads to an $m_{t}$-dependent shift in the $Z \bar{b} b$ width of seven times the standard model value. This should be detectable in LEP data currently being obtained. 


\section{A.6 Weak Interactions of Hadrons}

Cohen studied the question of nonanalytic corrections to chiral perturbation theory and applied his results to radiative kaon and eta decays for which there had been a longstanding discrepancy between theory and experiment [31]. Given a low energy effective Lagrangian describing the interactions of Goldstone (or pseudo-Goldstone) bosons, chiral perturbation theory is a simple technique for constructing Green functions as a power series expansion in powers of momenta (or symmetry breaking masses) over a scale which is typically $4 \pi f_{\pi}$. There are well-known corrections to this analytic expansion, known as chiral logarithms. These nonanalytic terms are determined by unitarity, and are most easily computed by extracting the singular parts of Feynman diagrams constructed from the analytic expansion described above. In a chiral-perturbative expansion, these logarithms are small corrections to the leading terms. Cohen noted that there are circumstances where the nonanalytic terms are poles rather than logarithms, and these pole terms can be larger than the leading analytic parts. The contribution of this pole term may be computed for processes where it is dominant, and yields predictions for certain amplitudes in terms of other measured quantities. An example is $K_{L} \rightarrow \pi^{0} \gamma \gamma$, which may be related to $K_{L} \rightarrow \pi^{0} \pi^{+} \pi^{-}$. Other examples of processes that may be related in this way are $K_{S} \rightarrow \gamma \gamma$. and $\eta \rightarrow \pi^{0} \gamma \gamma$. These relations are well-satisfied by experiments. Investigation of similar relations in technicolor is being considered.

Golclen has also been interested in the static effective field theory of a heavy quark. This is a method for the inclusion of states involving a heavy quark (such as the $b$ ) in processes at low energy. For example, in a heavy.light meson, the heavy quark acts as A static color source. not reacting much to the cloud of light particles around it. This is the basis for an expansion in powers of the inverse of the heavy quark mass. Golden and B. Hill earlier constructed the full order $\alpha_{S}$ matching of the weak decay operators at order 1/m [32]. Golden has now completed work with M. Dugan and B. Grinstein showing how the heavy quark effective theory may be derived rigorously from $\mathrm{QCD}$, and discussing the structure of the Hilbert space of the effective theory [33]. 


\section{B. Hadron Collider Phenomenology}

It is very likely that the high energy and luminosity of the SSC will be needed to discover and study the physics of electroweak and flavor symmetry breaking. We know that the scale of electroweak symmetry breaking lies near $1 \mathrm{TeV}$, within reach of the SSC. We do not know the scale of flavor symmetry breaking. But several popular ideas related to or explaining flavor physics - standard or nonstandard (possibly supersymmetric) Higgs bosons, extended technicolor, and some scenarios for quark/lepton substructure - are also within the SSC's range. Several works described in the previous section dealt with the search for electroweak and flavor phenomena at the Tevatron and the SSC (see Refs. 16-19, 21-24). Here we describe our recent involvement in more detailed studies of this physics at hadron colliders.

Lane and Chivukula are very actively involved in hadron collider studies, as are several of their local collaborators, including and Golden and E. H. Simmons (Harvard). Lane is a co-leader of the GEM Detector Collaboration's Physics Group (with Frank Paige) and has spent much of the past year helping prepare the GEM Expression of Interest (EOI) [34] ka Letter of Intent (LOI) [35]. In 1992, he has been working on the preparation of the GEMI Technical Design Report (TDR). These recent activities will be described further below. Chirukula is the organizer of a workshop on SSC physics to be held at the Aspen Center for Physics in the summer of 1993.

\section{B.1 Recent Research}

Chivukula has investigated the experimental consequences of quark and lepton compositeness at the SSC [36]. As discussed by Eichten, Lane and Peskin, substructure will be manifested at energies below the scale of compositeness, $\Lambda$, by a set of four-fermion contact interactions arising from the exchange of constitueast particles [37]. The dominant effect of quark substructure at the SSC would be an enthancement in the inclusive jet spectrum at high transverse momentum. If both quarks and leptons are composite, there would be an excess of events at large dilepton invariant mass in the Drell-Yan process.

If deviations from standard model expectations are observed, it will be important to determine the Lorentz structure of the four-fermion operators, since this may provide the only clue about the strong interactions responsible for binding the quarks and leptons. 
Chivukula and Randall showed that it is possible to use the front-back asymmetry of highmass Drell-Yan events collected in one standard SSC year $\left(\int \mathcal{L} d t=10^{40} \mathrm{~cm}^{-2}\right)$ to constrain the form of the contact interactions if the quark/lepton substructure scale $\Lambda \lesssim 15 \mathrm{TeV}$ [38].

Chivukula, Golden and Simmons recently examined four- and six-jet events at the LHC and SSC as a potential signal for new physics. These signals could arise, e.g., from the strong production of new color-octet scalars (which decay to two jets each) and from color-sextet fermions (decaying to three jets each). After considering the QCD backgrounds and the effects of a generic SSC detector's jet-energy resolution, they concluded that it should be possible to detect the strongly-produced fermions with masses up to $1700 \mathrm{GeV}$ and the scalars up to $600 \mathrm{GeV}$ in one standard year at the SSC $[39,40]$.

Lane and F. Paige organized the the preparation of the Physics Chapter of the GEM LOI (together with Bing Zhou of Boston University and James Branson of U. C. San Diego) [35]. This chapter responded to a set of five questions posed by the SSC Program Advisory Committee (PAC) regarding GEM's physics capabilities. These questions concerned GEM's ability to discover the standard Higgs boson in the mass range 80 $800 \mathrm{GeV}$. a $250 \mathrm{GeV}$ top quark, and missing-transverse-energy signals of a $400 \mathrm{GeV}$ quark and a $300 \mathrm{GeV}^{\prime}$ gluino; GEM's jet energy resolution for the $Z^{0}$ and a $1 \mathrm{TeV} Z^{\prime 0}$; and the question of the complementarity of the GEM design and physics goals to those of the SDC detertor. Lane et al. organized a group of about 20 people to carry out the simulations needed to resipond to these questions. The LOI was submitted to the SSC Laboratory on Novernber 30, 1991.

Lane and a group of local theorists (Golden, Simmons, E. Carlson (Harvard) and L. Randall (MITT)) addressed the question of GEM's complementarity to SDC by studying two processes that require extended running at ultrahigh luminosity, $\mathcal{L}=10^{34} \mathrm{~cm}^{-2} \mathrm{~s}^{-1}$, and which benefit from GEN's very precise electromagnetic calorimetry and robust, highprecision muon system [35]. Using the PYTHIA / JETSET event generators, they studied the signals and backgrounds for two models each of $Z^{\prime 0} \rightarrow e^{+} e^{-}, \mu^{+} \mu^{-}$(for $M_{Z^{\prime}}=4 \mathrm{TeV}$ ) and of $\bar{q} q \rightarrow \mu^{+} \mu^{-}$assuming quarks and leptons are composite at the scale $\Lambda=25 \mathrm{TeV}$. They found that the heary $Z^{\infty}$ could be well-studied in one to two years, and quark/lepton subsiructure in just one year, by taking advantage of GEM's largely undiminished capabilities for high-pr physies at $\mathcal{C} \simeq 10^{34} \mathrm{~cm}^{-2} \mathrm{~s}^{-1}$. In particular, by measuring the $Z^{10}$ width in the $e^{+} e^{-}$mode and the final-state angular distribution in the $\mu^{+} \mu^{-}$mode, it 
is possible to distinguish different $Z^{\prime 0}$-models. They also showed that the muon angular distribution can be used to distinguish models for quark-lepton substructure which induce contact interactions with different chiral structures. Finally, with less statistical precision, the GEM detector could discover a $Z^{\prime 0}$ with mass in excess of $10 \mathrm{TeV}$ and quark/lepton substructure at the scale $\Lambda \simeq 40 \mathrm{TeV}$ in one year at $\mathcal{L}=10^{34} \mathrm{~cm}^{-2} \mathrm{~s}^{-1}$.

In January 1992, the PAC reviewed the GEM LOI and recommended that the collaboration proceed toward the preparation of a Technical Design Report. In its report, the PAC expressed concern about various aspects of the GEM design and of the physics simulations presented in the LOI. The areas of concern in the physics simulations were:

- Details of the search for an intermediate mass Higgs $\left(80 \mathrm{GeV}<M_{H}<180 \mathrm{GeV}\right)$ in $\gamma \gamma$ and $Z Z^{*} \rightarrow e^{+} e^{-} e^{+} e^{-}$modes.

- GEM's ability to discover the intermediate mass Higgs produced in association with $\bar{t} t, W^{ \pm}$or $Z^{0}$, and how this ability compared with that of the SDC detector.

- GEM's ability to tag heavy flavors, such as the top quark, by tagging so-called inclusive b-decay muons, buried in hadronic jets.

- The credibility of the missing- $E_{T}$ for a pair-produced gluino.

- Lepton identification and measurement at $\mathcal{L}=10^{34} \mathrm{~cm}^{-2} \mathrm{~s}^{-1}$. Although this was addressed in the LOI (see above), the $Z^{\prime 0}$ and substructure studies were repeated with greater depth.

Lane. Paige and K. MacFarlane (SSCL) organized a group of about 30 GEM collaborators (several new since the submission of the LOI) to respond to the PAC's concerns fand to begin preparation of the physics section of the TDR. This group met monthly at the SSC Laboratory to formulate plans and discuss progress.

The first major accomplishment of this effort was the development of what is called the FAST model of the GEM detector [41]. While it would be desirable to carry out full GEANT simulations of the detector for the study of physics processes, such simulations are far $t(x)$ slow to be practical. Indeed, the GEM design now changes significantly on a time scale short compared to that required to carry out such full detector simulations. Thus, the FAST simulation of GEM was developed for physics studies. FAST uses parameterizations of the responses of each of the major GEM subsystems to all particle stimuli of interest. These parameterizations are derived from full GEANT simulations of these subsysterns as 
described in the GEM Baseline 1 document [42]. As the GEM Baseline evolves, so will the FAST model. On most workstations being used for GEM physics studies, FAST analyzes generated events at the rate of about 1 per 3 seconds.

The FAST model of GEM was used to prepare the response of the GEM Physics Group to the PAC [41]. This was submitted to the PAC at its meeting in Woods Hole, July 10-15.

\section{B.2 Proposed Research}

Lane will be on sabbatical from academic duties during the coming year and he expects to devote at least $2 / 3$ time to organizing and carrying out physics simulations for the GEM TDR. In broad outline, the processes that the Physics Group hopes to investigate are the following:

1. Standard-model Higgs boson $\left(H^{0}\right)$ signals and backgrounds for the mass range $M_{H}=$ 80 to $800 \mathrm{GeV}$.

2. Heavy flavor physics, including discovery of: a heavy, $O(250 \mathrm{GeV})$, top quark in standard and nonstandard decay modes; leptoquarks in the decay modes $b \tau$ and $b \mu ; a$ technicta in the it decay mode.

3. Jet physics, including the detector-related issues involved in the verification of a signal for quark substructure as an excess of jets at high-p .

4. Lopton physics at ultrahigh luminosity, including studies of: $Z^{\prime 0}$ and $W^{\prime \pm}$ mass, width and couplings; quark-lepton substructure in both $\mu^{+} \mu^{-}$and $\mu^{ \pm} \nu$ final states; colorsinglet technirho production and decay to $W^{ \pm} Z^{0}$.

5. Mlissing- $E_{T}$ physics in the search for supersymmetry, and for a very heavy Higgs boson in the mode $\ell^{+} C^{-} \nu \bar{y}$.

This is an ambitious list of physics topies and carrying out all these simulations in time for the TDR will require a considerable effort on the part of the GEM Physics Group. 


\section{Cosmology and Astrophysics}

\section{C.1 Finite Temperature Quantum Field Theory and Phase Transitions}

in the Early Universe

$\mathrm{Pi}$ and Cohen have been studying various issues in finite temperature quantum field theory, which describes equilitrim and nonequilibrium a pects of phase transitions in the early universe - key ingredients of cosmology.

Pi, Eboli and Jackiw have developed a calculational technique for studying the timeevolueion of a quantum field theoretic system in an external environment changing with time [43]. Observing that the formulation of quantum field tiseory in terms of causal Green"s functions is not well-suited to describing a system's time evolution from a definite initial condition, they developed a formalism within the field-theoretic Schrödinger picture. Their technique is based on a variational principle for the density matrix. In the Gaussian approximation, one is provided with tractable variational equations that determine the time evolution of physical quantities. These equations are the analogs of time-dependent. Hartrer-Fock equations and reflect some of the nonperturbative nature of the full quantum thersy:

Pi. Eloli and Pis former student, Samiullah, used these calculational techniques to analyze the dynanies during phase transitions in the infationary universe [44]. They studied the inflation dynamics under various initial configurations of the inflation-driving scalur field. Among other things, their results establish the validity of the standard picture of a "slow rollover" phase transition in the "new" inflationary scenario, contrary to the asiscrtion made in Ref. [45] that the picture of "the ball rolling down the hill" cannot arise from an initial thermal equilibrium.

Cohen bas considered the question of finite temperature corrections to the effective potential. For the most part, these corrections have been computed and used at the one l(x)p level: however at high temperatures, perturbation theory becomes inadequate due to apparent infrared divergences. Cohen has used a renormalization group approach to this problem, which avoids these divergences and restores perturbation theory at high temperature $\{40\}$. This improved perturbation theory has many interesting features- it is an expansion in two couplings rather than one: the usual zero temperature coupling, as well as a ratio of the temperature to a mass scale renormalized at high temperature. Using 
simple loop expansion arguments, Cohen showed that an expansion in these parameters is under control everywhere except near a phase transition. At a first order transition, if the curvature is large compared to the coupling times the temperature, this renormaliaation technique leads to an accurate picture of the potential. These finite temperature renormalization group techniques can be applied to a wide variety of problems, including, e.g.. systems with a large chemical potential.

Studies of phase transitions in the early universe are based mainly on the early work of Dolan and Jackiw and Weinberg [47]. They used the one-loop effective potential to describe quantum field theoretic systems in equilibrium with an external thermal bath. However, cosmological scenarios often depend on the detailed non-equilibrium dynamics before, during, and after a cosmological phase transition, and on the nature of the phase transition, i.e., whether it is frst or second order. Therefore, to establish the validity of cosmological scenarios, the information provided by the one-loop equilibrium effective potential is not sufficient.

The question of the order of a phase transition may be answered by studying the finite temperature effective potential beyond lowest order in perturbation theory. What is newded is an approximation scheme in which a large class of ordinary perturbation-series diagrams contributing to the finite temperature effective potential can be summed in a consistont manner. In the relativistic field theory at zero temperature such a technique alrealy exists - the effective action and potential for composite operators. This can be used to analyze, by variational techniques, dynamical symmetry breaking and coherent solutions to fold theory. $\mathrm{Pi}$ and Eboli are investigating a possible analog of this effective potential for finite temperature quantum field theory.

Coluen's development of the finite $T$ renormalization group described above makes possible reliable statements about the weak phase transition in certain models. Cohen and A. Celson will investigate questions of the order of the transition and the reliability of these calculations using the finite- $T$ renormalization group. This formalism may be applied to determine the order of the phase transition in sfrecific models. M. Camperi is earying out a mumerical integration of the renormalization group equations in a model field theory as a test of this procedure.

Cohen's high- $T$ formalism may have applications in heavy ion physics, where it is possible that the scattering has high temperature features. For example, the naive corn- 
putation of deep inelastic scattering needs to be performed in a high density environment. The large density provides a scale which can be resummed using the renormalization group technique described above.

\section{C.2 Weak-Scale Baryoqeriesis}

The question of the origin of the baryon excess of the observable universe is one of the most profound we face. Particle physicists have come to understand some of the ingredients necessary for generating the baryon excess: fundamental baryon number nonconservation (either explicitly or spontaneously broken), CP violation, and out-of-thermal-equilibrium processes [48]. In the late 1970s it was shown how these three ingredients could arise in the context of Grand Unified Theories (GUTs) and lead to a baryon excess in the present epoch [49]. Indeed, the presence of baryon violation and the fact that it leads to a baryon excess today remains one of the most attractive features of GUTs. However, it is difficult to obtain a baryon excess of the size observed today without excessively complicating or fine-puning the GUT. In addition, the absence of proton decay at the rate predicted by the simplest GITs has cast some doubt on this explanation of the origin of the baryon excess.

An important complication to the question of baryon violation involves directly the guestion of wak interaction symmetry breaking. In the standard model, the weak interactionm themselves provide a source of baryon violation - the weak anomaly. Even though the classical throry conserves baryon number $(B)$, quantum corrections (similar to quantum tumbling through a barrier) cause $\triangle B \neq 0 \mid 50]$. Because of the small weak coupling and the fact that this violation is a quastum tunneling effect, the size of $B$ nonconservation is cxtremely small, and cannot play a significant role in the origin of the observed baryon number of the universe. However, it has been suggesced that at the high temperatures present in the early universe, classical thermal diffusion could cause $B$-violation without the suppression of quantum tunneling [51]. If this is true, it would completely change the development of the baryon excess during the evolution of the universe through weak scale temperatures. In fact, if the universe is near thermal equilibrium at this tixue, as is experted, then this source of B-violation would wipe out any baryon excess generated at earlier times. The argument is very simple: It the $B$-violating interactions are large cnough to be in thermal equilibrium, they will favor reactions which decrease $B$, since $B=0$ is the equilibrium value. Thus, the GUT scenario would no ionger produce a baryon excess! Scveral ideas have been proposed for avoiding this conclusion. One such 
idea is the production of $B-L$ excess rather than just a baryon excess [51]. Since the weak anomaly doe not violate $B-L$ symmetry, baryon number violation at the weat scale will no longer completely wash out excess baryon number. Thus, some baryon excess will remain, although somewhat smaller than the original $B \ldots L$ excess.

In their early studies of baryogenesis, Cohen and Kaplan attempted to move away from the GUT scenario for the generation of the baryon asymmetry. They developed a technique that, while still requiring fundamental baryon violation, manages to do away with the constraint of fundamental CP violation [52]. In addition, the requirement of departure from thermal equilibrium was avoided by having a scalar field far from its equilibrium value. In this model, baryon number is an approximate symmetry of the theory at high temperatures. As the universe cools, $B$-symmetry is broken spontaneously. This implies the existence of a pseudo-Goldstone boson, the "ilion", which couples to the baryon current. As this phase transition is passed through, the ilion field finds itself removed from its cquilibrium value, and begins to roll slowly towards its potential minimum. As it does, its intcraction with ordinary quarks and antiquarks lowers the energy of quarks relative to antiçuarks. This means that thermal processes will increase the number of baryons relative in antilaryous. As the universe continues to cool, B-violating processes eventually go out of equilibrium, and the baryon asymmetry is frozen in. As the universe cools still further, the ilion finds its true minimum, ceases to roll, and quark energy levels return to their normal ralue. The low energy world of this theory is just the standard model, and the interactions which have given rise to the asymmetry are unobservable. This technique of produrcing an effective chemical potential through the dynamics of scalar fields is called "spmentancous baryogenesis".

Cohen. Kaplan and Nelson have used spontaneous baryogenesis to construct models that can produce a baryon asymmetry at the weak phase transition. In Ref. [53] they developed a new method for generating a baryon asymmetry that, rather than avoiding fast weak interaction baryon violation at high temperature, makes direct use of it. The key problems for using weak baryon violation are to maintain departure from thermal equilibrium at the weak scale and to find a source of CP violation to drive baryon violation in the right direction. In one model, the departure from equilibrium was accomplished by having the weak phase trrnsition be weakly first order. The phase transition then occurs chrough bubble nucleation, with a non equilibriurn separation of phases. The $C P$ violation 
arises from a mass matrix for neutrinos. Neutrinos are massless outside the bubbles, but massive inside due to an electroweak symmetry violating vacuum expectation value. As neutrinos outside these bubbles interact with the wall, they are reflected. The reflected neutrinos are converted into baryons via anomalous weak inte actions. Net baryon number is developed if the reflection of neutrinos from the bubble wall is greater than the reflection of antineutrinos. It was shown that this could occur with the right magnitude to generate the observed baryon asymmetry if the tau neutrino mass is approximately $25 \mathrm{MeV}$. This value is in a range that can be sought at a tau-charm factory.

Cohen, Kaplan and Nelson applied this idea to a standard model with extra Higgs doublets [54]. Nothing in the model of Ref. [53] depended in principle on lepton number. If one can produce any left-handed fermion number excess, then anomalous weak interactions can convert this into a baryon asymmetry. Most left-handed fermion numbers in the standard model are not conserved; left-handed top quark number suffers the largest violation. Provided there is sufficient CP violation in the interaction between the top quark and the expanding bubble wall, a large left-handed-top asymmetry will be reflected back iuto the region where anomalous weak interactions are occurring, and will lead to a large baryon fasmmetry. This produces an asymmetry sufficiently large to explain the one observed today: This model has interesting experimental consequences aside from the baryon Hiymmetry. including the existence of extra scalars and new sources of $\mathrm{CP}$ violation.

Finally: Cohen and Manohar reconsidered previous estimates of the rate for anomalous Herak interactions at high temperature by studying massless QED [55]. This theory has an anomalous charge which fluctuates rapidly. They investigated the question of whether this fluctuation is related to a relaxation rate. Of course, due to a lack of topology in QED, there can be no relaxation. But the way this non-relaxation occurs is interesting. There is t conserved charge, although no corresponding conserved current. This is the axial charge of the fermions plus the linking number of the magnetic field. Thus, if two magnetic flux loop: unlink, they produce fermions with net axial charge.

Cohen will continue working on the generation of the cosmological baryon asyrnmetry. Although the paradigm and explicit models he presented with Kaplan and Nelson serm to have the right features, many of the computations used so far are quite crude. A better understanding of non-equilibrium effects involving the fermions is necessary to obtain a more quantitative estimate of the baryon asymmetry produced. Ideally, some sort of 
Boltzmann equation could describe the system even out of equilibrium. The rate equations used previously are only an impressionistic version of these more detailed equations. Work is in progress on this formulation of the problem, especially the question of Debye screening $[122]$.

Cnhen and Nelson are investigating baryogenesis in supersymmetric models. In order for the minimal model to produce the observed asymmetry, all of the parameters (such as the gaugino masses, higgsino masses, phases, etc.) must lie within very narrow ranges. This yields a rather definite prediction for electric dipole moments. In non-minimal models (for example the addition of a singlet scalar) the constraints are much less severe. Investigations by S. Myint indicate that a first order phase transition is quite natural in this case, and the baryon asymmetry is very easy to produce.

\section{C.9 Relic Particles}

If technicolor is responsible for electroweak symmetry breaking, there will be technibaryons analogous to the proton in QCD. These particles will carry technibaryon number and could be stable on the timescale of the age of the universe. Chivukula and $T$. Walker anklyzed the cosmological consequences of the existence of stable technibaryons [56]. They showed that the number of technibaryons produced during the Big Bang is large, at least as great as one technibaryon per $10^{7}$ baryons. They also showed that, in many technicolor moleis, the lightest technibaryon is stable, electrically charged, and would be present on the earth in numbers ruled out by searches for superheavy isotopes. They concluded that any technicolor theory in which the lightest technibaryon is stable and electrically charged (except for charges $-1,+2$, and +7 ) is excluded.

If the "dark matter" of the universe consists of a diffuse collection of elementary particles, it is usually assumed that they must be weakly interacting. This orthodoxy was challenged by De Rujula, Glashow and Sarid [57], who suggested that dark matter consists of extremrly massive charged particles, called "champs". Since conventional searches for dark matter particles rely on their interacting weakly with ordinary matter, these searches do not apply to champs. Chivukula, Cohen, S. Dimopoulos and Walker analyzed the constraints on strongly interacting dark matter arising from the observed properties of interstellar clouds [58]. They showed that, if champs formed the galactic halo, they would heat interstellar clouds to temperatures higher than observed. Therefore, champs are excluded as the prime constituent of the galactic halo. 
In the standard relic-abundance calculation of the production of stable particles in the early universe, it is assumed that the net number of baryons, leptons, and any other particle with a classically conserved quantum number remains unchanged. As we have discussed, baryon and lepton number are not separately conserved because of the weak anomaly and, at temperatures abs: $100 \mathrm{GeV}$, there may be processes in equilibrium which change $B$ and $L$. Chivukula, S. Barr and E. Farhi considered the effects of anomalous weak processes during the early universe in extensions of the standard model [59]. They showed that, if there is a new particle type whose number is conserved up to the weak anomaly, the number of new particles produced during the Big Bang will be proportional to the number of baryons present in the universe today. The constant of proportionality depends on the relationship between the temperature $T$ at which the anomalous processes fall out of equilibrium and the mass $M$ of the particle in question. If $T \geq M$, the constant of proportionality is of order one; if $T<M$ the constant is of order $\exp (-M / T)$. They applied these results to the production of technibaryons and showed that it is conceivable that, if they are electrically neutral, they form the dark matter of the universe.

Chivukula and Selipsky are collaborating on an extension of previous work [56] on the behavior of very massive charged particles in the early universe. This has implications for new degrees of freedom (such as technicolor) to be sought at the SSC. After the nucleosyn" thesis era, negatively charged, color-neutral particles would electrostatically bind protons and alpha particles, and appear today as TeV-mass neutral or positively charged nuclei. Obtaxining cross sections for the bound states' rate equations requires the Schrödinger equation to be solved numerically, since the relatively large nuclear charge radius means that the states are not hydrogenic. Then one can follow concentrations of the species of interest through freeze-out to the present time, and determine the prospects for terrestrial observation of relic technibaryons.

\section{C.t Astrophysical Objects}

Cohen and Kaplan have constructed an exact solution for the gravitational metric around a global cosmic string [60]. The metric for a gauged string is simply a flat metric and an angle deficit. For a global string it is quite different, since the energy density is not confined to the core of the string, but extends out to arbitrarily large distances. The 
resulting metric has a rich spectrum of particle orbits and, most interestingly, a singularity far from the string itself. This may be counterexample to the cosmic censorship hypothesis.

Selipsky's Ph.D. research before arriving at Boston University involved the invention of Q-stars, macroscopic bags of particles which are sources for a field which self-consistently binds them. Fermion Q.stars were proposed by Selipsky, Bahcall and Lynn as candidates for neutron stars [61]. The key features are their being held together by nuclear rather than gravitational forces, and their very large maximum stable mass resulting from a new equation of state below nuclear density. Thus, very small bound objects are possible (and might be candidates for dark matter), as well as neutron stars exceeding the 3.5 solar-mass limit for collapse to black holes. Depending on the nuclear theory parameters, very dense and very rapidly rotating stars could exist, instead of very massive ones [62]. 


\section{New Field-Theoretic Models}

Theoretical physics has enjoyed renewed vitality in recent years, due largely to the challenges presented in understanding string theory as a mode! of fundamental particle physics. There have been both new developments and new insights into old problems. Researchers at Boston "Yniversity have been active in several areas of mathematical physics. For discussion purposes, these are grouped below into the categories of Chern-Simons theory, higher-dimensional field theory, string theory and conformal field theory, although there are unifying themes in all of these approaches.

\section{D.1 Chern-Simons Gauge Theories in $2+1$ Dimensions}

Several of the theorists at Boston University have been working on Chern-Simons gauge theories in three dimeusional space-time. These theories possess pedagogical interest, teaching useful lessons that may be relevant to the physical four-dimensional world. They are also mathernatically fascinating, giving rise to effects and structures that invite further study: Moreover, they can have a physical role in descriptions of actual laboratory processes that are confined to a spatial plane. Tis quantum Hall effect and high- $T_{c}$ superconductivity - two planar phenomena - are analyzed in terms of Chern-Simons dynamics [63].

Quantum mechanics of N-charged particles interacting through $U(1)$ Chern-Simons gauge fields has not been solved except for the two-body problem. As an approach to the general $\mathrm{N}^{\prime}$ body problem, $\mathrm{Pi}$ and Jackiw constructed a nonrelativistic field theory model which is a second quantized description of nonrelativistic $\mathrm{N}$-body quantum mechanics. On the classical level, a gauged nonlinear Schrödinger equation emerges in this model. They found explicit, two-dimensional static, self-dual solutions that satisfy the Liouvill aquation [04]. The results of $\mathrm{Pi}$ and Jackiw show that, contrary to assertions in the literature that "the effect of the Chern-Simons terms is to transmute the statistics of the particles and to do nothing else" [63], their explicit soliton solutions vividly demonstrate that the ChernSimons term supports nonperturbative excitations whose role in quant um field theory still needs to be further explored.

$\mathrm{Pi}$ and Jackiw have studied other properties of their model [05]: its obvious and hidden symmetries, its relation to a relativistic field theory $(66,67)$ and its supersymmetric formulation. 
A complete analysis of the full, time-dependent, gauged nonlinear equation on the plane has not been achieved thus far, and it is not known whether the time-dependent system is as completely integrable in $(2+1)$-dimensional space-time as is the static system in 2-dimensional space. However, some time-dependent solutions have been found. All of them are related to the static, self-dual solutions by space-time transformations. A Galilean boost and a conformal boost, both symmetry transformations of their model, introduce time-dependence in the static soliton profile while preserving the validity of the equation of motion. More interesting time-dependent solutions are obtained when these spacetime transformations are generalized. One can construct space-time transformations which are not symmetry transformations, but map their system to other interesting dynamical models, such as external harmonic and constant magnetic field problems [68-70]. Therefore, when these transformations are applied to the static or boosted self-dual solutions, the resulting time-dependent solutions describe the motion of the self-dual solitons in the presence of external magnetic field or harmonic force. These solutions are periodic in timc; $\mathrm{Pi}$ and Jackiw were able to quantize them by the Bohr-Sommerfeld procedure and obtained semi-classical energy quantization.

$\mathrm{Pi}_{\mathrm{i}}$ and her collaborators have further generalized the abelian theory to nonabelian theorim [i]]. They have shown that the self-dual nonrelativistic, nonabelian systems lead to the Toda hierarchy of integrable equations and thus the nonabelian Chern-Simons theories provide a unified framework for these equations.

The work of Rohm's student, M. Camperi, on Chern-Simons gauge theories has focused on the connections between topological field theories in three dimensions and conformal ficld theories in two dimensions. His publication [72] deals with an exact expression for the partition function of the three-dimensional nonabelian Chern-Simons gauge theory. On the one hand this result confirms the results of low-orders in perturbation theory. It Also allow's the construction of thm large-N limit of the theory, which exhibits an unexpected duality between weak- and strong coupling.

Work is continuing on various aspects of the Chern-Simons theory and its applications to anyons. Pi and her student, G. Amelino-Camelia, are working on the non-relativistic model, investigating perturbative asperis of the quantum theory of nonrelativistic bosons with statistical interactions. 
$\mathrm{Pi}$ and collaborators are investigating the complete integrability of the Abelian ChernSimons model. There are hints that the model is integrable since, in a particular ChernSimons theory, some exact soliton solutions were found [64]. An integrable theory with a Chern-Simons term would be of great use in understanding the often-subtle properties of anyons.

One straightforward way of determining whether or not a model is integrable is to simulate the system numerically. The most obvious signature of this is completely elastic scattering. Graduate student $R$. Strilka is simulating numerically scattering processes of Chern-Simons solitons to look for integrability. If the model is integrable, then the numerical results may provide a guide to finding exact analytic properties of the model. For example, the extra conserved charges underlying the integrability should be discoverable numcrically. Success would be especially remarkable in view of the dearth of integrable 2+1-dimensional models.

\section{D.2 Higher-dimensional Field Theories}

Rohm has applied higher-dimensional topology to string theory by exploring the interrelationship of global gravitational anomalies, exotic differential structures, and gravitational topological defects. These all involve the study of the subtle effects of changing differential structures on the space-time manifolds of higher-dimensional quantum field theories. Such structures occur in the consideration of global anomalies in higher-dimensional gravity throries [73], and may give rise to localized topological defects [73, 74]. Although there is no convincing relation of these objects to four-dimensional physics, analysis of these objerts is actually rather more straightforward than the corresponding four-dimensional objects (duc to some famous and remarkable features of four-dimensional topoiogy). Contrary to the usual situation, one can view this analysis of differential-structure 'defects' in higher dimensions as a warmup to the four-dimensional problem.

Rohm has also applied some of these techniques to a collaboration with B. Grinstein and J. Preskill on the general problem of defining fermion fields on nonorientable manifolds. (In analogy to the or:entable case, in which the different possibilities of extending the $S O(n)$ symmetry to fermions are referred to as Spin structures, in the nonorientable $O(n)$ case these are referred to as $\mathrm{Pin}$ structures). This question was of interest in the two-dimensional casc $[75]$ for the treatment of the open-string theory where the string Feynman diagrams 
include nonorientable surfaces; the extension of this work to higher dimensions [76] is interesting mainly because of the parallels with the properties of fermions on orientable manifolds.

\section{D.9 String Theory}

The discovery of the superstring anomaly cancellation by M. Green and J. Schwarz [77] in 1984 triggered a tremendous amount of interest in string theory by the particle physics community. They showed that the open-superstring theory with $S O(32)$ gauge group was the only version of the theories known which contained Yang-Mills gauge bosons and was (apparently) free of anomalies. There was a loophole, since the low-energy anomaly cancellation conditions urere also satisfied by supergravity theories based on the gauge group $E_{8} \times E_{8}$. This led to the discovery of the heterotic string [78] by a collaboration which included D. Gross, J. Harvey, E. Martinec and Rohm. This theory had several novel features with respect to previously-known string theories; in particular, the assignment of different quantum numbers to left-moving and right-moving fields on the string made it possible to realize $N=1$ supersymmetry and Yang-Mills gauge symmetry simultaneously for a closed-string theory. These novel features have figured prominently in much of the sulsisquent development of string theory.

The two most important problems in relating ten-dimensional string theories to low. encrgy physics are the nature of the compactification from ten to four dimensions and the merhanism responsible for supersymmetry breaking. Rohm has had several projects which were attempts to understand the relationship between compactification and supersymmetry breaking. The first attempt [79] was a mechanism for supersymmetry breaking in compactifications of the original Green and Schwarz superstring theories on toroidal manifolds through ewisted boundary conditions. (To use anachronistic terminology, this is a special type of orbifold [80], in which we divide the torus by a symmetry group which arts freely but does not preserve supersymmetry.) Rohm next studied this problem in an investigation with M. Dine, N. Seiberg and E. Witten of a possible mechanism for dynamical supersymmetry breaking in Calabi- lau compactifications of the $E_{8} \times E_{8}$ beterotic string [82]. With plausible assumptions about the low-energy dynamics of string theory it was demonstrated that the second $E_{8}$ may becorne strongly-interacting, resulting in the formation of a gluino condensate which breaks supersymmetry. 
As an outgrowth of this problem, Rohm collaborated with Witten [83] on the need for and consequences of quantization of antisymmetric-tensor-field expectation values in string backgrounds. This paper exhibited a novel mechanism for tunneling between the quantized values of the antisymmetric tensor field, as well as the existence of some new soliton-like solutions of the compactified theory. The work on gluino condensation also led Dine and Seiberg [84] to discover a serious problem with string phenomenology, namely that attempts to stabilize the string vacuum against expansion of the internal manifold generically imply coupling constant values near unity, due to the identification of coupling constants with background fields in string theory.

Rohm has recently concentrated on the use of conformal field theory techniques in the study of compactified string theories. Such techniques are crucial in understanding the physics of conpactifications of small radius (comparable to the string scalc) where the sigma-model coupling becomes strong. Initial studies of string theories compactified on Culabi-Yau manifolds indicated that models with moderate or large radius of the internal dimensions could no: be phenomenologically successful. Although some properties could be scliably computed in the $1 / R$ perturbation theory, there were also indications of significant differences between small-radius solutions and the predictions of perturbation theory. It is then fortuitous that there are exactly-soluble conformal field theories [85] which correspond to compactification on certain Calabi-Yau manifolds of a fixed small radius. Recently stu ly' of perturbations around these solutions [80] demonstrated the existence of a small-radius boundary to the moduli space of Calabi-Yau compactifications. Perturbations away from this boundary are distinguished by the axion mode: if one approaches this point from a large-radius compactification and passes through, instead of gaing to smaller radius one approaches a large radius compactification with a differene vacuum for the axion field.

In contrast to the questions of compactification and supersymmetry breaking in string theory, which attempt to recover farniliar physics results from a more fundamental theory, there are also some difficult and unsolved problems pertaining to the basic structure of the throry. In particulas, the standard formulation of string theory allows calculation of only on-shell scattering amplitudes; more satisfactory formulation would define off-shell quartities and fully explore the symmetry structure of the quantum theory.

One approach to defining and computing off-shell amplitudes in string theory was developed by Cohen, G. Moore, P. Nelson and J. Polchinski [87]. The usual formalism 
involving insertions of vertex operators at points on the world sheet only allows for the computation of on-shell S-matrix elements. By using the functional integral on world sheets with boundaries, they were able to compute amplitudes off the mass shell. This technique implements Feynman's original sum over histories prescription; the functional integral is then a sum over all possible "string paths" that can connect these boundaries. The simplest application of this technique involves the computation of the two-point function, the string propagator. This prescription can be used in one dimension to obtain the usual propagator for free particles. The extension to string theory leads to a propagator with the usual features: as a function of $k^{2}$ it has poles corresponding to the physical string states. Since string theory contains gravity, the generalization to higher n-point functions requires constructing generally covariant amplitudes. We showed that amplitudes satisfying these conditions exist, and reduce on shell to the well known amplitudes obtained by the vertex operator prescription.

String ficld theory also attempts to give a well-defined meaning to off-shell calculations in string theory. One problem with known formulations of string field theory is the nontrivial dependence on background geometry; although the interaction term of the action for open-string ficld thenry can be made background-independent through field redefinitions, the hinctic term involves the background metric in an essential way. Some progress was made by G. Horowitz. J. Lykken, A. Strominger and Rohm [88], which demonstrated that in open string theory one can obtain the standard action from one consisting of only the intrraction term, by a suitable shift of the string field. This work had a number of interesting consequences (considered by Horowitz and Strominger, among others), including asmariativity anomalies in string products and their resolution, the definition of closedstring cubic actions through open-string fields, and novel (indeed, bizarre) backgrounds for string therry [89-91].

Rohm is continuing investigations of compactified string theories. The first scheme for obtaining four-dimensional physics from the ten-dimensional heterotic string was compactificreion of six of the dimensions on a compact. Ricci-fat complex manifold, a so-called Calabi-lian manifold. Although many other compactification schemes have since been discuss:d. these still possess many attractive features; recently a construction was shown to yicid thousands of distinct compactifications as varieties in weighted-projective spaces ( $\left.H C P^{M}\right)$. Rohm, and his student, V. Pitsis, began an attempt to find phenomenologically 
sppropriate models: that is, a net number of three light fermion families, together with a non-simply-conneclied manifold suitable for Wilson-line symmetry breaking. In searching for ways to deal with the technical difficulty of resolving the orbifold singularities in order to ensure that the discrete symmetry acts freely, we began investigating wheiher there is a geometrical way of incorporating the blowing-up moduli, generalizing a few known examples where a $W C P^{n}$ compactification can be related to arinther compactification in which the orbifold singularities are absent.

\section{D.\& Two-Dimensional Ficld Theory}

Since the mid.1980s, there has been enormous progress in understanding two-dimensional field theory: Much of this suress rose out of the study of conformal field theory, which is a major part of string theory. These field theories describe a system at its critical point, where the scale invariance is part of a larger symmetry, the conformal symmetry. These developments have many applications outside string theory, and in models where one breaks the conformal symmetry by adding a mass term. All of these methods are wluable for the general study of field theory, because one does not generally use the standard perturiation theory of Lagrangian field theory. In fact, in most cases, one does not men know the Lagrangian of the field theory, but finds nonperturbative results. Since there are gat many problems in particle physics which at the moment are unsolvable by the old mothods, it seems an opportune time to hunt for new methods. As we will detail below, these new techniques provide not only fresh perspectives but also relate to real condensed-matter systems.

Much has been learned about field theory by studying statistical-mechanical lattice models such as the Ising model. since in the continuum limit lattice models are often described by feld theories. One important property of the Ising model is its Kramers-Wannier duality, which relates a low-temperature model to a high-ternperature one. Generalizing the "orbifold" procedure in conformal field theory for modding out a theory by a symmetry to obtain a new one, P. Fendley and P. Ginsparg showed that such duality is in fact a much more general property of lattice models and their corresponding field theories. In Refs. [02, 93]. procedure for obtaining a new lattice model from a given one was explained, and it was shown, for example, how the partition functions of the two were related. For instance, the A-series and D-series of minimal models in conformal field theory (and its massive perturbations) are related in this manner. It is especially interesting because this 
procedure preserves the integrability of a model. However, this procedure is good for nonintegrable models and also those in three and four dimensions, whiere it applies to models with discrete gauge symmetries [94]. These models give a way of providing quantum hair for a black hole. Future :mak involves fully understanding this structure.

Another area where the advances in understanding conformal field theory have had more general applications is the study of integrable models. One reason that so much can be learned about coniormal field theories is that the conformal symmetry is infinitedimensional, making the model integrable. For particular perturbations these models remain integrable. One striking property of such massive models is that the scattering is completely elastic (particles essentally go through, allowing only phase shifts and the exchange of qu.ntum numbers), and the $n$-body $S$-matrix factorizes into products of twobody ones. The enormous number of constraints this requires means that the exact $S$ matrix can often be conjectured.

In collaborations with S. Mathur, C. Vafa, N. Warner, W. Lerche, and K. Intriligator, Fendley studied a tariety of properties of integrable models with $N=2$ supersymmetry. Below wa will discuss applications of these models to condensed-natter physics; here we stress the formal aspects. For the most relevant perturbation of the $N=2$ superconformal minimal models, the soliton spectrum, the exact S-matrix and the conserved currents Lave bern found $[05,96]$. The nonrenormalization theorems of $N=2$ theories enable the determination of the exact masses and the bound states of the solitons; in most integrable moxlels they are conjectured when requirixg the consistency of the exact S-matrix. The $P=2$ models have many similarities to the the $N=0$ models, so this study gives valuable intuition into the non-supersymmetric caje.

From the exact S-matrices calculated above, one can find nonperturbative thermodynamic information, since all of the properties of a dilute gas are derivable from the S-matrix. There is a very elegant technique for deriving such pr zperties, often known as the thermodynamic Bethe ansatz [99]. These analyses enable the determination of the Casimir encrgy (lowest eigenvalue of the Harniltonian) as a function of the mass and the volume of the systern. This provides a very useful check on the conjectured S-matrix, because in the zero-mass limit, the Casimir energy is related to the central charge of the corresponding conformal field theory. It also provides a sort of 'c-theorem', providing a 
way to quantify the senormalization-group flows between different conformal field theories. This calculation has been done for the $N=2$ scattering theories discussed above [96].

A striking result of these studies of $N=2$ theories has been the discovery by Cecotti, Fendley, Intriligator and Vafa of a new supersymmetric index, $\operatorname{Tr} F(-1)^{F} \exp -\beta H$, where $F$ is the fermion number [120]. It is an index in the sense that it depends on only a finite number of parameters of the theory. This index is related to the geometry of the vacua (Berry's curvature) and satisfies an exact differential equation as a function of $\beta$. As with Witten's index $\operatorname{Tr}(-1)^{F} \exp -\beta H$, the calcr'ation gives non-perturbative information about the theory and its spectrum. For integrable theories the results of Fendley and Intriligator mentioned above can be usea to compute the index thermodynamically. The equiralence of these two results implies a previously unknown and highly non-trivial equivalence of a set of coupled integral equations with these differential equations, among them Painieve III and the affine Toda equations.

Fendley and Saleur have applied these results to the physical system of self-avoiding polymers on a cylinder of circumference $\beta$ [121]. The index corresponds to the partition function of a single polymer wrapped around the cylinder, which enables the calculation of the exact scaling function for the number of such configurations in the continuum limit. In addition. Fendley and Saleur also performed the analogous calculation for a single polymer not neressarily wrapped around the cylinder, and derived the ground-state energy conjectured by Zamolodchikov for the perturbed minimal models.

A surprising result found by Fendley and Saleur was the behavior of the low-temperature poinger phase, where the polymers fill space. With numerical study of the corresponding lattice model, it was found that this phase has a Casimir energy which rises and falls as the mass of the theory is changed. This was surprising because there were numcrous conjectures that all such functions must monotonically decrease with increasing , mass: i.e.. it was thought that non-unitary theories obey a $c$-theorem as do unitary ones. This result indicates that there is a qualitatively new structure in this phase, which is now being studied asalytically.

Fendley has shown how excited-state energies can also be derived from the thermodyanamics in any theory with a discrete symmetry [09]. Basically, one changes the boundary conditions to project out the ground state. In lattice-rrodel terms, these are exact calculations of the interfacial tensions. This provides as understanding of operator flow between 
conformal field theories, because these excited states are created by operators acting on the ground state.

Many integrable models can be described by a suitably-truncated Toda field theory. Toda theories are generalizations of Liouville and sine-Gordon theories, and have many interesting mathematical properties which have made them widely-studied. $N=2$ models are related to Toda theories, revealing a hidden supersymmetry of these theories [97]. This supersymmetry is a local [98] and hence useful example of the general quantum. group symmetry of the Toda theories. This correspondence enables the determination of off-critical conserved currents for a number of $N=2$ minimal-model perturbations as well as for more general coset models.

Fendley is also considering the application of information derived from the exact solutions to problems in condensed matter physics. While two-dimensional $N=2$ supersymmetric theories are mainly of formal interest in particle physics, they can be experimentally relcrant in condensed-matter physics. One interesting aspect of the $N=2$ moriels discussed above is that the solitons have fractional fermion number $[96 \mid$. This is possible because thar Dirac muation in the presence of a soliton has a zero-mode solution invariant under charge-conjugation. The phenomena of fractional fermion number in $1+1$ dimensions occurs in physical polymer systems where one extra bond (fermirn) can be distorted into $n$ whlitons asud. thus, each soliton carries fermion number $1 / n$. Therefore, this analysis may be saluablo for studying these experimentally-realizable systems. One cannot measure the S-matrix or the Casimir energy directly, but many other things about these $N=2$ systems may be observable - for example, the (exactly-known) critical exponents. Fendley and K. Intriligator (Harvard) are investigating the physical applications of these models.

The self-avoiding polymer is another physical system with $N=2$ supersymmetry (in any dimension). Recently. Fendley and Vafa observed that, in two dimensions, this is precisely the "twisted" $N=2$ supersymmetry which appears in topological field theories. This connertion could be very fruitful. because many properties of polymers may be essentially topological. This connection has enabled $H$. Saleur to relate the minimal series of $N=2$ models to the multicritical polymer hierarchy. One very interesting direction is to understand how the many known properties of the $N=2$ and the topological systems theories manifest themselves in the polyrner systems. A related investigation is to understand 
the behavior of the low-temperature polymer phase analytically; Fendley and Saleur have shown numerically that this phase has a qualitatively new structure.

\section{E. Nonperturbative Investigations of Quantum Field Theories}

A major development in particle theory research has taken place during the last decade through the introduction of computer simulation techniques [100-102]. Numerical investigations, normilly carried out in the context of the lattice regularization of quantum gauge theories [103], have made it possible to calculate several important observables, which, because of their non-perturbative nature, could not be determined following traditional analytical techniques. Within the Boston University particle theory group, faculty members C. Rebbi and R. Brower, research associates E. Myers, S. Huang, J. Potvin and E. Vicari, together with several students and visitors, have actively pursued this line of investigations.

Major topics of research during the last few years have been the inclusion of fermionic degrees of freedom in lattice calculations of the hadronic spectrum, the quantization of a therry on a null-plane lattice, the calculation of the surface tension in finite temperature QCD. multigrid methods for quark propagators and other acceleration techniques, the evolution and interaction of cosmic strings and superconducting vortices and matrix models for 2-d gravity and effective string theories. Research on the first two topics above has been at liast temporarily concluded, and since the major results have already been outlined in a recent proposal to DOE, we will not review it here. The other subjects represent areas of investigation where, at various stages, research is still going on. These will be concisely summarized bolow.

Numerical investigations have provided evidence that at high temperature, Quantum Chromodynamics (QCD) undergoes a transition from a hadronic phase of confined quarks to a Debye screened quark-gluon plasma phase. In the quenched approximation, where one neglects the creation and annihilation of virtual quark-antiquark pairs, the transition is weakly first order. Therefore, at the transition point the two different phases can coexist and the surface tension, i.e., the excess free energy due to the presence of an interface, becomes an important physical quantity. It determines the nucleation rate of 1 adrons as the quark-gluon plasma cools down past the transition and its value las implications for cosmology and high energy nucleus-nucleus collisions. In collaboration with J. Potvin, 
Rebbi has introduced a novel method for calculating the surface tension in a first order transition [104]. This has been applied to quenched QCD [105] as well as to models of interest in condensed matter physics [106]. In a collaboration involving also R. Brower, S. Huang and J. Ross, the method has been improved to include operators that correspond to a finite density of quark sources (the so-called Polyakov loops). This has permitted the calculation of the surface tension on a finer lattice, where previous calculations by the method of Potvin and Rebbi and by other independent techniques had failed as well as the verification of the "perfect wetting" properties of the transition [107-108]. The value of the surface tension that emerged from the calculation is approximately $6 \mathrm{MeV} / \mathrm{fm}^{2}$, a very small value on the typical scale of hadronic phenomena, which corroborates the weakness of the transition.

The numerical calculation of quark propagators is one of the most important computational steps in all QCD simulations. It is a fundamental ingredient for the determination of the spectrum of mesons and baryons and it is at the basis of all current methods for simulating full QCD (as opposed to QCD in the quenched approximation), where it also constitutes the most time consuming part of the simulation. Thus, the development of efficient methods for the calculation of quark propagators is of paramount importance for this entire ficld of research. This need is made even more pressing by the fact that, as one tries to improve on the calculation by moving to finer lattice spacings and larger lattices, critical slowing down makes the calculation of quark propagators even more time consuming. Working in collaboration with E. Vicari and R. Edwards, Brower and Rebbi have applied multigrid techniques to the calculation of quark propagators with the goal of overcoming critical slowing down. The research has progressed by incorporating increasing levels of difficulty (2-d to 4-d, Abelian to non-Abelian, bosonic propagators to fermionic ones) into the multigrid method and focusing on the physical reasons for critical slowing down. Substantial improvement of convergence has been achieved [109-112], but obstacles to the total elimination of critical slowing down persist and research on this topic is still in progress. In a different but similarly motivated line of investigation, Brower and collaborators have applied cluster algorithms to the acceleration of the simulation of bosonic systems. Successful results have been obtained for Higgs models and for a class of Abelian gauge theories (113-114). 
Cosmic strings and superconducting vortices are very interesting topological structures which share a common mathematical background provided by the Ginzburg-Landau or Abelien-Higgs model. While the properties of static vortices have been reasonably well under stood for many years, meaningful investigations of their dynamical properties, which to a great extent must rely on very large scale computing, have only recently become possible. At the same time, new questions on the role that cosmic strings may have played in the evolution of the early universe have made such investigations particularly pressing. Rebbi and collaborators have developed a code for the simulation of the classical motion and interaction of vortices, which takes advantage of the formalism of lattice gauge theories to preserve gauge invariance even in the discretized equations [115]. This code has been applied to several investigations of the interaction among vortices in two and three dimensions [116-117]. In particular, it has been shown that cosmic strings "intercommute," i.e., trade ends in a collision, and that aligned vortices undergo $90^{\circ}$ scattering in a central collision under a wide range of collision parameters.

Brower and collaborators studied unitary matrix models nearly a decade ago to understand features of the large $\mathrm{N}$ limit for a simplified model of QCD [118]. Recently Hermitian matrix models have been exploited as a way to sum random surfaces for string theories with contral charge $c \leq 1$, and many new techniques have been found as a consequence of the explosion of recent work starting with the discovery of the double scaling limit. In recent research conducted in collaboration with N. Deo, S. Jain and C.-I. Tan, Brower has studied the multitude of phases encountered in the double well single matrix model [119]. They have been able to augment analytical results by a simulation of the matrix model on the Connection Machine, formulated in terms of an effective action for the recursion cocfficients in the orthogonal polynomial formalism. By simultaneously exploring many different parameter values (on the order of $10^{3}$ ) a rapid numerical survey can be made and . many conjectured properties verified or rejected.

In the future, Rebbi, Brower and collaborators plan to continue some of the investigations outlined above and to pursue additional projects for some of which they have preliminary results. For example, several recent studies of surface tension have indicated that it would be valuable to have detailed information about its dependence on the curvature of the nucleating droplet. The study of such curvature dependence is proceeding in both quenched QCD (by Rebbi, Huang and Potvin), and in the Ginzburg-Landau model 
(by Brower, Klein, Potvin and Tamayo). In addition, recent work on hot nuclear matter suggests a weak first order transition. Thus the models currently used in the study of strong first order phase transitions need to be modified in a manner suitable to the description of weak transition kinetics. To this end, in the collaboration with Klein, Potvin and Tamayo, numerical simulations on the nucleation of domains in both the classical and the critical region of the Ginzburg-Landau model are being performed. In particular, these investigations seek to discover the correct prefactor which acts to modify the nucleation rate given by the standard exponential barrier penetration. Finally, for nucleation in QCD we also plan to investigate the important issue of going beyond the quenched approximation.

There is additional work on multigrid methods and matrix models still in progress, as well as ongoing research on cosmic strings. $R$. Strilka, a graduate student who has worked together with Rebbi on the simulation of cosmic strings and superconducting vortices, has recently extended the simulations to Chern-Simons theories. These theories have received a lot of attention as models for particle interactions with interesting topological properties and have also been studied, within the Boston University particle theory group, by $\mathrm{Pi}$, Fendley and collaborators.

Additional topics targeted for future investigations include the calculation of the paramepers for chiral lagrangians from first principle lattice calculations and the interfacing of per urbative QCD with non-perturbative lattice techniques. Results on these problems will have immediate and important phenomenological applications. Preliminary research on the derivation of chiral lagrangians and other effective lagrangians has already begun. In the fall of this year Yue Shen will join the Boston University particle theory group as research associate. He has been interested in the application of non-perturbative techniques to the study of the Higgs phenomenon and to physics in the $\mathrm{TeV}$ range, an area where he has already published valuable research results. It is expected that these topics will form the subject of future investigations and, indeed, that these will involve several members of the particle theory group in a collaborative research effort.

In related activities, Rebbi and Brower have played a major role in establishing and guiding the Boston University Center for Computational Science, which, with its Connection Machine and other facilities, provides support to a wide range of computationally based research, including a large part of the investigations outlined in this section. Rebbi is the Center's director and Brower is a member of its scientific council. 
Rebbi was a cofounder of the Topical Group, now Division of Computational Physics of the APS, for which he served as vice-chairman (1988-90) and chairman (1990-91) and was the organizer of the first general meeting, held at Boston University in 1989.

Brower was the principal organizer of a workshop on Multigrid Methods held at Boston University in April 1990.

Brower and Rebbi are members of the QCD Teraflop collaboration, for which Brower serves on the steering committee.

\section{F. Other Research Results}

Important research results have been obtained by our visitors during their stays at Boston University. Since they interacted with the researchers in this proposal and, to a limited extent, received support from the DOE grant, some of their research accomplishments are listed here.

T. De Grand and his student, M. Hecht, used the Connection Machine to analyze the amihilation patlern of topological defects in twu-dimensional classical $O(2)$ field theory thr amihilation of a vortex and an antivortex in spin waves. The problem is interesting both in the feld of cosmic strings and in condensed matter physics (for films of liquid crystals). They made a movie of vortex collisions, which provided useful insights into the properties of the system.

C. Korthals-Altes investigated methods to reduce finite size effects in pure gauge theory by sarying the type of boundary conditions, the coexistence of phases in the 3-state Potts model ("perfect wetting"), weak currents in staggered fermion QCD in connection with thrir anomalous Ward identities, and a Monte Carlo measurement of a possible Meissner effect in gauge theories.

R. Petronzio, working in collaboration with E. Vicari, investigated the definition of improved operators for the numerical simulation of lattice gauge theories. The idea is based on a microcanonical average of statistically independent link variables, which can be performed by an exact integration over part of those group elements that leave invariant the force acting on a given link. These ideas have produced a novel "heat bath" algorithm, the "over heat bath", where the compromise between a standard heat bath and a microcanonical update is realized dynamically. 
J. Potvin, in addition to the investigations carried out in collaboration with Rebbi and Brower, used the histograin method of Farrenberg and Swendsen to calculate the energydensity, the pressure and the speed of sound in the quark-gluon plasma, and also studied a novel method for a direct calculation of the scattering matrix from the Hamiltonian by lattice and Monte Carlo techniques.

J. Shigemitsu, while at Boston University, continued with her long-standing program of investigations on Higgs-Yukawa-chiral theories obtaining results on the possibility of decoupling unwanted fermion doublers in a variety of chiral models. She also pursued research on the light cone formulation: of a quantum field theory.

\section{REFERENCES}

1. S. Weinberg, Phys. Rev. D19, 1277 (1979); L. Susskind, Phys. Rev. D20, 2619 (1979).

2. E. Eichten and K. Lane, Phys. Lett. 90B, 125 (1980); S. Dimopoulos and L. Susskind, Nucl. Phys. B155 (1979) 237.

3. B. Holdo:n, Phys. Rev. D24, 1441 (1981); Phys. Lett. B150, 301 (1985); T. Appelquist. D. Karabali and L. C. R. Wijewardhana, Phys. Rev. Le't. 57, 957 (1986); T. Aprolquist and L. C. R. Wijewardhana, Phys. Rev. D36, 508 (198i); K. Yamawaki, M. Bando and K. Matumoto, Phys. Rev. Lett. 56, 1335 (1986); T. Akiba and T. Yanagida, Phys. Lett. B169, 432 (1986).

4. A. G. Cohen and H. Georgi, Nucl. Phys. B314, 7 (1989).

j. T. Appelquist, $K$. Lane and U. Mahanta, Phys. Rev. Lett. 61, 1553 (1988).

6. T. Apprelquist, T. Takeuchi, M. B. Einhorn, L. C. R. Wijewardhana, Phys. Lett. B220, 223 (1989): T. Takeuchi, Phys. Rev. D40, 2697 (1989); V. A. Miransky and K. Yamauraki. Mod. Phys. Lett. A4, 129 (1989).

7. R. S. Chirukula, A. G. Cohen and K. Lane, Nucl. Phys. B343, 554 (1990).

8. E. Eichten, K. Lane and J. Preskill, Phys. Rev. Lett. 45, 225 (1980).

9. J. Preskill, S. Trevedi, F. Wilezek and M. B. Wise, Nucl. Phys. B363, 20 T (1991); L. Krauss and S. -J. Rey, Yale Preprint YCTP-P9-92, to appear in Physical Review Letters.

10. R. Sundrurn, preprint LBL-32107 and UCB-PTH-92/08; L. Randall, MIT Preprint, in preparation.

11. E. Eichten, 1. Hinchliffe, K. Lane and C. Quigg, Rev. Mod. Phys. 56, 579 (1984); Phys. Rev. D34, 1547 (1986).

12. K. Lane and E. Eichten, Phys. Lett. B222, 274 (1989).

13. K. Lane and M. V. Ramana, Phys. Rev. D44, 2678 (1991).

14. J. Bagger, S. Dawson and G. Valencia, Phys. Rev. Lett. 67, 2256 (1931). 
15. R. S. Chivukula, M. Golden and M. V. Ramana, Phys. Rev. Lett. 68, 2883 (1992).

16. R. Cahn and M. Suzuki, Phys. Rev. Lett. 67, 167 (1991).

17. R. S. Chivukula and M. Golden, Nucl. Phys. B372, 44 (1992).

18. R. S. Chivukula and M. Golden, Phys. Lett. B267, 233 (1992).

19. R. S. Chivukula, M. Golden and M. V. Ramana, Boston University preprint BUHEP92-23 and Phys. Lett. B, in press.

20. R. S. Chivukula, M. Golden, and M. Dugan, Boston University preprint BUHEP-9218.

21. M. Soldate and R. Sundrum, Nucl. Phys. B340, 1 (1990).

22. S. Dimopoulos, B. W. Lynn, S. Selipsky and N. Tetradis, Phys. Lett. B253, 237 (1991).

23. C. Ahn, B. Lynn, M. Peskin and S. B. Selipsky, Nucl. Phys. B309, 221 (1988).

24. B. W. Lynn, S. B. Selipsky, R. G. Stuart and D. Levinthal, Boston university preprint BUHEP-90-36(rev); in preparation.

25. D. DeCamp, et al., ALEPH Collaboration, preprint CERN-PPE/91-105, submitted to Z. Phy's. C.

20. M. Golden and L. Randall, Nucl. Phys. B361, 3 (1991).

27. R. S. Chivukula, M. Golden, and M. Dugan, Boston University preprint BUHEP-9225.

28. M. Peskin and T. Takeuchi, "Estimation of Oblique Electroweak Corrections," SLACPLB.5618 (Nov. 1991); R. N. Cahn and M. Suzuki, Phys. Rev. D.44,3641 (1991).

29. A. Longhitano, Phys. Rev. D. 22, 1166 (1980) and Nucl. Phys. B188,118 (1981); R. Renken and M. Peskin, Nucl. Phys. B211, 93 (1983); B. Holdom and J. Ternirig, Physs. Lett. B247, 88 (1990); A. Dobado, D. Espriu, and M. J. Herrero, Phys. Lett. B253, 405 (1990); H. Georgi, Nucl. Phys. B363, 301 (1991).

30. R. S. Chivukula, E. H. Simmons, and S. B. Selipsky, Phys. Rev. Lett. 69, 575 (1992).

31. A. G. Cohen, in preparation.

32. M. Golden and B. Hill, Phys. Lett. B254, 225 (1991).

33. M. Dugan, M. Golden and B. Grinstein, On the Hilbert Srace of the Heavy Quark Effective Theory, Phys. Lett. b282, 142 (1992).

34. An Expression of Interest to Construct a Major SSC Detector, submitted to the Superconducting Super Collider Laboratory, (July 1, 1991).

35. GEM Letter of Intent, The GEM Collmboration, submitted to the Superconducting Super Collider Laboratory, (November 30, 1991).

36. R. S. Chivukula, Proceedings of the 1988 DPF Summer Study on High Energy Physics in the 1990s, Snowmass, CO, June 27-July 15, 1988.

37. E. Eichten, K. Lane and M. Peskin, Phys. Rev. Lett. 50, 811, (1983).

38. R. S. Chivukula and L. Randall, Nucl. Phys. B326, 1 (1989). 
39. R. S. Chivukula, M. Golden and E. H. Simmons, Phys. Lett. B257, 403 (1991).

40. R. S. Chivukula, M. Golden and E. H. Simmons, Nucl. Phys. B363, 83 (1991).

41. Responses to the December 1991 PAC Report, The GEM Collaboration, GEM-TN-131 (July 8, 1992).

42. GEM Baseline I, GEM-TN-76 (April 23, 1992); Status of GEM Baselines, GEM-TN130 (July 10, 1992).

43. O. Eboli, R. Jackiw and S.-Y. Pi, Phys. Rev. D37, 3557 (1988).

44. M. Samiullah, O. Eboli and S.-Y. Pi, Phys. Rev. D44, 2335 (1991).

45. G. F. Mazenko, Phys. Rev. Lett. 54, 2163 (1985); Phys. Rev. D34, 2223 (1986).

46. A. G. Cohen, in preparation.

47. L. Dolan and R. Jackiw, Phys. Rev. D9, 3320 (1974); S. Weinberg, ibid D9, 3357 (1974).

48. A. Sakharov, Zh. Eksp. Teor. Piz. Pis'ma 5, 32 (1967).

49. S. Dimopoulos and L. Susskind, Phys. Rev. D18, 4500 (1978); M. Yoshimura, Phys. Rev. Lett. 41, 281 (1978); (E) 42, 746 (1978); D. Toussaint, S. Treiman, F. Wilczek and A. Zee, Phys. Rev. D19, 1036 (1979).

50. G. 't Hooft, Phys. Rev. Lett. 37, 8 (1976).

51. V. Kuzmin, V. Rubakov and M. Shaposhnikov, Phys. Lett. B155, 36 (1985); P. Arnold and L. McLerran, Phys. Rev. D36, 581 (1987).

52. A. Cohen and D. Kaplan, Phys. Lett. B199, 251 (1987); Nucl. Phys. B308, 913 (1288).

53. A. Cohen, D. Kaplan and A. Nelson, Phys. Lett. B245, 561 (1990).

54. A. Cohen, D. Kaplan and A. Nelson, Nucl. Phys. B349, 727 (1991); Phys. Lett. B263, $80(1991)$.

55. A. Colien and A. Manohar, Phys. Lett. B265, 406 (1991).

56. R. S. Chivukula and T. P. Walker, Nucl. Phys. B329, 445 (1990).

5j. A. De Rujula, S. L. Glashow and U. Sarid, Nucl. Phys. B333, 173 (1990).

58. R. S. Chivukula, A. Cohen, S. Dimopoulos and T. Walker, Phys. Rev. Lett. 65, 957 (1990).

59. S. Barr, E. Farhi, and R. S. Chivulula, Phys. Lett. B241, 387 (1990).

60. A. Cohen and D. Kaplan, Phys. Lett. B215, 67 (1989).

61. S. Bahrall, B. W. Lynn and S. B. Selipsky, Nucl. Phys. B325, 606 (1989).

62. S. Bahcall, B. W. Lynn and S. B. Selipsky, Nucl. Phys. B331, 67 (1990).

63. Ser for example. F. Wilczek, "Lectures at the Ferrava School," preprint IAS-SNS. HE.P.89/59 (1989).

64. R. Jackiw and S.Y. Pi, Phys. Rev. Lett. 64, 2969 (1990).

65. R. Jackiw and S.Y. Pi, Phys. Rev. D42, 3500 (1990). 
66. J. Hong, Y. Kim and P.Y. Pac, Phys. Rev. Lett. 64, 2330 (1990): R. Jackiw and E. Weinberg, Phys. Rev. Lett. 64, 2334 (1990).

67. R. Jackiw, S.Y. Pi and E. Weinberg, p. 268 in the proceedings of Progress in High Energy Physics, W. Pauchy Hwang, S.-C. Lee, C.-E. Lee and D. Ernst, eds. (North Holland, Amsterdam, 1991).

68. R. Jackiw and S.Y. Pi, Phys. Rev. Lett. 67, 415 (1991); Z. Ezawa, M. Hotta and A. Iwizzaki, Phys. Rev. Lett. 67, 411 (1991).

69. R. Jackiw and S.Y. Pi, Phys. Rev. D44, 2524 (1991).

70. R. Jackiw and S.Y. $\mathrm{Pi}$, to be published in the proceedings of the Tenth Symposium on Theoretical Physics, Mt. Sorak, Korea, July 1991; Yukawa International Seminar, Kyoto, Japan, July/August 1991; IAMP Congress, Leipzig, Germany, July/August 1991.

71. G. Dunne, R. Jackiw, S.Y. Pi and C. Trugenberger, Phys. Rev. D43, 133? (1901).

72. M. Camperi. F. Levstein(MIT) and G. Zemba(MIT), Phys. Lett. 247B, 549 (1990).

73. E. Witten, Commun. Math. Phys.100, 197 (1985).

74. R. Rohm, Annals of Physics 189, 223 (1989).

75. R. Rohm and B. Grinstein, Comm. Math. Phys. 111, 667 (1987).

ic. R. Rohm. B. Grinstein and J. Preskill, Caltech preprint, to appear.

7i. M. Green and J. Schwarz, Phys. Lett. 149B, 117(1984).

78. R. Rohm. D. Gross, J. Harvey and E. Martinec, Phys. Rev. Lett. 54, 502 (1985);

R. Rohm. D. Gross, J. Harvey and E. Martinec, Nucl. Phys. B256, 253 (1985);

R. Rohm. D. Gross, J. Harvey and E. Martinec, Nucl. Phys. B267, 75 (1986).

70. R. Rohm. Nucl. Phys. B237, 553(1984).

80. L. Dixon. J. Harvey, C. Vafa and E. Witten, Nucl. Phys. B261, 678 (1985).

81. P. Ginsparg and C. Vafa, Nucl. Phys. B289, 414 (1987).

E2. R. Rohm, M. Dine, N. Seiberg and E. Witten, Phys. Lett. B156, 253 (1985).

83. R. Rohm and E. Witten, Annals of Physics 170, 454 (1986).

84. M. Dine and N. Seiberg. Phys. Lett. 162B, 299 (1985).

85. D. Gepner, Nucl. Phys. B296, 757 (1988).

80. M. Dine, P. Huet and N. Seiberg, Nucl. Phys. B322, 301 (1989); R. Rohm and D. Nemeschansky, "Corners in Calabi-Yau Moduli Space," unpublished.

87. A. Cohen, G. Moore, P. Nelson and J. Polchinski, Proceedings of the Workshop on Unified String Theory, ed. M. Green and D. Gross; Nucl. Phys. B167, 143 (1986); Nucl. Whyss. B281, 127 (1987).

88. G. Horowitz, J. Lykken, R. Rohm and A. Strominger, Phys. Rev. Lett. 57, 283 (1986).

89. G. Horowitz and A. Strominger, Phys. Lett.185B, 45 (1987).

90. A. Strominger, Phys Rev. Lett.58, 629 (1987). 
91. G. Horowitz, J. Morrow-Jones, S.P. Martin and R.P. Woodard, Phys. Rev. Lett.60, 261 (1988).

92. P. Fendley and P. Ginsparg, Nucl. Phys, B324, 549 (1989).

93. P. Fendley, J. Phys. A22, 4633 (1989).

94. L. Krauss and F. Wilczek, Phys. Rev. Lett. 92, 1221 (1989); K. Li, Nucl. Phys. B361, 437 (1991).

95. P. Fendley, S. Mathur, C. Vafa and N. Warner, Phys. Lett. B243, 257 (1990).

96. P. Fendley and K. Intriligator, Nucl. Phys. B372 553 (1992); "Scattering and Thermodynamics in Integrable N=2 Theories," BUHEP-92-5, HUTP-91-A067, to appear in Nucl. Phys. B

97. P. Fendley, W. Lerche, S.D. Mathur and N.P. Warner, Nucl. Phys. B348, 66 (1991).

98. P. Fendley, Phys. Lett. 250B 96 (199n).

9). C.N. Yang and C.P. Yang, J. Math. Phys. 10, 1115 (1969); Al.B. Zamolodchikov, Nucl. Phys. B342 695 (1990).

100. M. Creutz, L. Jacobs and C. Rebbi, Phys. Rev. Lett. 42, 1390 (1979).

101. M. Creutz, L. Jacobs and C. Rebbi, Phys. Rep. 95, 201 (1983).

102. Lattice Gauge Theories and Monte Carlo Simulations, C. Rebbi ed., World Scientific Publishing Co., Singapore (1983)

103. K. Wilson, Phyu. Rev, D10, 2445 (1974).

104. J. Potvin and C. Rebbi, Phys. Rev. Lett. 62, 3062 (1989).

10j. S. Huang. J. Potvin, C. Rebbi and S. Sanielevici, Phys. Rev. D42, 2864 (1990).

106. H. Gausterer, J. Potvin, C. Rebbi and S. Sanielevici, Boston Univ. preprint 92-16 (1092), submitted for publication.

10i. R. Brower, S. Huang, J. Potwin and C. Rebbi, Phys. Rev, D., to be published.

108. R. Brower, S. Huang. J. Potvin, C. Rebbi and J. Ross, Phys. Rev. D, to be published.

109. R. Browcr, C. Rebbi and E. Vicari, Phys. Rev. 43D, 1965 (1991).

110. R. Brower, C. Rebbi and E. Vicari, Phys. Rev. Lett. 66, 1263 (1991).

111. R. Brower, K. Moriarty, C. Rebbi and E. Vicari, Phys. Rev. D43, 1974 (1991).

112. R. Brower, R. Edwards, C. Rebbi and E. Vicari, Nucl. Phys. B336, 689 (1901).

113. R. Brower and P. Tamayo, Phys. Rev. Lett. 62, 1087 (1989) and Nucl. Phys. B9, $50 \mathrm{i}$ (1983).

114. R. Brower and S. Huang Phys. Rev. D41, 708 (1990) and Phys. Rev, to be published.

$115 \mathrm{~K}$. Moriarty, E. Myers and R. Strillka. Comp. Phys. Commun. 54, 273 (1080).

110. K. Moriarty, E. Myers and R. Strilks, Phys. Lett. B207, 411 (1088).

11\%. E. Myers, C. Rebbi and R. Strilka, Phys. Rev. D45, 1355 (1992).

118. R. Brower and M. Naunberg, Nucl. Phys. B180, 221 (1980); R. Brower, P. Rossi and C.-I. Tan. Phys. Rev. D4, 942 (1981) and Nucl. Phys. B180, 699 (1981).

119. R. Brower, N. Deo, S. Jain and C.-1. Tan, Harvard University preprint in preparation. 
120. S. Cecotti, P. Fendley, K. Intriligator and C. Vafa, "A New Supersymmetric Index," BUHEP-92-14, HUTP-92-A021, SISSA 68/92/EP, submitted to Nucl. Phys. B.

121. P. Fendley and $H$. Saleur, " $N=2$ Supersymmetry, Painleve III and Exact Scaling Functions in 2D Polymers," BUHEP-92-15, YCTP-P13-1992, subrnitted to Nucl. Phys. B.

122 A. Cohen, A. Nelson and D. Kaplan, "Debye Screening and Weak Scale Baryogenesis," UCSD/PTH 92-19, BUHEP-92-20, submitted to Phrs. Lett. 


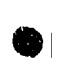

-

-

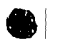

-

-

-

i

.

: 
TASK F - MUON G-2 EXPERIMENT AT BNL
Faculty:
Professor J. P. Miller (NSF)
Professor B. L. Roberts (NSF)
Professor L. R. Sulak
Assistant Professor W. Worstell (P.I.)
$\begin{array}{ll}\text { Research Faculty: } & \text { R. Carey } \\ \text { Graduate Students: } & \text { D. Brown, M. Rothman }\end{array}$
Undergraduate Students: S. Doulas, S. Korjanevski, X. Zhang
Collaborating Institutions: Brookhaven National Laboratory, CUNY, Fairfield, Heidelberg,
Institute for Nuclear Physics (Novosibirsk),
I.os Alamos, KEK, Tokyo, Yale

\section{Project Overview:}

The AGS ES21 precision measurement of the muon anomalous magnetic moment (g*2) will be sensitive to the virtual production of massive particles at the $\mathrm{TeV}$ energy scale, thus providing a sensitive test for physics beyond the standard model. In a succession of three experiments at CERN before 1976, the muon $\mathrm{g}-2$ value was measured to a precision of 7.3 ppm. The CERN measurements established the muon as a point-like lepton obeying QED with additional corrections from virtual pions, and provided constraints on the virtual production of new particles with masses on the order of tens of GeV. By increasing the precision of this measurement by a factor of 20 , we will measure the radiative contribution from $\mathrm{W}$ and $\mathrm{Z}$ bosons to within $20 \%$ of its standard model value, and thus test electroweak renormalization.

This measurement is sensitive to "new physics" such as muon substructure to several TeV, supersymmetric particles, an anomalous W-boson magnetic moment, a CP-violating muon electric dipole moment, and other physics beyond the standard model (Table 1). In several physics channels the sensitivity of E821 to "new physics" goes well beyond that which will be available at FNAL, LEPII, or the SSC. 
Table I

Sensitivity to New Physics of the E821 Muon $(g-2)$ Experiment

$\begin{array}{cc}\text { New Physics } & \text { E821 Sensitivity } \\ \mu \text { substructure } & \Lambda \geq 5 \mathrm{TeV} \\ \text { excited muon } & m_{\mu^{*}} \geq 400 \mathrm{GeV} \\ W^{ \pm} \text {substructure } & \Lambda \geq 2 \mathrm{TeV} \\ \frac{(g w-2)}{2} \neq 0 & \geq 0.02 \\ \text { Light Higgs } & \leq 300 \mathrm{MeV} \\ \text { Heavy Higgs } & \leq 500 \mathrm{GeV} \\ \text { Supersymmetry } & \leq 130 \mathrm{GeV} \\ W_{R}^{ \pm} & \leq 250 \mathrm{GeV} \\ Z^{\prime}(E 6) & \leq 100 \mathrm{GeV}\end{array}$

Cornments

SSC domain

LEPII comparable

LEPII $\sim 100-200 \mathrm{GeV}$

LEPII, SSC $\sim 0.2$

$O\left(10^{-3} \mathrm{~g}\right)$ coupling

$O(g)$ coupling

FNAL p̄p collider .

FNAL $\bar{p} p$ collider

FNAL $\bar{p} p$ collider

${ }^{a}$ For substructure $\Delta a_{\mu} \sim m_{\mu}^{2} / \Lambda^{2}$

In order to search for new physics in the several $\mathrm{TeV}$ range, we must first ascertain the value of the muon $\mathrm{g}-2$ which we should expect from the standard model. The dominant :ontribution comes from QED and is very precisely known, since QED correctly predicts the electron anomalous magnetic moment $(\mathrm{g}-2)$ to a level several orders of magnitude better than is nerded for the QED contribution to the muon $\mathrm{g}-2$. Because the muon is much more massive than the electron, Feynman diagrams with massive particles (quark loops, muons, taus, and weak bosons) contribute measurably to the final result. The relative contribution of heavier particles to the unuon's anomalous mornent goes as $\sim\left(m_{\mu} / m_{c}\right)^{2}$ which makes the contribution of such particles to the muon anomaly much larger $\left(\sim 4 \times 10^{4}\right)$ than to the electron anomaly.

Although QED and electroweak contributions to the muon $g-2$ have been precisely calculated (to 0.05 and $0.09 \mathrm{ppm}$, respectively), the contribution from the virtual production of hadrons has not. This contribution, however, may be related through dispersion theory to the experimentally measured cross-section for $e^{+} e^{-}$annihilation into hadrons. The overall contribution of virtual hadrons to the anomaly is $60 \mathrm{ppm}$, with a current uncertainty of about $1.5 \mathrm{ppm}$. This is of the same order as that of the weak boson contribution $(1.8 \mathrm{ppm})$, so that although there is a window of opportunity for new physics in going from the CERN $7.3 \mathrm{ppm}$ measurement to a $1 \mathrm{ppm}$ measurement, we will need an improved value 
for the hadronic contribution to $g-2$ in order to achieve our full physics reach $(0.35 \mathrm{ppm})$. For this reason, we are collaborating closely with a group at the Institute for Nuclear Physics (Novosibirsk) in a plan to measure hadron production over the $\rho$ resonance (up to $\sqrt{s}=1.4 \mathrm{GeV}$ ) to the necessary accuracy. Their improved storage ring (VEPP.2M) is now operational, as is a new detector (CMD-2). Data collection with CMD-2 began in early 1992, and with data taken in 1992-1993 CMD-2 should be able to reduce the uncertainty

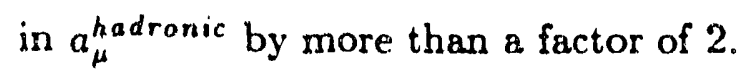

In order to improve on the $1.5 \mathrm{ppm}$ systematic errors present in the final CERN measuement of the muon $\mathrm{g}-2$, we have made substantial improvements in all areas of the experiment. A much more uniform storage ring magnetic field will be provided by a monolithic superferric magnet currently under construction, which will feature both a continuous yoke and continuous pole pieces. We will have the capability of injecting either pions or muons into the storage ring with a DC superconducting inflector, avoiding the transient kicker fields experienced at CERN. The magnetic field will be continuously monitored with NMR probes, and the entire field will be periodically mapped by an NMR trolley which will travel inside the storage ring without having to turn off the magnet or remove the vacuum chamber. The vacuum beampipe will have a "scalloped" geometry designed to eliminate electron pre-showering in the vacuum chamber walls. Finally, we have introduced $\mathrm{n}$ number of improvements in detector and data acquisition systems, including monitoring of the phase space distribution of the stored muons as a function of time. Storage ring completion is anticipated in 1993 and data collection will commence in late 1994.

\section{Progress in Muon g-2 Detector System Development}

The primary responsibility of the Boston University DoE Task F group has been evolving within the E821 collaboration. Previously, we had been constructing scintillating . fiber EM calorimeters and their associated lightguides, along with PMTs, bases, and data acquisition electronics. With the addition of the Illinois NSF group to the E821 collab. oration, we are shifting our focus downstream of the EM calorimeter (starting with the lightguides, and still including PMTs, bases, and DAQ electronics). This includes the task of designing and constructing the precision timing electronics in collaboration with electronics engineers from BNL and BU. We successfully constructed and tested scintillating fiber EM calorimeter prototypes for our Spring 1992 BNL beamtest, but future responsi- 
bility for scintillating fiber calorimeter construction is expected to be taken by the Illinois group. In accordance with our new emphasis, we have constructed high-rate blanking PMT bases and a prototype waveform digitizer module in association with the MACRO experiment.

The g-2 experiment involves the measurement of the arrival times of electrons with energies above a given threshold. In the context of pion injection into the storage ring, these precise timing measurements must be made a few tens of microseconds after the "flash" of scintillation light generated by electromagnetic debris striking calorimeters at injection time (from the hadronic showers of pions which do not decay in flight). The extremely small systematic errors which we require imply that rate-dependent timing shifts from pile-up and other effects must be constrained to less than 30 picoseconds on average over a 1 millisecond observation time. These requirements determine the specifications of the PMTs and their bases, and of the data acquisition electronics and calibration system.

\section{Detector System Design Simulations}

In 1992, the Boston University group released several updates of our detailed storage ring and detector system Monte Carlo simulation program, G2GEANT. Stored muons are input from an upstream program which contains a full pion injection simulation with betatron oscillations, pion interaction losses, and decays in flight. Next the muons are propagated until their decay, after which time decay electrons (and/or their shower secondaries) are tracked through the magnetic field until they strike a calorimeter or exit from the storage ring. The program provides the user with the option of a very detailed GEANT simulation of the storage ring geometry, including vacuum beampipe, focusing quadrupoles, and storage ring magnets.

Although G2GEANT was developed on the VAX, we have ported the code to a number of other platforms, including Suns, SGIs, and most recently, an HP workstation at the University of Illinois. We have taken full advantage of the GEANT graphics capabilities, both in code development and in data analysis. Since last summer, the electron traceback system, the focusing and kicker electrodes, the trolley rails, as well as their various support pieces, were included in the simulation. We used G2GEANT to study a number of detector issues: the performance of the proposed decay-electron traceback system, the resolution of our scintillating fiber calorimeters, and the question of the electrodes' effect on the PSD and calorimeter. 
In modeling the traceback system, many of our design criteria were straightforward. We used low- $\mathrm{Z}$ materials, aluminum and Mylar, gave the chambers a healthy separation of $35 \mathrm{~cm}$ to make the sagitta large, and placed the first wire chamber as close as we possibly could to the storage region in order to minimize the distance over which we performed the traceback. In the course of our studies we learned about several other important considerations. First of all, we discovered a strong radial bias in the acceptance of the wire chambers. Specifically, electrons born at the inside of the storage region are more likely to strike all three wire chambers than those born on the inside. We developed a simple geometric correction to handle this problem. We also found that it was very important to make an average correction for the energy lost by particles in passing through the the chamber walls. This loss of energy means a smaller radius of curvature and a traceback radius which is systematically low.

To assess the performance of the systern, we compared the mean field and pitch corrections made first with the true muon distribution and then with our traceback distributions. In both cases the traceback corrections were quite good. We cannot make the electric field correction with our traceback distribution alone, for that depends on the equilibrium radii of the muons while the traceback calculates their instantaneous positions. There are still a few more studies to be completed, but the g-2 note on our simulation analyses is now bring used by our colleagues at the University of Illinois to help them in the design of a real systern.

The other detector studies were more modest in scope. We tested the resolution of our calorimeters with an ensemble of electrons from muon decays. Making fiducial volume cuts (kerping the electrons more than $1 \mathrm{~cm}$ from any edge) we obtain a resolution of $13.5 \%$ over the square root of the energy, slightly larger than a straight test-beam style simulation would give. The effect of the electrodes on the calorimeter is negligible. Even for the . thickest possible electrodes, the only effect on the energy response is a slight tail. The fit resolution is unchanged. We also used the electrodes in a rather unorthodox fashion. By thickening them to $3 \mathrm{~mm}$ (aluminum) and letting thern cover the full inside of the ring, we were able to mock up the vacuum tank arrangement of the CERN III experiment. In this case, the calorimeter performance was significantly degraded ( 23 percent over the square root of the energy), demonstrating the importance of the new, scalloped design. Studies of the electrodes' effect on the PSD are nearly complete. 


\section{Prototype Detector System Construction and Beam Tests}

At Boston University, we have built a succession of prototype scintillating fiber EM calorimeters; these devices were constructed from plastic optical fibers embedded within low melting-point bismuth/lead/tin/cadmium alloy. The $70^{\circ} \mathrm{C}$ melting point of the eutectic alloy is well below the softening point for the plastic fiber or its cladding. Electron bearn tests were made of cast EM prototypes in the A2 beamline at the BNL AGS during June 1992. Two scintillating fiber calorimeter prototypes were tested for a variety of beam energies, incident positions, and incident angles. These devices contained $3 / 4 \mathrm{~mm}$ diameter scintillating fibers in a $30 \%$ packing fraction, resulting in a $1 \mathrm{~cm}$ aggregate radiation length. Electrons with energies of $1-5 \mathrm{GeV}$ were used to study the light yield, uniformity, resolution, and pulse shape from these devices. Clear Lucite light guides were used to carry optical signals from full-size g-2 prototype calorimeter modules to 2" PMTs (3 PMTs per module). Mirrors were attached to the end of the modules after casting and machining to increase uniformity of response for different incident positions along the fiber axes.

Figure 1 indicates the response of these devices to $3 \mathrm{GeV}$ electrons (the low-energy tail was a consequence of other devices being tested up-beam). The light yield was more than $900 \mathrm{PEs} / \mathrm{GeV}$ before filtering; after filtering to remove short wavelength (short at tenuation leng(h) light. the yield was still greater than $450 \mathrm{PEs} / \mathrm{GeV}$. Photoelectron statistics make a negligible contribution to the resolution. By using filters, we obtained a uniform response to within $5 \%$ when the incident position varied along the length of the fbers. This permits "radial readout" of the fiber calorimeters, which considerably simplifies our light guide design. The detector resolution was given by the combined contribution of a $7 \%$ energyindependent term plus a $\frac{10 \%}{\sqrt{E}}$ stochastic term, with the two terms adding in quadrature. The latter term is in good agreement with GEANT predictions, while the former term is apparently caused by variations in the optical coupling to the mirrors on the fiber ends. Although the devices we tested met all g-2 specifications for resolution, uniformity, light yicld, and compactness, we do not currently plan to produce the electron calorimeters for the $\mathrm{g}$. 2 experiment. In the interests of the larger collaboration, we are concentrating our future efforts on parts of the detector system which do not as yet have working tested prototypes - the PMT, base, and front-end DAQ electronics. 


\section{PMT, Base, and Data Acquisition Electronics}

Our current schedule for the muon g-2 experiment calls for design and development of data acquisition electronics to be completed by mid-1993. High-speed electronic systems which are extremely insensitive to rate-dependent effects are at the heart of this precision experiment. It is essential that we move beyond the present conceptual design stage to development and construction of a full-scale prototype. Lead times for electronics design, and the benefits of at least a two-generation prototype approach, are such that a vigorous effort in this area during the coming year is very important to the future of the entire project.

We have designed and constructed a high-rate gated PMT base for $\mathrm{g}-2$, and have tested it in association with a Hamamatsu R1828 photomultiplier. A schematic for this device is included as Figure 2. Preliminary indications are that it can switch the PMT off in less than 10 microseconds, and return the PMT to more than $95 \%$ of its original gain in less than 10 microseconds after being switched back on. It can also produce consistent $100 \mathrm{mV}$ pulses (within 5\%) in response to LED flashes varying in frequency from DC to $10 \mathrm{MHz}$. We have also designed and are constructing a random pulse generator (or LED driver) with a $20 \mathrm{MHz}$ instantaneous peak rate and an exponentially falling average rate (with a 64.4 microsecond decay constant). This device will be used to trst the rate dependence of both photomultiplier tubes and their associated downstream data acquisition electronics with a more representative pulse train than can be provided by a normal pulser.

During the past year, we have done some preliminary electronics design in coordination with waveform-digitizer (WFD) development for the MACRO experiment. Many of the attributes of a $200 \mathrm{Megasample/second} \mathrm{zero-suppressing} \mathrm{waveform} \mathrm{digitizer,} \mathrm{based} \mathrm{on} \mathrm{ECL}$ flash ADC technology, are directly relevant to g-2 electronics needs. This could be used as both a multihit $A D C$ and a pulse pile-up rejection circuit, and we will further develop this . design to address our specific needs during the coming year. Another BU device, which like the WFD is based on a BU-designed ASIC (Application Specific Integrated Circuit), is a $1 \mathrm{GHZ}$ multihit TDC (MTDC) being developed for the DUMAND experiment. This device appears very well-suited to our readout requirements for a wire chamber traceback system, for a scintillator veto/pileup rejection system, and especially for a segmented PSD.

When combined with a precision "de-randomizing" circuit, either the WFD or the multihit TDC can serve as a "digitron" for the g-2 measurement. The "de-randomizing" 
circuit unambiguously associates asynchronous discriminator outputs with a synchronous precision clock, and is under development at BNL. A "digitron" here denotes a precision zero-suppressed multihit TDC with rate-dependent average timing shifts of no more than 10 picoseconds. The digitron forms the cornerstone for the rest of the data acquisition electronics system, and while we have assembled some prototypes of its components, it will require quite a bit more design and testing. Further elements of our design include a low-drift $125 \mathrm{MHz}$ temperature-controlled precision clock and a proposed optical clock signal distribution system. The first prototype WFD module is now in the BU EDF (Electronics Design Facility), and after it has been tested for MACRO we will measure its bi-phase performance in its $\mathrm{g}-2$ configuration.

\section{Collaboration with CMD.2 Experiment at Novosibirsk.}

As part of our ongoing collaboration with the CMD-2 experiment, we have produced a multianode PMT-based scintillating fiber PSD for use with a "beam strahlung" photon luminosity monitor. The high-rate high-gain multianode PMT base we developed for this purpose may have additional applications for reading out a $g-2$ scintillating fiber PSD. We have also developed electronics for hardware data-reduction and integration with the CAMAC-based CMD-2 data acquisition system. The entire system was tested at the BNL A3 test beam, in ussociation with SSCintCAL test bean work, with typical set of pulse height distributions corresponding to a $1 \mathrm{~cm}$ beam spot (in coincidence with an upstream "finger" counter) given in Figure 3. The device has a position resolution of $2 \mathrm{~mm}$ and an efficiency per plane of better than $70 \%$; this efficiency has a geometrical origin and is inconsequential for the CMD-2 application. In the very near future, a Boston University graduate student (D. Brown) plans to convey the BU PSD to the Institute for Nuclear Physics in Novosibirsk, install it in CMD.2, and use it to contribute to a more accurate mensurement of the VEPP-2M collider luminosity.

In anticipation of a CMD-2 analysis effort at Boston University, we have installed the latest CMD-2 simulation and offline analysis codes on the BU VAX cluster; we are in the process of porting this software to our UNIX platforms. We have received an $8 \mathrm{~mm}$ tape of raw data and are beginning to implement a data reduction sequence. Our primary physics interest in the CMD-2 results is the measurement of the hadronic vacuum polarization and its contribution to the $8-2$, but we are also exploring other physics channels in order to evaluate systematic errors in measuring the machine's luminosity and acceptance. 


\section{Future Plans:}

Although we will continue to be involved in electron calorimeter optics and mechanical support, we will concentrate our future efforts on parts of the detector system which do not as yet have working tested prototypes - the PMT, base, and front-end DAQ electronics. We will be guided in our detector system design by continued detailed simulations of detector response and its consequences for the g-2 measurement. The detector system goal is to produce a full detector station (calorimeter, lightguides, pile-up rejection scintillator hodoscopes, PMTs, bases, much of the front-end electronics, and mechanical support structure) in time for Spring 1993 BNL beam testing.

\section{Detector System Design Simulations}

We anticipate an increasing number of software development projects as the experimental hardware becomes more and more well-defined. We already have a substantial simulation framework in place, both in terms of GEANT particle generation and propagation and in terms of preliminary "data analysis" software. Intermediate between these we have detailed optical Monte Carlo and detector response programs with which we can match the performance measured in bench and beam tests. The G2GEANT framework will thus require continuous upgrading of both its "upstream" interface with the E821 accelerator physics group input and its "downstream" interface with the representation of realistic detector system response.

Still further "downstream", software developed to test and read out VME-based data acquisition electronics (discussed below) may well form the groundwork for the E821 "frontline" and online software systems. Last but certainly not least, we have begun to synthesize the effects of possible systematic errors into effects on the anticipated muon-decay electron arrival distribution; this effort forms the first threads of an offline analysis effort. To this end, a set of programs have been developed which first produce simulated spectra with known systematic errors, then fit various waveforms for the precession frequency. The performance of each of the detector systems, as ascertained with the calibration system and with real data (particularly data incorporating wire chamber or PSD information) must be characterized in terms that lead directly to systematic error corrections. By performing software analyses of the anticipated magnitude of these effect.s, we take the first steps toward assessing and sometimes correcting for them in the real experiment. The organization 
and systematization of all these efforts will be a very substantial undertaking for which we must plan and work well in advance of actual data-taking. In parallel with this effort, we plan to further strengthen our collaboration with the CMD-2 experiment, which will be collecting the data most relevant to the hadronic vacuum polarization determination within the coming year.

\section{Prototype Detector System Construction and Beam Tests}

By June 1993, the Boston University g-2 group, working with the Illinois group, will have produced a final full-size prototype electron calorimeter station. Boston contributions will include light guides, high-rate gated PMTs, an interface with the detector calibration system, and a largely complete set of prototype data acquisition electronics. 'The prototype station will then be tested in a BNL AGS test beam and the results used to confirm our overall detector system design. Between July 1993 and June 1994, we will begin production of light guides, PMT bases, and data acquisition electronics. Full detector system production must commence by July 1993, since calibration and installation are scheduled to begin in late 1993, so that detector systems can be operating within the storage ring by 1994. Our plan of attack is to work our way downstream from the calorimeter, first establishing good light collection and rate-independent PMT performance (through the use of prototype calibration systems) and continuing with measurement of the performance and stability of data acquisition electronics for both the calorimeter and the rest of an integrated detector system.

\section{PMT, Base, and Data Acquisition Electronics}

We propose to thoroughly test the rate dependence of our proposed PMT with its high-rate gated base, plus the DUMAND MTDC and the MACRO WFD, and to characterize the limits of their suitability for the muon $8-2$ experiment. We would provide the resources needed to construct $\mathrm{g}-2$ prototypes, where these resources are small compared to the overhead in ASIC and PCB development costs supported by these other projects. Since both prototypes are being constructed for VMIE environments, we will need to build up a laboratory VME interface as an initial prototype for the $\mathrm{g}-2$ data acquisition system. We will also need to acquire a precisely stable clock and a frequency synthesizer as part of a preliminary electronics calibration and testing system. Work on data acquisition and front-end electronics will be carried out in close coordination with the Instrumentation 
Group at BNL. We anticipate an evoluticaary development through a series of prototypes in order to meet the very dernanding rate-independence specifications of this experiment; it is essential that this progression commence as soon as possible in order to complete the requisite number of design cycles before we must freeze the instrumentation design by early 1994.

\section{Collaboration with CMD.2 Experiment at Novosibirsk}

The 1993 calendar year will be an important time for close coordination with the CMD-2 experiment at Novosibirsk; late 1992 and 1993 will include the longest data-taking phase - -levant to measuring the photoproduction of hadrons. This measurement is directly related to the $\mathrm{g}-2$ contribution from hadronic vacuum polarization, and is the only experiment which will have measured this contribution to our required precision. For our experiment to achieve its physics goal of accurately measuring the electroweak contribution to the muon $\mathrm{g}-2$ and probing $\mathrm{TeV}$ physics, it is essential that this contribution be accurately determined and that we have confidence in the results. For this reason, we have reguested funds for travel to Novosibirsk to participate in these measurements, and a small axwount of funding to support visits from our Novosibirsk collaborators to Boston Cuiversity. We have also requested a modest level of equipment funding in support of the CMID.2 experiment; our timely access to CMD-2 data (and its timely collection) are current!y contingent upon the availability of limited computer resources there. Disk space and $8 \mathrm{~mm}$ tape drives are essential for the collection and distribution of the high volume of data gencrated by the CMD-2 detector.

\section{Long Range Plans and Schedule}

The ES21 precision measurement of the muon anomalous magnetic moment is currently schertuled to begin taking data in early 1995. There may well be the possibility . of intermittent operation in "parasite" mode throughout some of the 1994 AGS running. We plan :o stage the experiment, running first (1995) with pion injection and then (1996) with muon injection. Direct muon injection will eliminate any problems with hadronic barkigrounds created by interacting pions within the storage ring, and will significantly increase the intensity of the stored muon bearn. It should also introduce significantly different systematic errors in the experimental measurement, allowing a double-check on our understanding of these errors. It is quite likely that we then would continue with a final 
run in 1997, either with changed beam polarity (if this has not been accomplished earlier) or with some other significant alteration or upgrade. In any event, it is safe to say that the final analysis of this very complex and demanding experiment will carry us into 1997 and beyond.

In terms of hardware responsibilities, we should have completed the bulk of our calorimeter construction within 1993 and have moved into testing and characterization in 1994. The calibration, readout, and data acquisition system development must be carried out in parallel with this effort. As our hardware efforts are completed our software and operational efforts promise to ramp up, with our base of operations progressively shifting to the experimental site by 1994. During the actual running of the experiment we anticipate our peak operations requirements, when we will also provide the peak learning experiences for young physicists and students. 


\section{References}

1. J. Bailey et al., Nucl. Phys. B150, 1 (1979).

2. Brookhaven National Laboratory Experiment E821, A New Precision Measurement of the Muon (g-2) Value at Level of $0.95 \mathrm{ppm}, \mathrm{V}$. W. Hughes, W. M. Morse, and B. L. Roberts, Spokesmen.

3. T. Kinoshita and W. J. Marciano, Quantum Electrodynamics, (Directions in High Energy Physics, Vol. 7), 'T. Kinoshita ed., World Scientific, 1990, p. 419.

4. T. Kinoshita, Quanturn Electrodynarnics, (Directions in High Energy Physics, Vol. 7) T. Kinoshita ed., World Scientific, 1990, p .

5. T. Kinoshita, B. Nižić and X. Okamoto, Phys. Rev. D31, 2108 (1985).

6. L. M. Barkov et al, Nucl. Phys. B256, 365 (1985).

7. J. A. Casas, C. López and F. J. Yndurán, Phys. Rev. D32, 736 (1985).

8. H. Burkhardt, F. Jegerlehner, G. Penso, C. Verzegnassi, Z. Phys. C43, 497 (1989).

9. Lubomir Martinovič and Stanislav Duvnička, Phys. Rev. D42, 884 (1990). 
Figure 1 Measured response of casr lead/fiber calorimeter to

$3 \mathrm{GeV}$ electrons in BNL A2 less beam. The low-energy

tail is a consequence of other devices being lested up-beam.
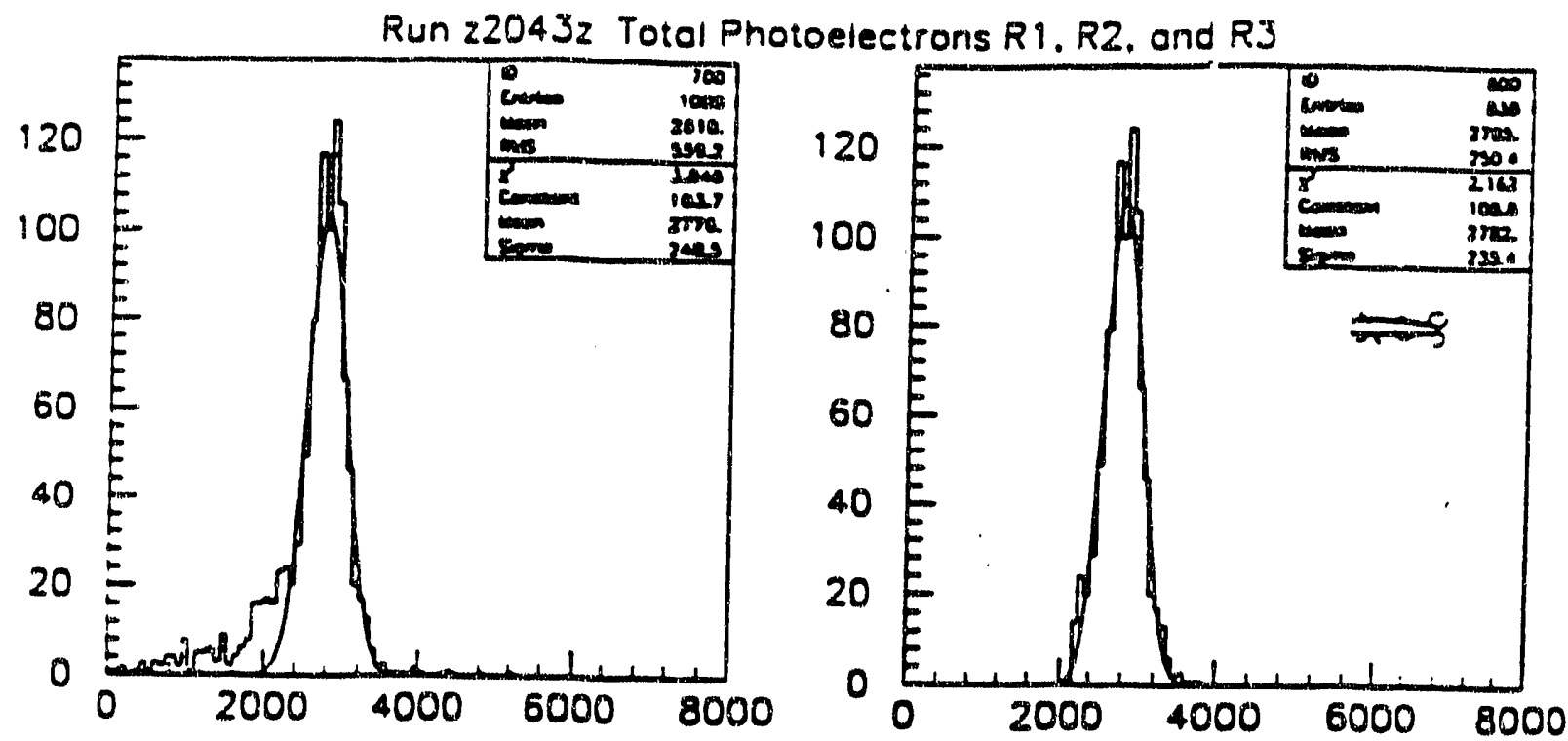

Digitol Sum

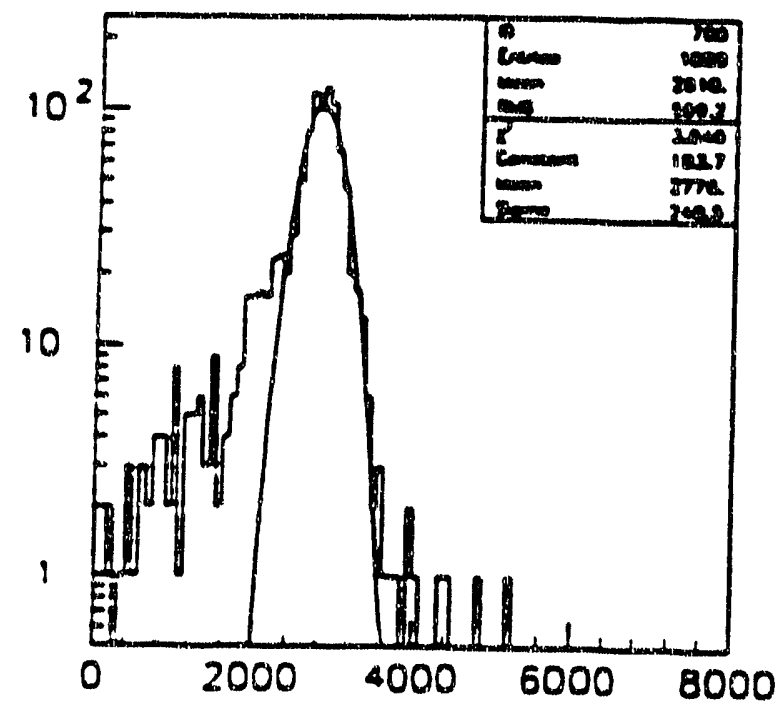

Digitol Sum
Digital Sum Cropped

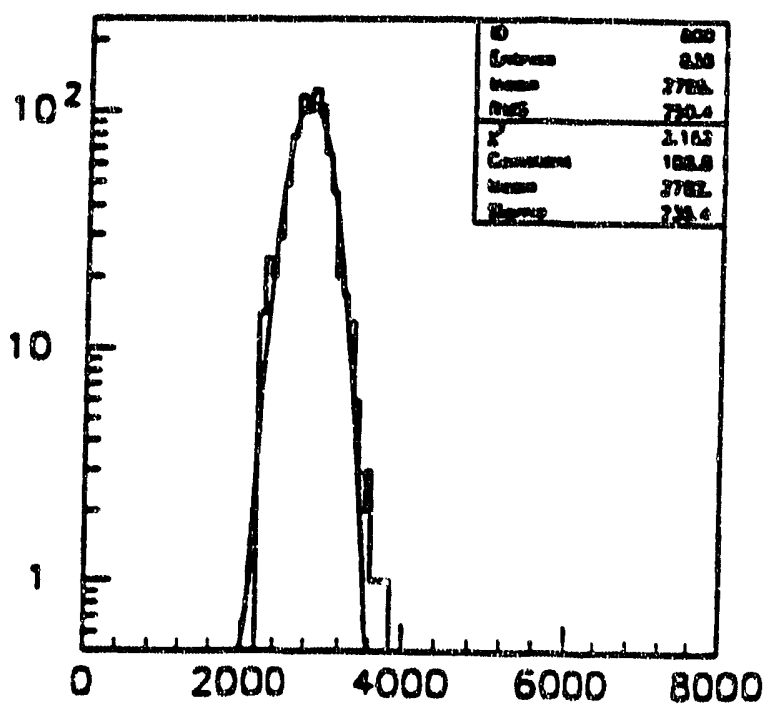

Digitat Sum Cropped

$9: 7$ PE/GeV

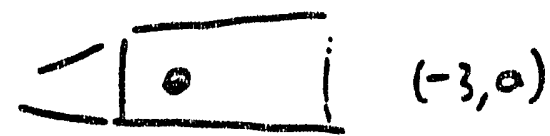


Figure 2 Electroaics schemaic for a high-rate, guted photomultiplier hase for a Hememats R1828 pholomultiplier. This device has been constructod and is being lesed to g-2 specifications.

$\frac{1}{2}$
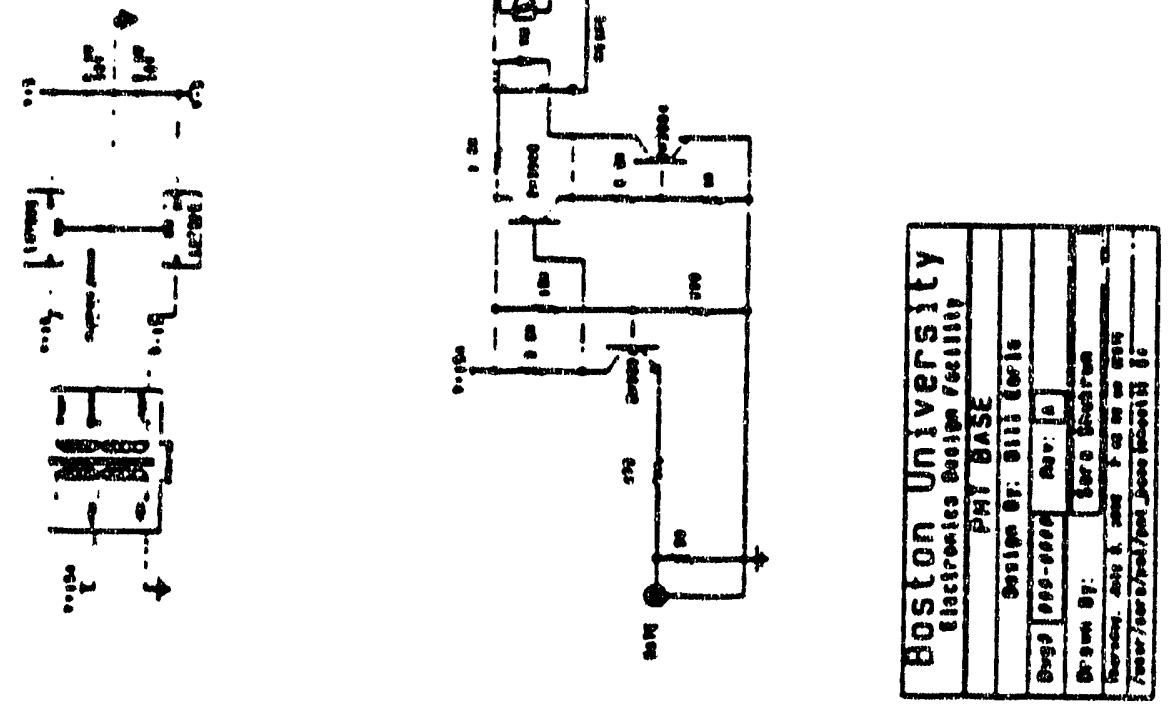

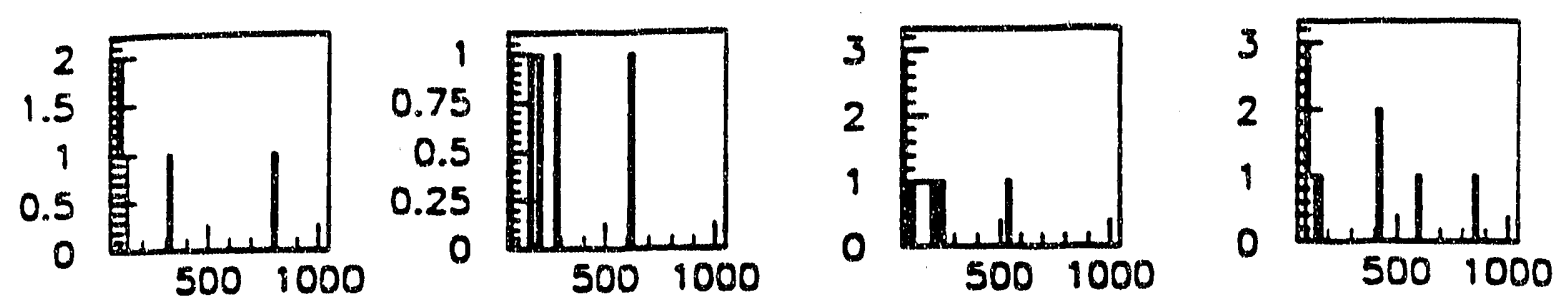

LMPSD Chan I ADC LMPSD Chon 2 ADC

LMPSO Chon 3 ADC LMPSD Chon 4 AOC
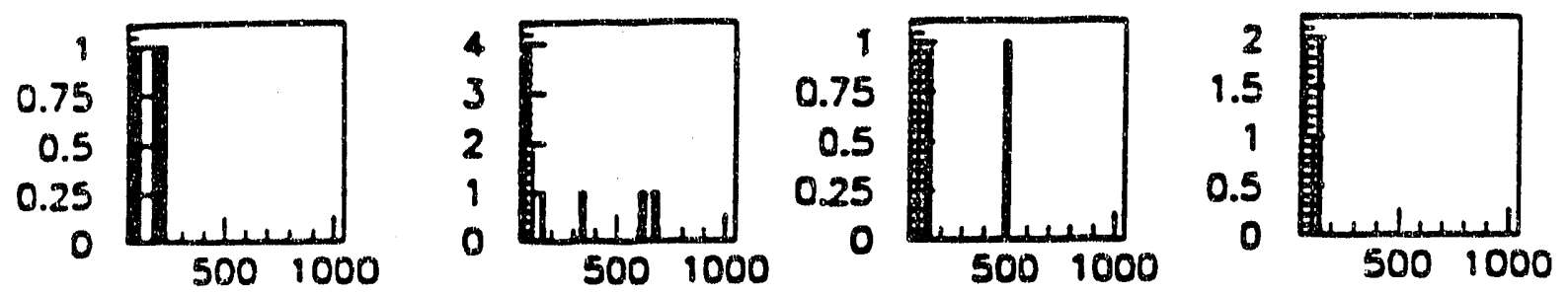

LMPSO Chan 5 AOC
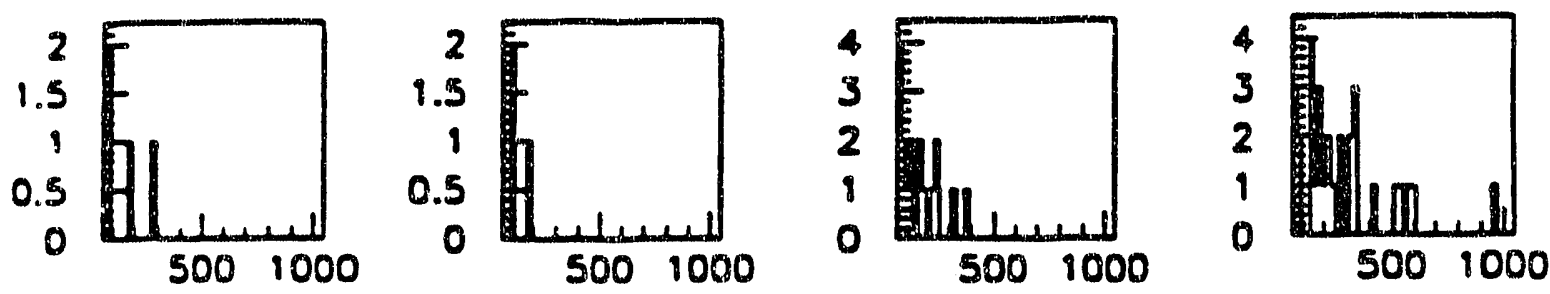

LMPSO CNON 9 ADC

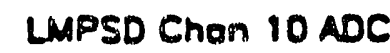

LUPSO Chan 11 ADC

LMPSD Chon 12 ADC
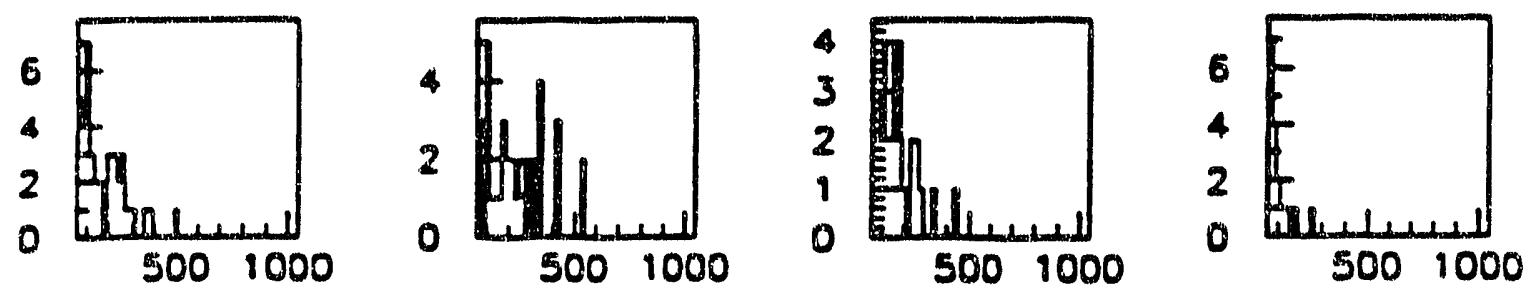

LAPSO Chon 13 NDC LUPSO Chon 14 ADC LMPSD Chon 15 ADC LMPSD Chon 16 ADC

Figure 3 Response (ADC diseribuioess) for 16 channels of scincillating Tiber hodoscope read ou Urough a 64-chennel multimode PMT.

The irciden paricles are $10 \mathrm{GeV}$ smons in the BNL. $\mathrm{A} 3$ vest beam. 


\title{
TASK J: TOPICS IN PARTICLE PHYSICS
}

\author{
Faculty: Assistant Professor Andrew G. Cohen \\ Graduate Student: $\quad$ S. Myint
}

\section{Research Accomplishments}

During the past few years Cohen's research has focused on questions of the electroweak phase transition and baryogenesis, with occasional forays into other areas. Much of this work is described in task $\mathrm{E}$ of this proposal. A brief summary is given here, with attention given to recent and pending developments.

In early studies of baryogenesis, Cohen and D. Kaplan suggested a scheme known as "spontaneous baryogenesis" in which the time dependence of scalar fields during a phase transition favors the production of a baryon asymmetry [1]. The effective Hamiltonian for fermions in the presence of these fields splits the energy levels of quarks and anti-quarks (or leptons and anti-leptons), and if the system contains baryon violating interactions, this will cause the population of the lower energy levels to be enhanced, thus producing the baryon asymmatry. The size of this asymmetry is set by these splittings, which is in turn set by the size of the variation of the scalar fields. If the phase transition is second order, this will be set by the Hubble parameter and a large asymmetry can only be generated at a relatively high temperature. If the transition is not second order, than the variation of the scalar field, and the associated baryon asymmetry, can be much larger.

Mlost models of electroweak baryogenesis use this scheme with a first order (or spin. odal) weak phase transition. In this case the varying scalar fields are typically Higgs fields (or any generic scalar field which develops a vacuum expectation value at the weak transition) which produces bubbles at this transition. Cohen, Kaplan and Nelson have computed the baryon asymmetry according to this scheme in a number of different models, including throries with multiple Higgs doublets, and a model of neutrino masses [2]. They found that the observed asymmetry could be easily accommodated provided that new $C P$ violation was sufficiently large. These models had the great advantage over other proposals in that they have experimental consequences, such as a large $\tau$ neutrino mass, electric dipole moments for the neutron or the electron, etc. 
Recently Cohen, Kaplan and Nelson have looked at finite temperature plasma effects on these models [3]. They have shown that the long range gauge forces and Debye screening have a negligible effect on baryogenesis. This result is essentially a consequence of anomaly cancellation-if anomalies cancel, then the value of any gauge charge does not affect the rate of anomalous processes. Thus even though gauge charges are screened, this does not alter the bias in anomalous processes.

Nelson and Cohen are completing a detailed study of the minimal supersymmetric standard model. In this model, baryogenesis is severely constrained; the gauginos must be relatively light in order to be available in significant numbers at the weak phase transition, and must not be too light to agree with current experimental bounds. In addition the new $\mathrm{CP}$ violation must be relatively large in order to explain the large observed baryon asymmetry. Thus all parameters are tightly constrained, and the large CP violation leads to a relatively large electric dipole moment for the neutron; in fact, the baryon asymmetry may already be in conflict with the neutron dipole moment in this model. These constraints may be relaxed by extending the model, notably through the addition of a singlet. This weakens the constraints for obtaining a first order transition, and therefore relaxes the constraints on several parameters. Thus this non-minimal model may provide a more favorable environment for baryogenesis.

Cohen, Kaplan and Nelson are currently writing a review of the subject of electroweak bryogenesis for Annual Reviews in Nuclear and Particle Physics. Such a review is quite timely considering the advances made in the last two years.

Cohen is currently working with $\mathrm{E}$. Weinberg and S. Coleman on a new technique for computing nucleation rates in first order phase transitions. Weinberg has recently shown that conventional techniques are inadequate to deal with false vacuum decay when this vacuum instability is the result of radiative corrections. These authors are considering the generalization of this technique to finite temperature. The conventional formalism relies on a perturbative computation of the free energy; this leads to an artificial imaginary part for the free energy, which when properly interpreted through an appropriate analytic continuation represents the lifetime of the unstable thermal state. However, when the transition is induced by radiative corrections this formula does not obviously apply, and these authors are considering this problem more carefully. The result could prove interesting, since the electroweak transition is passibly driven first order due to radiative corrections. 
Cohen is currently extending work on rare kaon decays. Previously he showed that several of these decays are dominated by non-analytic terms in chiral perturbation theory, and thus can be calculated independently of unknown coefficients in the chiral Lagrangian. With G. Ecker, Cohen is improving this calculation to the greatest extent possible, incorporating all corrections required by unitarity. There remain incalculable corrections (consistent with unitarity but not required by it) but the previous arguments by Cohen indicate that these corrections are quite small, on the order of a few percent. The result of this calculation changes the previous predictions by twenty to thirty percent.

\section{REFERENCES}

1. A. Cohen and D. Kaplan, Phys. Lett. 199B, 251 (1987); A. Cohen and D. Kaplan, Nucl. Phys. 308, 913 (1988).

2. A. Cohen, D. Kaplan and A. Nelson, Phys. Lett. B245, 561 (1990); A. Cohen, D. Kaplan and A. Nelson, Nucl. Phys. B349, 727 (1991); A. Cohen, D. Kaplan and A. Nelson, Phys. Lett. B265, 406 (1991); A. Cohen, D. Kaplan and A. Nelson, Nucl. Phys. B373, 453 (1992); A. Cohen, Baryogenesis at the Electroweak Phase Transition. Proceedings of the Hiroshima International Workshop on Electroweak Symmetry Breaking); A. Cohen, Baryogenesis and Phase Transitions, Proceedings of the $27 \mathrm{th}$ Rexcontres de Moriond, Electroweak Interactions and Unified Theories.

3. A. Cohen. D. Kaplan and A. Nelson, Debye Screening and Baryogenesis During the Electroweak Phase Transition, BUHEP-92-20. 
TASK K: SCINTILLATING FIBER CALORIMETRY FOR THE GEM DETECTOR AT THE SSC

Faculty:

Research Faculty:

Undergraduate Students:

Associated Faculty:

Collaborating Institutions:
Professor L. R. Sulak (P.I.)

Assistant Professor W. Worstell (co-P.I.)

R. Carey, S. Dye, J. Sullivan

E. Fellinger, C. Lin, A. Meyer,

T. von Rosenvinge, G. Stidham, K. Yoshida

Associate Professor J. P. Miller (NSF)

Professor B. L. Roberts (NSF)

Drexel, Fairfield, Iowa, Louisiana State, Michigan State, Mississippi, Mississippi State, Tennessee, Texas A\&M, Texas Tech, UC-San Diego

National Laboratory Affliates: C. S. Draper Lab, Oak Ridge, Lawrence Livermore

\section{Project Summary}

The Boston University SSCintCal group has taken the lead role in the construction of a 5-ton copper/scintillating fiber hadron calorimeter for the GEM SSC detector. After fabrication and assembly in the Boston University Scientific Instrument Facility (SIF), the device was tested at the BNL A3 test beam in July, 1992. Preliminary analysis of the , test results indicates good resolution, uniformity, and compensation for this new type of hadron calorimeter. Further analysis of these beam test results should allow us to optimize price/performance trade-offs in the GEM system context. A final decision on the hadron calorimeter for GEM (between scintillating fiber and liquid argon alternatives) will be made by the GEM collaboration management in October, 1992. 
The primary advantages of scintillating fiber calorimetry for the SSC include its intrinsic speed of response, modularity, uniformity and hermeticity, ease of construction and maintenance, stability, simplicity, and safety. In addition, scintillating calorimeters in large detectors at hadron colliders have a record of proven performance. The equal response to electromagnetic and hadronic showers of scintillating calorimetry offers the potential for hadronic energy resolution surpassing that possible with any non-compensated calorimeter.

The high energies at the SSC are best measured by precision calorimetry, which, unlike magnetic momentum analysis, increases its accuracy with increasing particle energy. To exploit fully this high resolution potential, it is essential that the calorimeter be compensated and uniform in its response. A uniform device is insensitive to shower fluctuations or precise incident particle positions. Missing energy signatures require that the calorimeter be free of cracks and nonuniformities, and that it provide coverage out to high rapidity for hermetic containment.

By constructing a five-ton prototype in a short length of time, we have demonstrated the performance required for the GEM hadron calorimeter and have added a great deal of credibility to our construction cost estimates. In addition to prototype construction, we have contributed to system engineering, manufacturing design, detailed Monte Carlo simulation, physics performance analysis, radiation damage/lifetime assessment, photoconverter and data acquisition electronics design, calibration system design, and integration of the hadron calorimeter with other GEM subsystems. Finally, we have established a firm basis for international collaboration by coordinating detector design and construction planning with colleagues from Albania, Belarus, the People's Republic of China, Ecuador, India. and Russia. The relative simplicity of the copper/scintillating fiber hadron calorimeter construction makes it accessible for contributions from scientists and engineers in less developed countries, as is evidenced by the above list of collaborators (all of whom have provided Letters of Intent or Interlaboratory Collaborative Agreements).

\section{Progress in FY9?}

For copper scintillating fiber calorimetry, we have carried out the following tasks in FY92:

1. Construction and beam testing of 5 tons ( 7 modules) of fully projective full-scale copper/SF prototypes, including radiation-hard fibers, fiber-optic bundles coupled to 
light mixers and PMTs, and a calibration system including radioactive sources and laser-driven quartz optical fibers.

2. Design and initial construction of 4-module "supertowers" for possible beam testing at CERN in the fall of 1992 . These will be prototypical of the units which will be constructed in quantity (1000 supertowers) for GEM.

3. Development of a consistent and cost-effective manufacturing, assembly, structural, and systems engineering design of a copper/fiber hadron calorimeter for GEM, with engineering support from Boston University engineers and Oak Ridge National Laboratory.

4. Preparation of the hadronic fiber calorimetry section of the GEM LoI, and preparation for the GEM technical design report.

5. Establishment of international collaborations for construction of the GEM hadronic fiber calorimeter.

These efforts have been carried out as a coherent technological subgroup within GEM. San Diego has provided the characterization and quality assessment of the scintillating fibers (as well as their SPACAL work). Boston has designed and constructed the prototype towers, including the optical package. Michigan State and Iowa managed the BNL test beam work, assisted by collaborators from Mississippi and elsewhere. Oak Ridge worked on radiation damage assessment for plastic scintillating fibers and also assisted in test beam work. Fairfield and Tennessee provided the calibration system and a quartz forward calorimeter. Louisiana State, as a part of the GEM muon group, provides us with specifications of calorimeter requirements for compatibility with the muon system. Texas A \&: $M$ and Drexel have developed a liquid fiber forward calorimeter subsystem for GEM. This forward calorimeter exploits many of the same technologies we are using in the central fiber calorimeter.

\section{Beam Tests of Hadronic Prototypes}

In July 1992, we used BNL $e, \mu$, and $\pi$ test beams up to $20 \mathrm{GeV}$ to evaluate:

1) Stochastic term in the energy resolution for EM and hadron showers,

2) Uniformity across tower boundaries and within individual towers,

3) Compensation and $e / \pi$ vs. beam energy,

4) Position resolution and shower lateral profiles,

5) Pile-up and resolution degradation in high-rate beam conditions, 
6) Punch-through and variations in shower longitudinal profiles, and

7) Response to muons and catastrophic energy los : by muons.

Figure 1a illustrates the energy resolution in response to electrons for a typical tower, corresponding to $\frac{60 \%}{\sqrt{E}}$. Figure $1 \mathrm{~b}$ shows the result of Monte Carlo electron simulations, which agree with the data in resolution. This rather modest resolution is the result of the small $(2 \mathrm{~cm}$ ) Moliere radius in copper and our coarse (1 fiber every $8 \mathrm{~mm}, 2.25 \%$ plastic by volume) sampling. Although modest, this electromagnetic resolution is sufficient for our electromagnetic physics requirements within the hadron calorimeter; these include the measurement of electromagnetic leakage from an upstream EM calorimeter, and the measurement of catastrophic EM energy loss (e.g., bremsistrahlung) by muons. Having demonstrated agreement between our data and Monte Carlo simulations, we can now project the EM resolution improvement which would result, from finer fiber sampling, and can thus optimize our price/performance trade-off.

Figure 2a illustrates our measured snergy resolution in response to pions, corresponding to $\frac{90 \%}{\sqrt{E}}$ after leakage corrections. Figure $2 b$ shows the result of GEANT/Gheisha Monte Carlo pion simulations, which agree with the data in resolution after tuning a single parameter (the calorineter's $e / h$ response). This resolution corresponds to a $\frac{75 \%}{\sqrt{E}}$ resolution for jets. which is sufficient for our physics goals. As with the electromagnetic resolution, we can now use Monte Carlo simulations to extrapolate from our tested design to predict the performance of a more fine-grained device, and thereby optimize price/performance in our system context. Figures $3 a$ and $3 b$ illustrate the agreement between data and Monte Carlo predictions for the fraction of hadronic shower energy deposited within the central tower of our 7 -tower stack; this allows us to correct our measurements for lateral leakage by simulating a larger array than we were able to build and test.

Our hadronic calorimeter prototype's response to muons is still under study. Since most of our muon data was taken during parasitic running 1 meter downstream of a liquid argon EM calorimeter and dewar, accurate muon ID requires more detailed analysis. Most data was also taken with the fiber axes within the central tower pointing almost directly along the incident muon trajectories, which is a geometry we will avoid in our final system implementation by making our towers slightly non-projective. Muons incident nearly parallel to fibers can traverse the entire calorimetes: without depositing significant energy in the fibers, and we observed this in our beam test conditions. By matching the 
observed muon efficiency with Monte Carlo predictions, we will be able to extrapolate to our final system configuration with greater confidence.

\section{Copper/Fiber Calorimeter Plans for FY93}

The primary objective of our FY93 R\&D and engineering study is to fabricate a fully hadronic shower-containing set of scintillating fiber prototype towers embodying the latest GEM SSC detector calorimeter design. The prototypes will be characterized in beam tests employing high-energy hadronic showers, and will be used to assess alternative manufacturing techniques, to establish the mechanical integrity of our calorimeter system design, and to refine cost projections. Because of our experience in FY92 and the infrastructure we have developed in producing our first generation of copper/fiber towers, we are confident we can produce this large prototype in a timely and efficient manner, so as to provide information on the scintillating fiber hadronic calorimeter performance. This effort will consist of the following tasks:

- Development of manufacturing techniques for laminated copper/fiber towers.

- Study of the mechanical interfaces between towers and the support structure.

- Further development of the optical interfaces and photomultiplier tube readout system.

- Resolution of cable and fiber routing issues.

- Study of the geometry requirements for towers in adjacent nested rings within an assembly of prototype towers, and the design of fixtures for the assembly in accordance with this study.

- Comprehensive finite element modeling and analysis of the towers and their connection ring structural assembly to identify stresses, deflections, and any material deformation.

- Design and construction of full-scale prototype hadronic supertowers in a projective geometry at a median value of $\eta$ using results of the above studies and feedback from our July 1992 BNL beam tests.

- Beam tests of a fully shower-containing, 15-ton hadronic calorimeter assembly for resolution, $e / \pi$, etc., at CERN (as discussed in the next section). 
- Assessment of detailed cost estimates of the design developed above relative to alternative designs,

Throughout FY93, our efforts will focus upon developing a dernonstration of a credible, cost-effective, and high-performance hadron calorimeter for the GEM detector. Our two years of TNRLC support have given us a substantial start on this effort, and have provided us with the infrastructure and experience we will need to rapidly produce GEM prototype calorineter modules. These prototypes will be integrated into the overall GEM calorimeter and detector engineering design, and will reflect our design and construction experience throughout the past year. As in the past year, we expect further evolution in our detector design and manufacturing methods on the basis of our experience with new materials and techniques.

As our mechanical, manufacturing, and engineering design becomes more mature, we will progressively transfer this technology to industrial concerns and to our international collaborators. We have already established a network of links with domestic and international engineers, scientists, and laboratories, and we will engage these collaborators and affiliates in our manufacturing process whenever it is consistent with our timescale and budget. Ultimately, the calorimeter system manufacturing and cost projection must reflect the economies of scale which these collaborators will achieve, and by coordinating our prototype manufacturing with their investment in fixturing and process control we can obiain a much better projection of the quality and cost of a complete scintillating fiber hadronic calorimeter for the SSC.

\section{FYgq Test Beam Goals \& Other Studies}

Our next set of beam tests will be performed upon 15 Tons of copper/fiber hadron calorimeter prototypes (or on 5 Tons of our current prototypes surrounded by several SPACAL towers to measure leakage):

Use of CERN $e, \mu$, and $\pi$ test beams up to $150 \mathrm{GeV}$ to evaluate:

1) Stochastic and constant terms in the energy resolution,

2) Uniformity across tower boundaries and within individual towers,

3) Compensation and $e / \pi$ vs. bearn exergy,

4) Position resolution and shower lateral profiles, 
5) Pile-up and resolution degradation in high-rate beam conditions,

6) Punch-through and variations in shower longitudinal profiles, and

7) Response to muons and catastrophic energy loss by muons.

In addition to the above beam testing, we anticipate:

Evaluation of effects of radiation exposure on calorimeter components and on full-scale prototype systems, including annealing and long-term stability studies; and

Test of mechanical stress and strain characteristics of prototype modules, and evaluation of sample cross-sections after beam tests are completed.

Also in a CERN test beam, we hope to conduct beam tests with a large array of $\mathrm{BaF}_{2}$ precision $\mathrm{EM}$ towers in front of our hadronic towers. Results from these tests will be used to develop and verify effective compensation strategies which allow for energy sharing between the EM and hadronic sections.

\section{Budget Discussion}

The present proposal requests purely operating funds from DOE/HEP for the management of our SSC subsystem task. These funds, along with the anticipated equipment support from the Texas National Research Laboratory Cornmission and the SSC Lab Subsystem R\&D program, will allow us to build a 15 -ton scintillating fiber hadronic calorimeter prototype for beam testing at CERN in the fall of 1992. This effort is being carried out as R\&D in support of the GEM detector for the SSC, for which the SSCintCAL device is one of two competing hadron calorimeter designs.

The B.U. SSCintCal group presently consists of four faculty (Miller, Roberts, Sulak, Worstell), two research associate professors (Dye, Sullivan), one research assistant professor . (Carcy), and six undergraduate research assistants (Fellinger, Lin, Meyer von Rosenvinge, Stidham, Yoshida). Principal investigators for this task are Prof. Lawrence Sulak and Asst. Prof. William Worstell. Prof. Sulak is a co-spokesman for the SSCintCal collabora. tion. which includes eleven university physics research groups and collaborators from three national laboratories. We also work closely with international affiliates as discussed earlier.

In this third year of our SSC subsystem effort, we are focusing on construction and testing of the 15-ton copper fiber hadronic calorimeter protokype. To provide experimental 
data necessary for GEM technical proposal decisions within the comirg year, it is essential to have the travel support request here to take advantage of test beams at CERN. These tests will be carried out in coordination with tests on a BaF electromagnetic calorimeter. Because there is little time available before the decision must be made for the technical proposal in the fall of '92, we must move forward vigorously and rapidly with prototype testing.

Our operations request represents primarily student, travel, and communications support for our SSCintCAL program. The GEM leadership has made it clear that GEM R\&D funds cannot be used to support communications and travel to the SSC Laboratory for collaboration and subsystem meetings. Neither do the anticipated TNRLC funds allow sufficient monies for transportation. However, these tasks are absolutely essential to the execution of our mission and the management of over $\$ 1 \mathrm{M}$ of equipment funds. Instead, it is expected that such support wil through the operating budgets of the individual institutions. Similarly, graduate siudents and postdoctoral fellows are expected to be established thro.dgh DoE HEP channels. Because of the scope of the responsibilities which we have issumed within SSCintCAL and GEM, the operations support which we have requested is vital both to us and to these collaborations. 


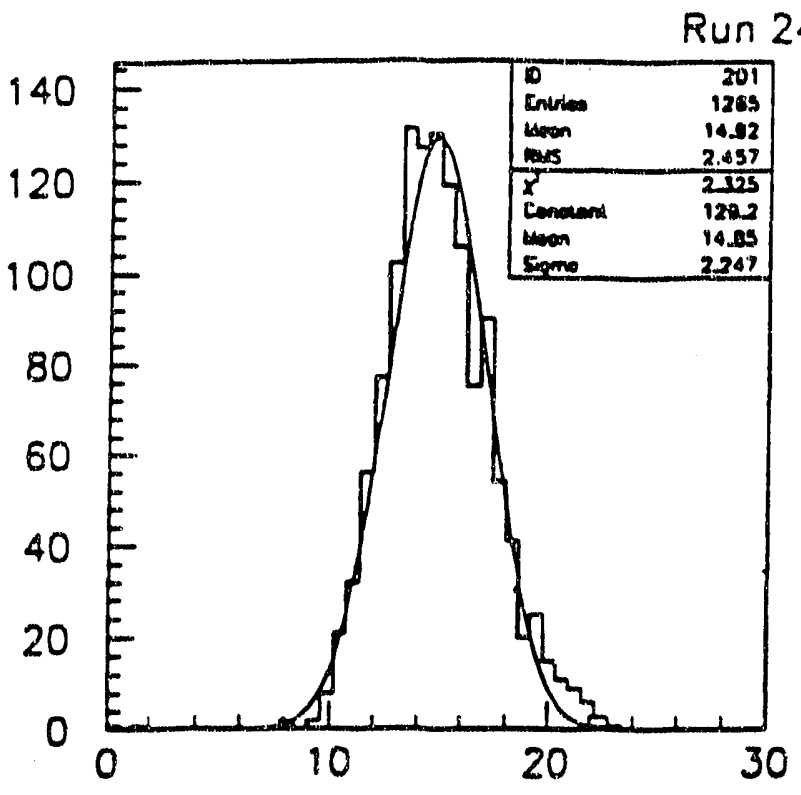

Module 2 signol in $\mathrm{GeV}$

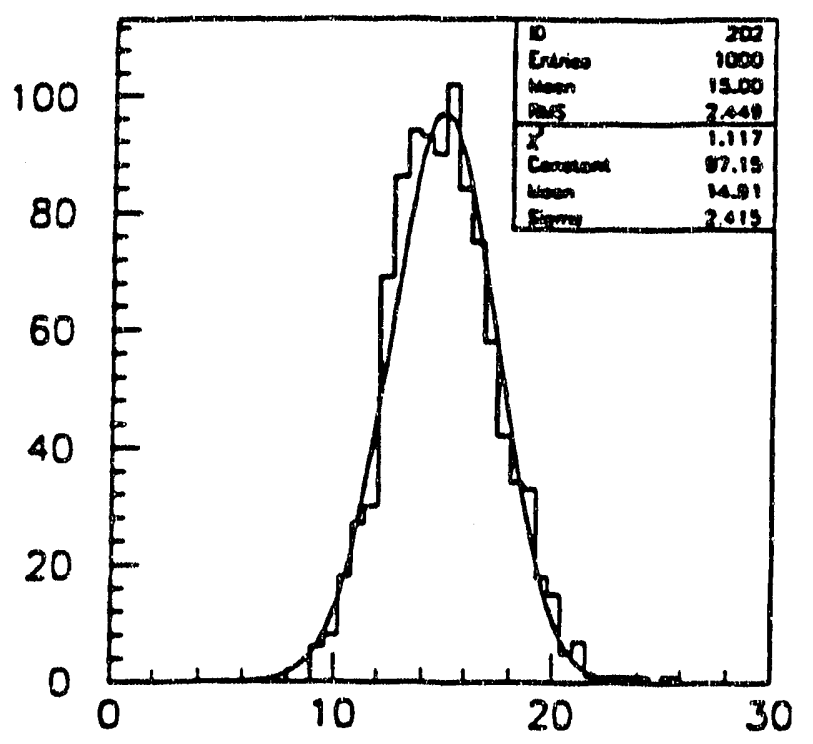

Simulation signal in GeV

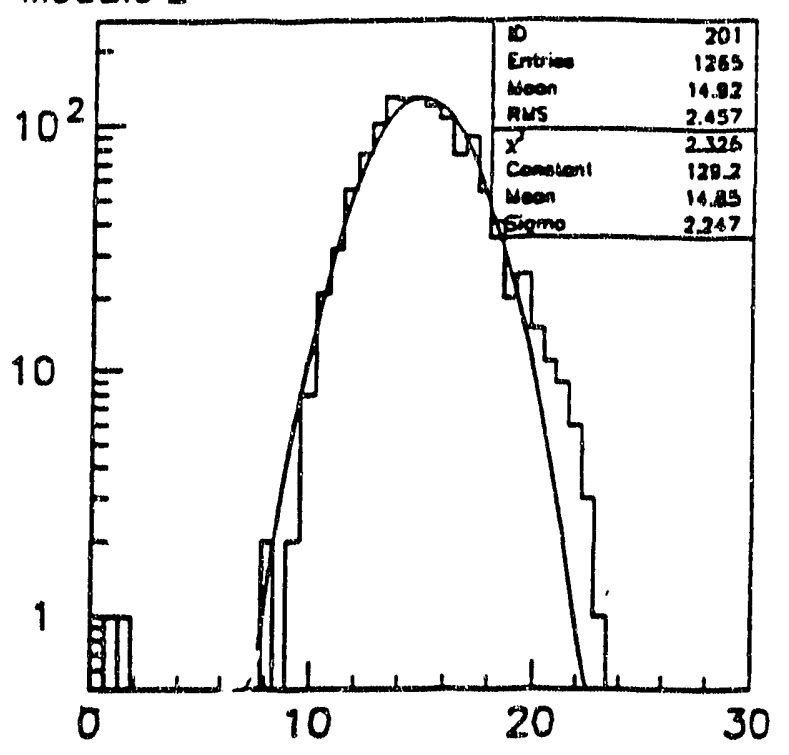

Module 2 signol in $\mathrm{GeV}$

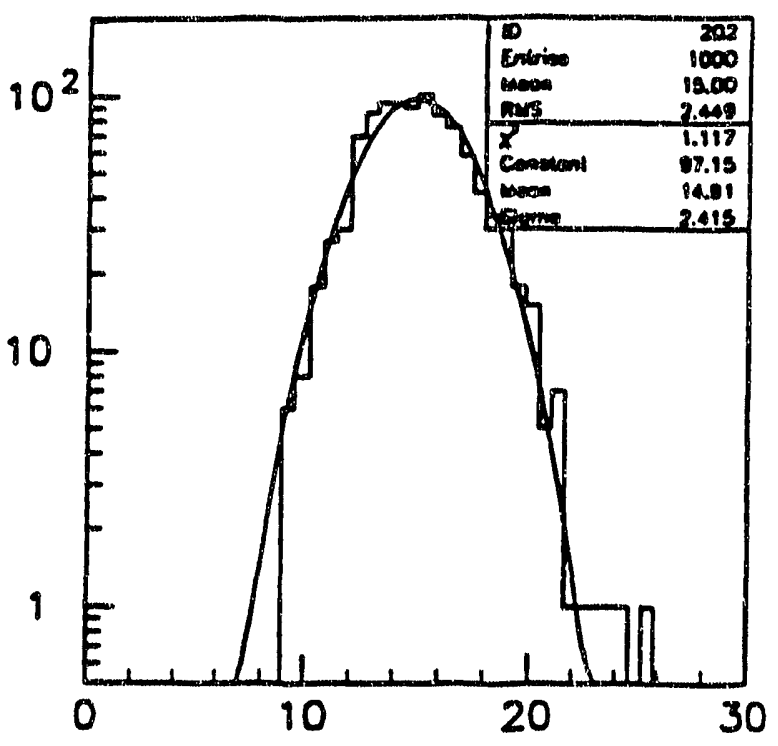

Simulation signal in $\mathrm{GeV}$

Fig 1 (a) Measured response of ecpper/tiber calorimeter

(top) 10 is GeV clectrons in BNL $A 3$ less beam.

Fig 1(b) GEANT simulared response wo $15 \mathrm{GeV}$ elocurous

(bottom) (left = linex, nightrefogarithmic display). 

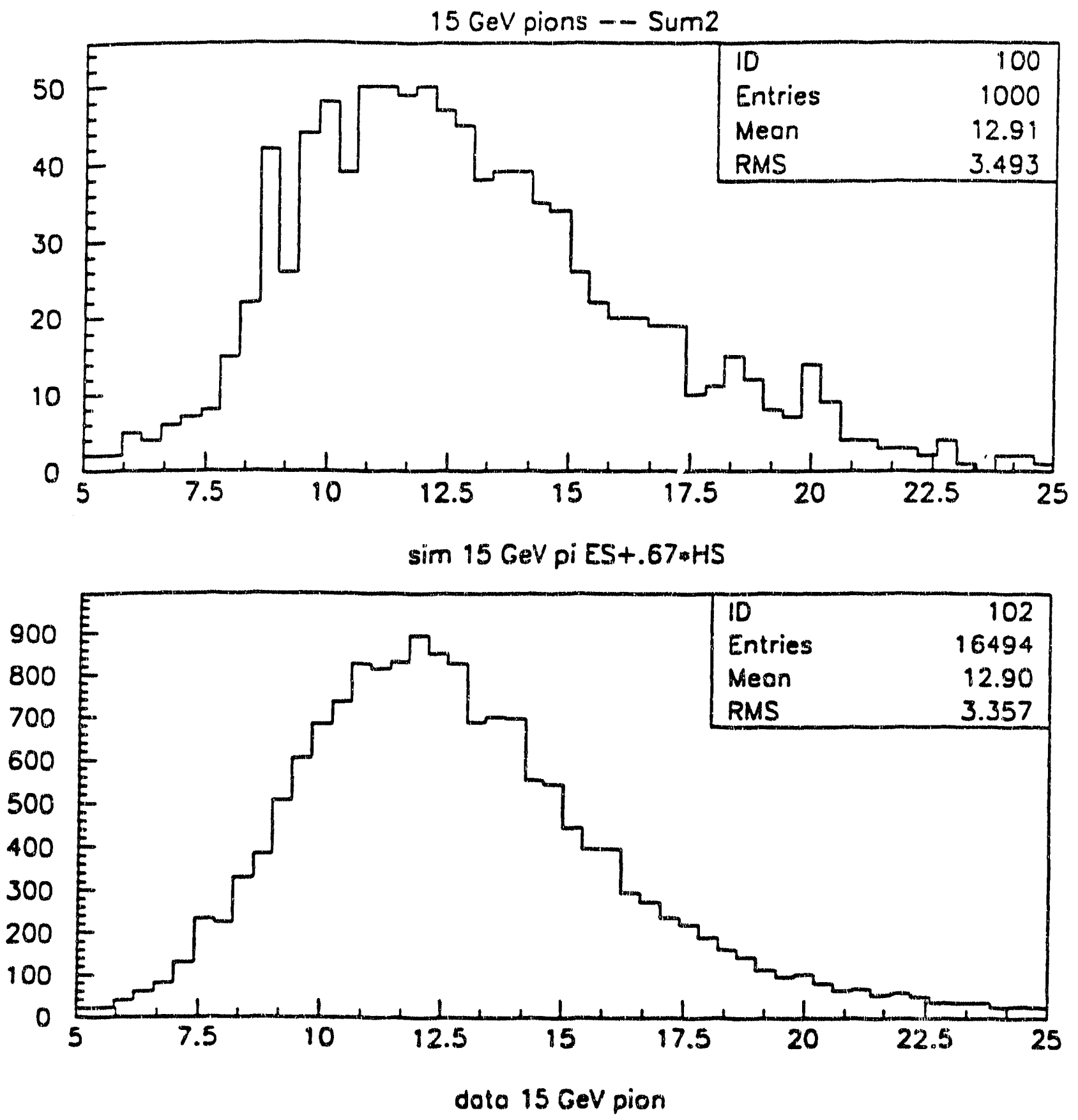

Fig 2(a) GEANT simulaced response to $15 \mathrm{GeV}$ pions (top) (ooe fuled perarseter a eth, no leakage corrections).

Fig 2(b) Measured response of copperfiber caloximeter vo (bottom) $15 \mathrm{GeV}$ pions in BNL A3 rest bearo (no leakenge corrections). 
$15 \mathrm{GeV}$ pions - - Sum2
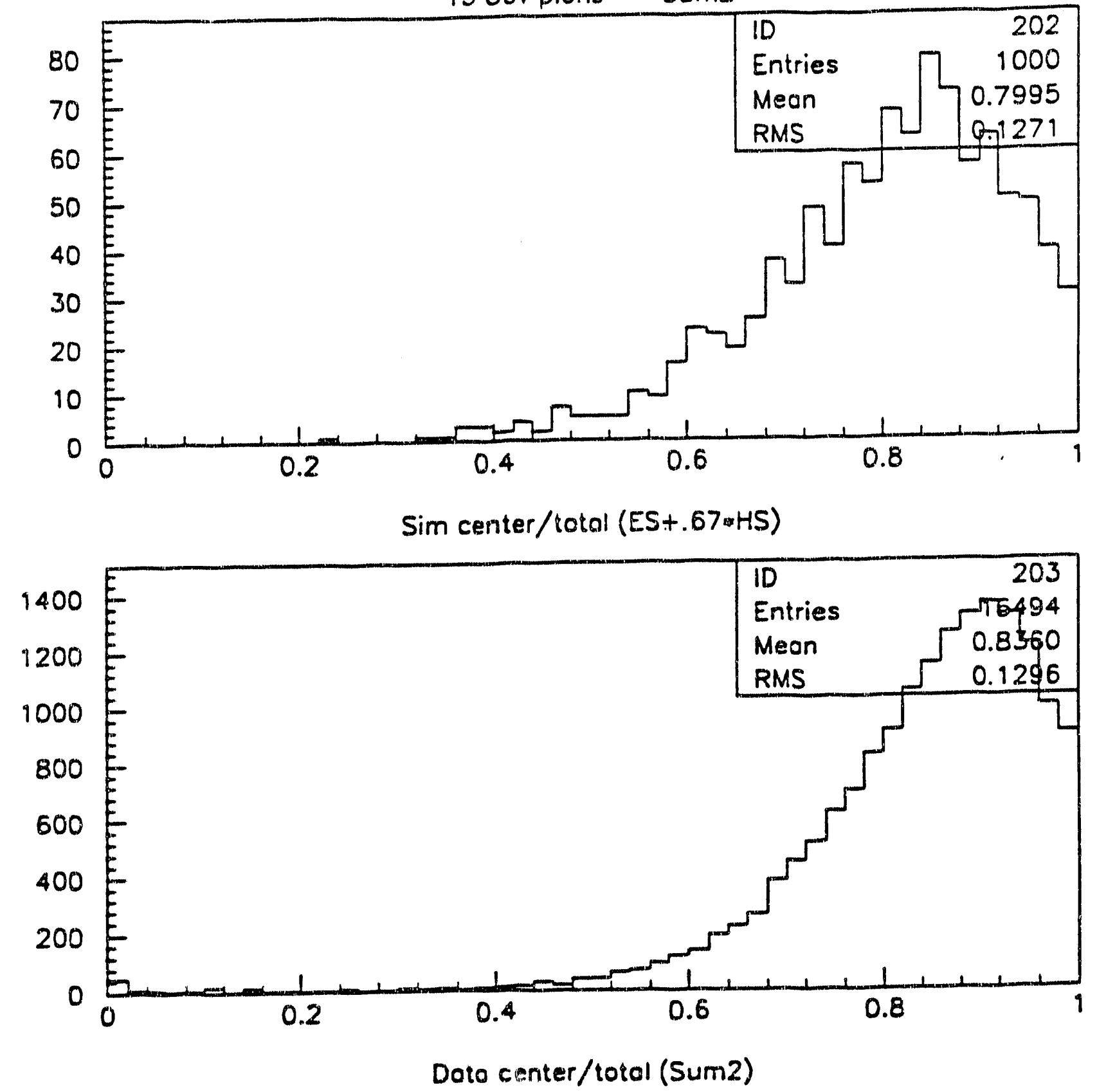

Fig 3(a) GEANT simulated firction of $15 \mathrm{GeV}$ pion shower (top) enengy within central motule of 7 -module arny (one fived parameter in esh).

Fig 3(b) Measured fracion of $15 \mathrm{GeV}$ pion showes energy (bottom) within ceurral module of 7-module array, for copper/inber module army in BNL A3 tex beam. 


\title{
TASK L: SSC MUON DETECTOR DEVELOPMENT
}

\author{
Faculty: $\quad$ Professor Scott Whitaker \\ Research Assistant Professor Anthony S. Johnson \\ Research Assistant Professor James T. Shank
}

Undergraduate Student: Jia Ming Chen

\section{Introduction}

Boston University researchers have been playing a major role in the GEM Experiment for the SSC. We are leading proponents in calorimetry and in muon systems and are prominent contributors to the physics analysis and simulations efforts for GEM. Task $L$ covers the development of muon detectors for the GEM endcaps $(1.3<\eta<2.5)$. NonSSC work of this group is covered in Task A. Support for this work has been provided by the TNRLC, as part of the GEM R\&D program, as well as by the DOE SSC Office. Two Boston University engineers, Gary Varner and Dave Warner, have been devoted full time to the electronics and mechanics, respectively. An undergraduate student, Jia Ming Chen. has also been very active in prototype detector construction. We are collaborating in this work with groups from Brookhaven National Laboratory, Oak Ridge National Laboratory, SUNX' Stony Brook, and the St. Petersburg Nuclear Physics Institute. Additional groups are interested in participating, and the project is developing an infrastructure and enginering support.

\section{Cathode Strip Chambers for the GEM Endcap Muon System}

The layout of the GEM detector is shown in figure 1. The module layout for the endcap is sketched in figure 2. Muon trajectories are measured in a 0.8 Tesla magnetic field outside the calorimeter. Muon momentum is determined by the sagitta method using measurements of muon position at the beginning, middle, and end of the particle's passage through the field region. 


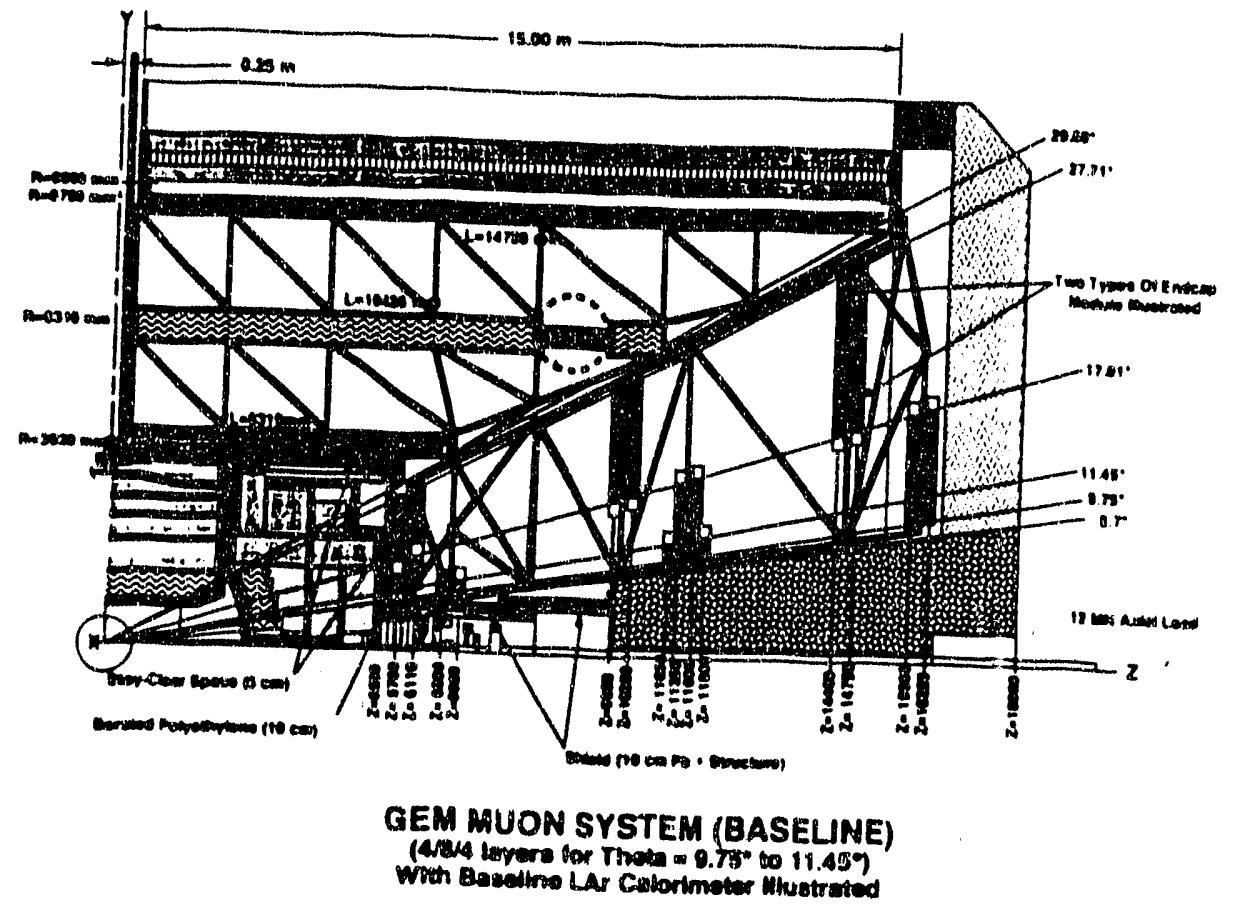

Fig 1 - Layout of the GEM Detector

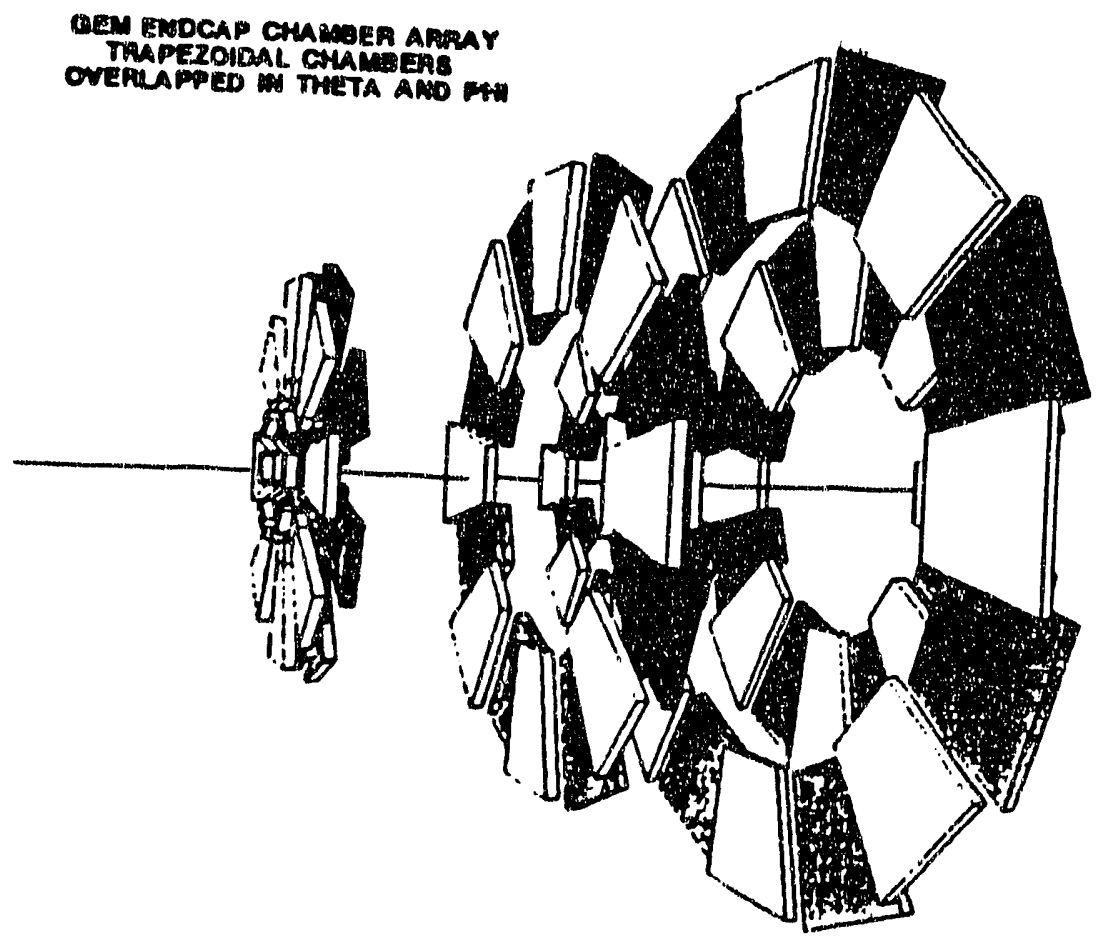

Fig 2. Cathode Strip Chamber module layout in the GEM endcaps. 
The endcap region will be covered by trapezoidal wire chambers with a small gas gap. and high-precision cathode strip readout. These Cathode Strip Chambers (CSC's) provide the full functionality of the muon system. Each module will consist of four chambers. The wires, which run in the azimuthal direction, are ganged for measurement of the radial coordinate with approximately $2 \mathrm{~cm}$ resolution. With a maximum drift time of approximately $20 \mathrm{~ns}$ in each chamber, a module will provide timing resolution of approximately $5 \mathrm{~ns}$ sufficient to tag the bunch crossing in which the interaction occurred. One of the cathodes in each chamber is segmented into radial strips from $20 \mathrm{~cm}$ to $3 \mathrm{~m}$ long, depending on the particle flux. Interpolation of the induced charges on the cathode strips will determine the azimuthal position of the track with better than $75 \mu$ resolution per chamber. Readout of the strips will be done with on-board multiplexing, so the cable plant will be minimal. Fast signals off the strips will be used to generate a first level trigger. The CSC modules overlap to provide complete coverage in the region between approximately 9.8 and 27.7 degrees in polar angle.

Test chambers and readout electronics have been constructed to study the time response, gain performance, and position resolution. Figure 3 shows a plan sketch and cross-section views of a prototype chamber under test at Boston University. The chamber has bern designed to be openable and so has only two gas gaps; two such chambers will be combined to form a module for measurement of position resolution. In the final design, chambers will be glued rather than bolted, and there will be four gas gaps per module. Measurement of the etched strip cathode has shown that the cathode features are accurate to 0.001 inches relative to the design dimensions over the roughly 20 inch square circuit board. Alignment features etched onto the strip cathode allow precise alignment of the chambers relative to an external coordinate system.

Readout circuits using the Amplex chip have been developed; equivalent noise charge of less than 2,000 electrons and channel-to-channel uniformity of better than $2 \%$ have been demonstrated. Custom integrated circuits are under development at BNL for the CSC's, and the Amplex solution is intended only for prototype tests. Nonetheless, the performance of the Amplex system is acceptable and engenders confidence in the attajnability of our readoust performance goals. 


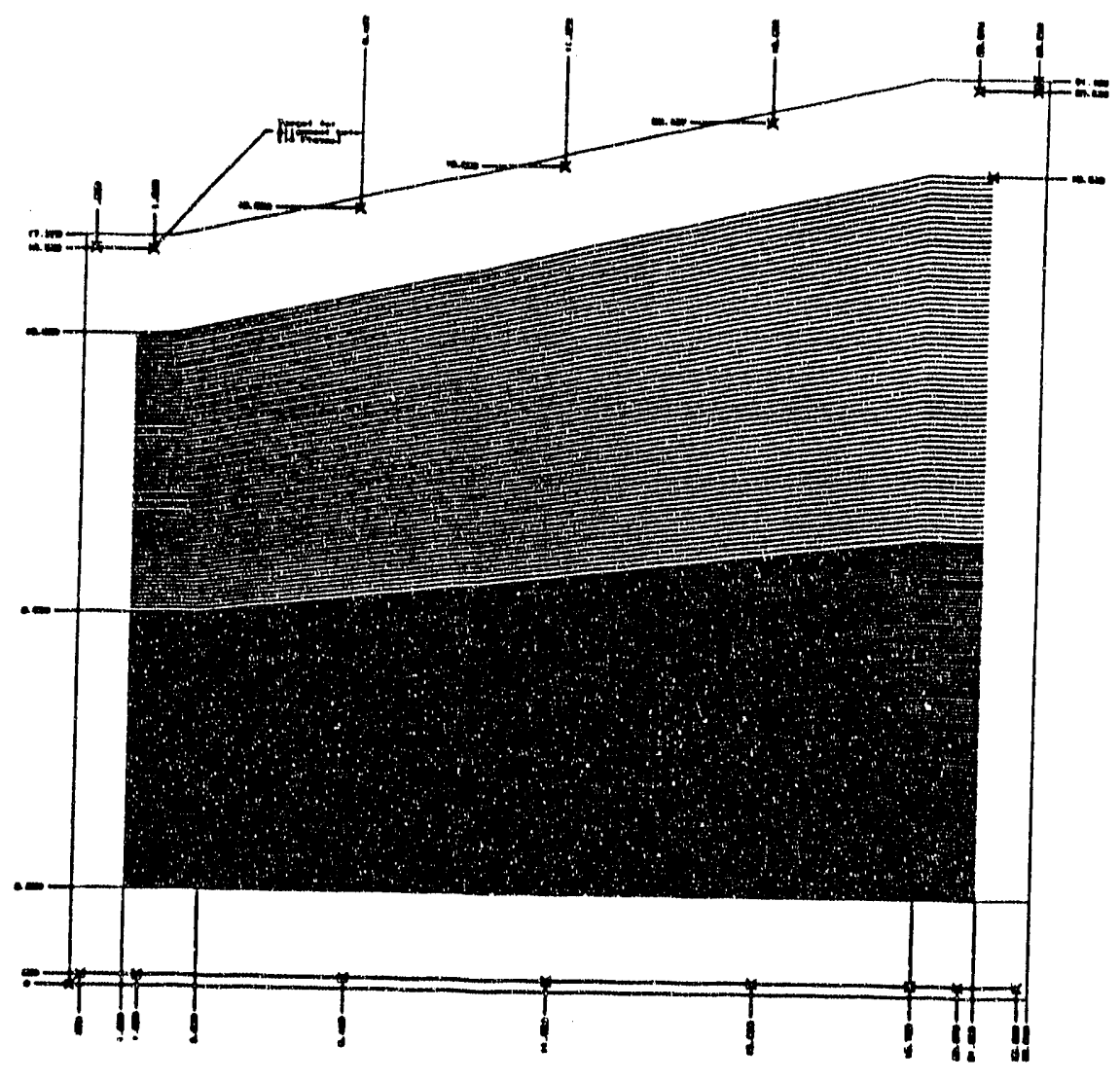

CSC DOUBLET - - CONCEPTUAL DESIGN

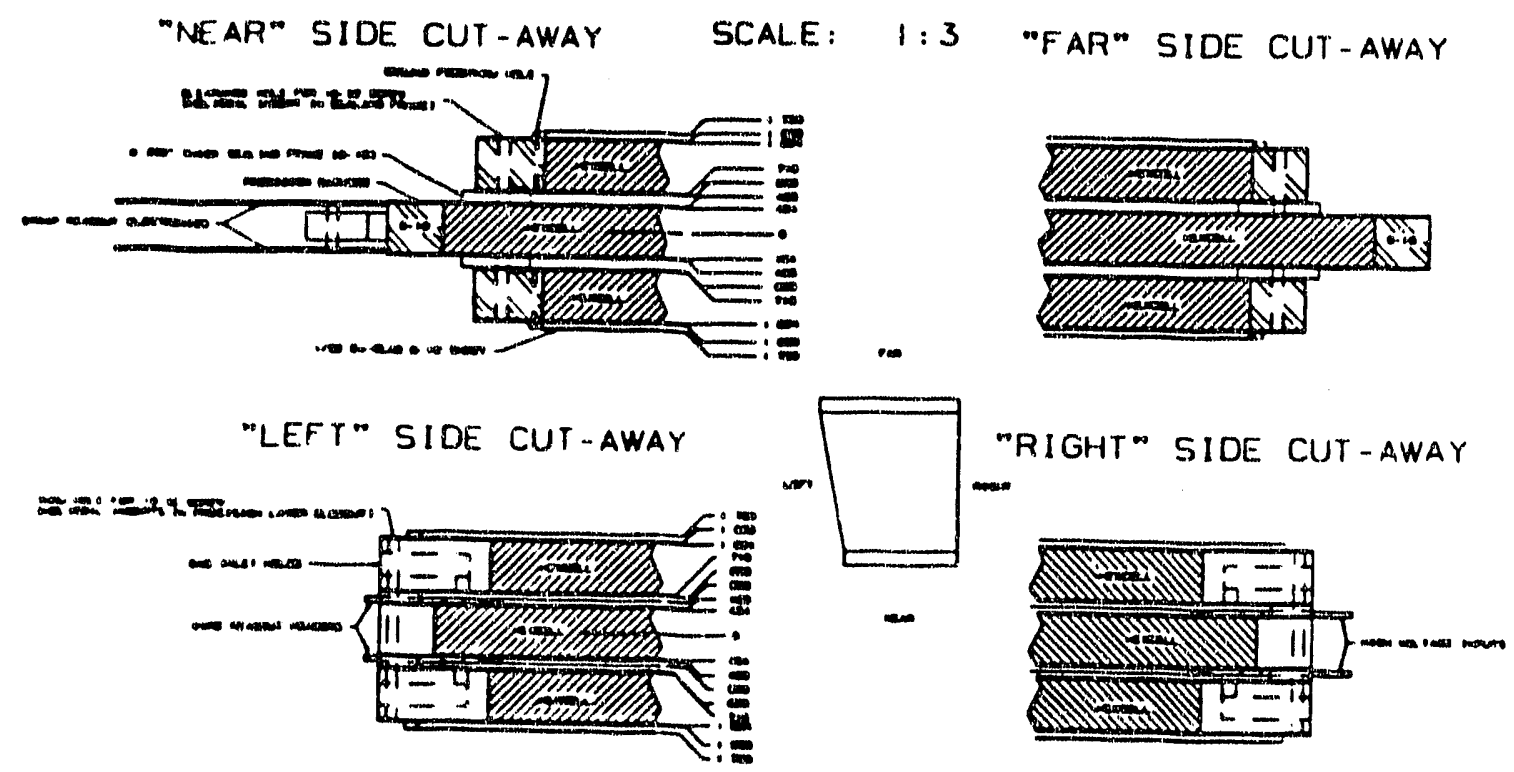

Fig. 3. Prototype Cathode Strip Chamber: plan and cross section views. 
We have studied a smaller chamber which was built with a thin mylar window to allow studies with Fe-55 xrays. Figure 4 shows the position measurements made with Fe55 xrays illuminating the chamber through a collimator that had 0.003 inch slits separated by 0.031 inches. The collimator parallax yields an illuminated region in the chamber of approximately 0.006 inches width. The observed resolution of the peaks corresponds to a position resolution of 150 microns; this is consistent with the collimator illumination and with the position resolution limit due to the range of the $5.95 \mathrm{KeV}$ photoelectron from the Fe-55 xray.

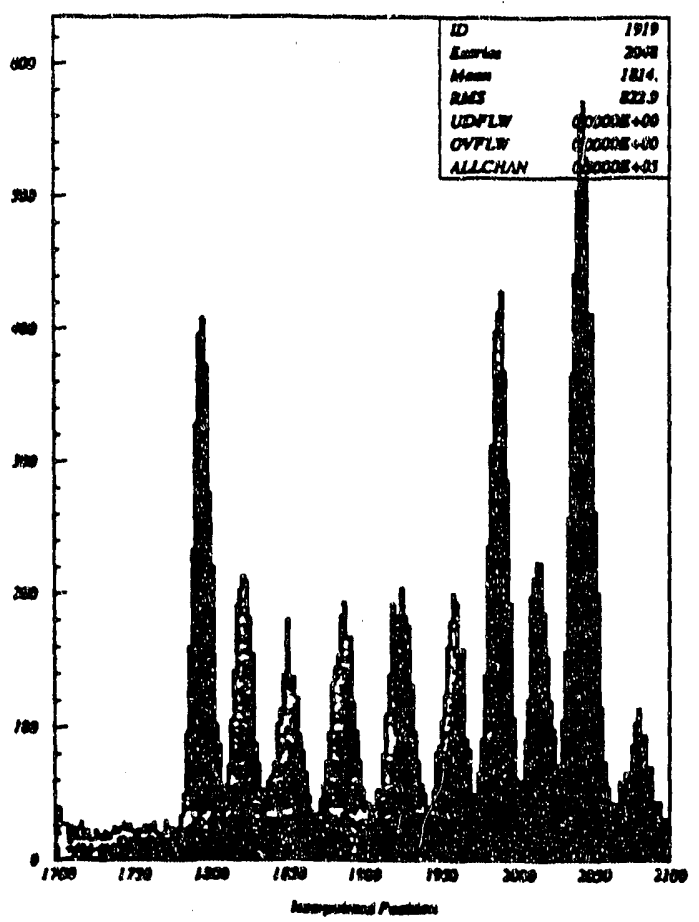

Fig. 4. Distribution of positions of interactions of collimated Fe-55 xrays.

We have used this same chamber to study neutron interactions. The neutron flux in the GEM detector has been calculated (by L. Waters, LANL) to be of the order of $10^{5} \mathrm{~cm}^{-2} \mathrm{sec}^{-1}$. It will be important to understand the neutron interactions in the detec- ' tors in order to develop robust readout and triggering schemes. The studies were done using a Cf-252 source in the laboratory of Prof. L. Osborne at MIT. Various absorbers were interposed between the source and the detector to separate the photon and neutron components. The count rate was found to be due roughly $50 \%$ to photon interactions and $50 \%$ to neutron interactions. We determined the flux of neutrons according to the activity of the source and geometric factors. From this knowledge of the flux and from the measured neutron interaction rate, we calculated a neutron detection efficiency of $0.47 \%$. This 
is some 20 times larger than would be expected due to interactions in the gas. We believe that interactions in the walls of the chambers are dominating the count rate; further tests are planned to verify this interpretation.

\section{Activities of the Coming Year}

Work for the coming year will concentrate on demonstration of the performance of a full-scale CSC, preparation of the GEM technical design report, and development of a full system design with integrated alignment and support features.

The Cathode Strip Chamber prototype is a four-layer module. Each chamber will have 256 strips $.5 \mathrm{~cm}$ wide by $2 \mathrm{~m}$ long, for overall dimensions of the sensitive area 1.28 $\mathrm{m}$ by $2 \mathrm{~m}$. This is well above the average size of the CSC modules envisioned for the final design. Construction of the prototype is well underway by Boston University and Brookhaven National Laboratory personnel. Electronics circuits for readout of the cathode strips (using Amplex chips with preamps) and the anode wires (using hybrid preamps) have been developed and are under test.

This large chamber and smaller prototype chambers of various designs will be extensively tested with cosmic rays, using the Texas Test Rig at the SSCL, and with a muon beam at the RD5 test beam at CERN. We have already installed a gas system and readout and monitoring electronics at CERN to be ready for tests this Fall.

\section{Budget Discussion}

Task L covers SSC detector development. The base operations budget is entered under Task A. Additional operating expenses under Task $\mathrm{L}$ cover the domestic travel required for engineering and design meetings, limited foreign travel for beam tests at CERI, undergraduate student labor, and the engineering salary presently covered under GEM R\&.D. Equipment funds will be allocated to this research within the context of the GEM Experiment Muon Subsystem. The GEM R\&D plan is still under negotiation within the collaboration.

\section{Recent Publications by Task $L$ personnel}

See publications listed under Task A. 


\title{
TASK N: TOPICS IN ELEMENTARY PARTICLE PHYSICS
}

\author{
Faculty: \\ Assistant Professor R. S. Chivukula \\ Graduate Student: \\ B. Balaji
}

\section{Research Accomplishments}

This report describes the research accomplished by Chivukula since the submission of the original OJI proposal in November, 1991. The descriptions that follow are also included in Task $E$ of this proposal.

\section{A. Symmetry Breaking Phenomenology}

If the electroweak symmetry breaking sector contains colored particles weighing a few hundred $\mathrm{GeV}$, then they will be copiously produced at a hadron supercollider. Colored technipions can rescatter into pairs of gauge bosons. As proposed by Bagger, Dawson, and Valencia [1], this leads to gauge boson pair rates far larger than in the standard model. Chirukula, with M. Golden and M. V. Ramana, reconsidered this mechanism in [2], and illustrated it in a model in which the rates can be reliably calculated. They showed that the observation of both an enhanced rate of gauge-boson-pair events and colored particles would be a signal that the colored particles were pseudo-Goldstone bosons of symmetry breaking.

\section{B. Phenomenology of a QCD.like Theory with Many Flavors}

In a recent paper, Cahn and Suzuki argued that a relatively light, $O(500 \mathrm{GeV})$, Higgslike particle exists as a bound state pole in the scattering of technipions [3]. Motivated by this. Chivukula and Golden investigated the occurrence of flavor-singlet scalar resonances in Goldstone boson scattering in the presence of soft explicit symmetry breaking [4]. Using the $O(n)$ model in the large- $n$ limit as an example, they showed that there are two alternatives to the Cahn-Suzuki possibility: First, the "Higgs" can be a "virtual bound state" pole on the second complex-s sheet. In this case, the $\pi_{T} \pi_{T}$ scattering amplitudes may bchave (near threshold) as though there were a Higgs boson present without such a particle actually existing. Second, the particle predicted by Cahn and Suzuki may be unbound, decaying to $\pi_{T} \pi_{T}$. 
Expanding on these ideas, Chivukula and Golden considered the behavior of longitudinal $W$ and $Z$ scattering in the $O(n)$ model [5]. It is generally assumed that elastic $W$ and $Z$ scattering exhibits either resonances below $\sim 1 \mathrm{TeV}$ or enhancements indicating the presence of new strong interactions at or above $1 \mathrm{TeV}$. They showed that there is another possibility: The electroweak symmetry breaking sector may be strongly coupled at low energies because of the presence of large numbers of particles - such as technipions other than the longitudinal components of the $W$ and $Z$. In this case the elastic $W$ and $Z$ scattering amplitudes may be small without the appearance of any discernible resonances, while the total scattering cross sections are large. In such a model, the number of $W W$ and $Z Z$ events never is large: discovering the electroweak symmetry breaking sector depends on the observation of the other particles and the ability to associate them with symmetry breaking. In this sense, the symmetry breaking sector in such a model is "hidden".

Chivukula, Golden, D. Kominis, and Ramana [6] have calculated the production rate of gauge-boson pairs at the SSC in a model with a "hidden" electroweak symmetry breaking sector. They showed that the $W W$ and $Z Z$ scattering signal of electroweak symmetry breaking is swamped by the background. They also calculated the gluon fusion contribution to $Z Z$ production in such a model, and showed that even this contribution is smaller than the background. They conclude, therefore, that we cannot necessarily rely on gauge boson pairs as a signal of the dynamics of symmetry breaking.

With M. Dugan, Chivukula and Golden [7] investigated the dynamics of a QCD-like gauge theory with more than two or three flavors. G neralizing an argument of Sundrum and Soldate (8), they showed that the chiral expansion in a theory with $N$ fiavors breaks down at or below $4 \pi f / \sqrt{N}$. They argued that the breakdown of the chiral expansion is associated with the appearance of physical states other than Goldstone bosons, leading to the surprising conclusion that there must be resonances in a theory with six or eight flavors which are much lighter than one would expect by analogy to QCD.

\section{Higgs Boson Phenomenology}

Chivukula and his student, $V$. Koulorassilopoulos, are investigating the phenomenology of a composite Higgs model at the SSC. When the parameters of the model are adjusted to produce a "Higgs boson" of mass greater than about $600 \mathrm{GeV}$, the effects of the physics responsible for binding the boson start to become significant. They are now estimating the size of these effects. 
Chivukula and Kominis have considered the constraints of triviality on general twoHiggs models. They have shown that, just as in the one-Higgs case, the scalar masses in such a theory are typically constrained to be less than approximately $700 \mathrm{GeV}$. They are now investigating the phenomenology of these acceptable models.

\section{Precision Tests of the Electroweak Interactions}

Golden and Randall recently studied the radiative corrections technicolor theories may make on precisely measured electroweak quantities [9]. Even when technicolor respects the custodial $S U(2)$ symmetry, which guarantees no shift in the low-energy p-parameter, there may be large wave-function renormalizations of the $W$ and $Z$, and these can shift the mass of the $W$ or other parameters. They showed that the corrections to $M_{W}$ induced by the large number of technipions occurring in the one-family TC model may be discernible with higher-statistics measurements that can be made at the Tevatron collider.

In light of the fact ( $c f$. sec. B) that the spectrum of a QCD-like theory with many flavors is likely to be much different than might be expected from QCD, Chivukula, Nugan, and Golden $[10$ ) have re-exarnined estimates of radiative corrections to electroweak parancters in technicolor theories. These corrections are evaluated by one of two techniques: rither one estimates spectral function integrals using scaled QCD data [11] [12], or one uses naive dimensional analysis with a chiral Lagrangian [9] [13]. The former yields corrections (6) clectroweak parameters proportional to the number of flavors and the number of colors, while the latter is proportional to the number of flavors squared and is independent of the number of colors. Chivukula, Dugan and Golden explained this apparent contradiction and argued that, in a theory with more than two or three technifiavors, the technicolor rmonances are likely to be much lighter than naively supposed. If this is the case, the radiative corrections to electroweak parameters due to technicolor may by much larger than navely estimated.

Chivukula, S. B. Selipsky, and E. H. Simmons (Harvard) have probed the possibility of non-oblique electroweak radiative corrections appearing in currently feasible observations [14]. In Exterded Technicolor theories, fermion masses are generated by ETC boson exchange with Technifermions that develop condensates. In addition to effective mass operators, the same ETC bosons exchanged in other channels generate flavor-dependent corrections to couplings to $Z$ and $W$ gauge bosons, with effects proportional to the fermion 
mass. In particular, the top quark mass operator must also (by electroweak symmetry) give a correction to the $\mathrm{Zbb}$ coupling, which turns out to be proportional to $\left(m_{\mathrm{g}} / 3 \mathrm{TeV}\right)$ instead of the Standard Model quadratic dependence. Assuming a minimal theory in which the ETC bosons have no weak charge, the correction is calculable up to a group-dependent factor, and leads to a an $m_{t}$ dependent shift in the $\mathrm{Zbb}$ width of seven times the Standard model value, which should be observable in LEP data currently being obtained.

\section{REFERENCES}

1. J. Bagger, S. Dawson and G. Valencia, Phys. Rev. Lett. 67, 2256 (1991).

2. R. S. Chivukula, M. Goiden and M. V. Ramana, Phys. Rev. Lett. 68, 2883 (1992).

3. R. Cahn and M. Suzuki, Phys. Rev. Lett. 67, 167 (1991).

4. R. S. Chivikula and M. Golden, Nucl. Pbys. B372, 44 (1992).

5. R. S. Chivukula and M. Golden, Phys. Lett. B267, 233 (1991).

G. R. S. Chivukula, D. Kominis, M. Golden, and M. V. Ramana, Boston University preprint BUHEP.92-23 and Phys. Lett. B, in press.

7. R. S. Chivukula, M. Golden, and M. Dugan, Boston University preprint BUHEP-9218.

8. M. Soldate and R. Sundrum Nucl. Phys. B340, 1 (1990).

9. A1. Golden and L. Randall, Nucl. Phys. B361, 3 (1991).

10. R. S. Chivukula, M. Golden, and M. Dugan, Boston University preprint BUHEP.9225.

11. M. Peskin and T. Takeuchi, "Estimation of Oblique Electroweak Corrections," SLAC. PLB.5018 (Nov. 1991).

12. R. N. Cahn and M. Suzuki, Phys. Rev. D 44, 3641 (1991) .

13. A. Longhitano, Phys. Rev. D. 22, 1160 (1980) and Nucl. Phys. B188,118 (1981); R. Renken and M. Peskin, Nucl. Phys. B211,93 (1983); M. Golden and L. Randall, Nucl. Phys. B361, 3 (1990); B. Holdom and J. Terning, Phys. Lett. B247, 88 (1990); A. Dobado. D. Espriu, and M. J. Herrero, Phys. Lett. B255, 405 (1990); H. Georgi, Niucl. Phys. B363, 301 (1991).

14. R. S. Chivukula, E. H. Simmons, and S. B. Selipsky, Phys. Rev. Lett. 89, 575 (1992). 
TASK RS: RESEARCH SUPPORT

Project Director: Professor J. Scott Whitaker

Grant Administrator: Rachel Meisel

\section{Discussion}

The research program described in this proposal is a serious, large-scale effort and requires professional administration. The Research Support Task represents the administrative support that is necessary for the accomplishment of the research goals of this program. The eight projects described earlier in this document encompass the research activities of twelve faculty members, nine research faculty and research associates, and fifteen graduate students. Our administrator, Rachel Meisel, was hired in August 1989 to work full-time on the administration of the projects described in this proposal. The administrator is involved in every aspect of the program. She is the principal liaison with the Boston University purchasing, personnel, and accounting departments.

Meisel is essential to the successful operation of this contract. She has been very effective in using the resources of Boston University in support of this research program. With Meisel shouldering the major burden of contract administration, the scientific productivity of the principal investigators has increased. The proposed budget consists in salary support and fringe benefits for the administrator and limited operating support for adminsitrative functions. 

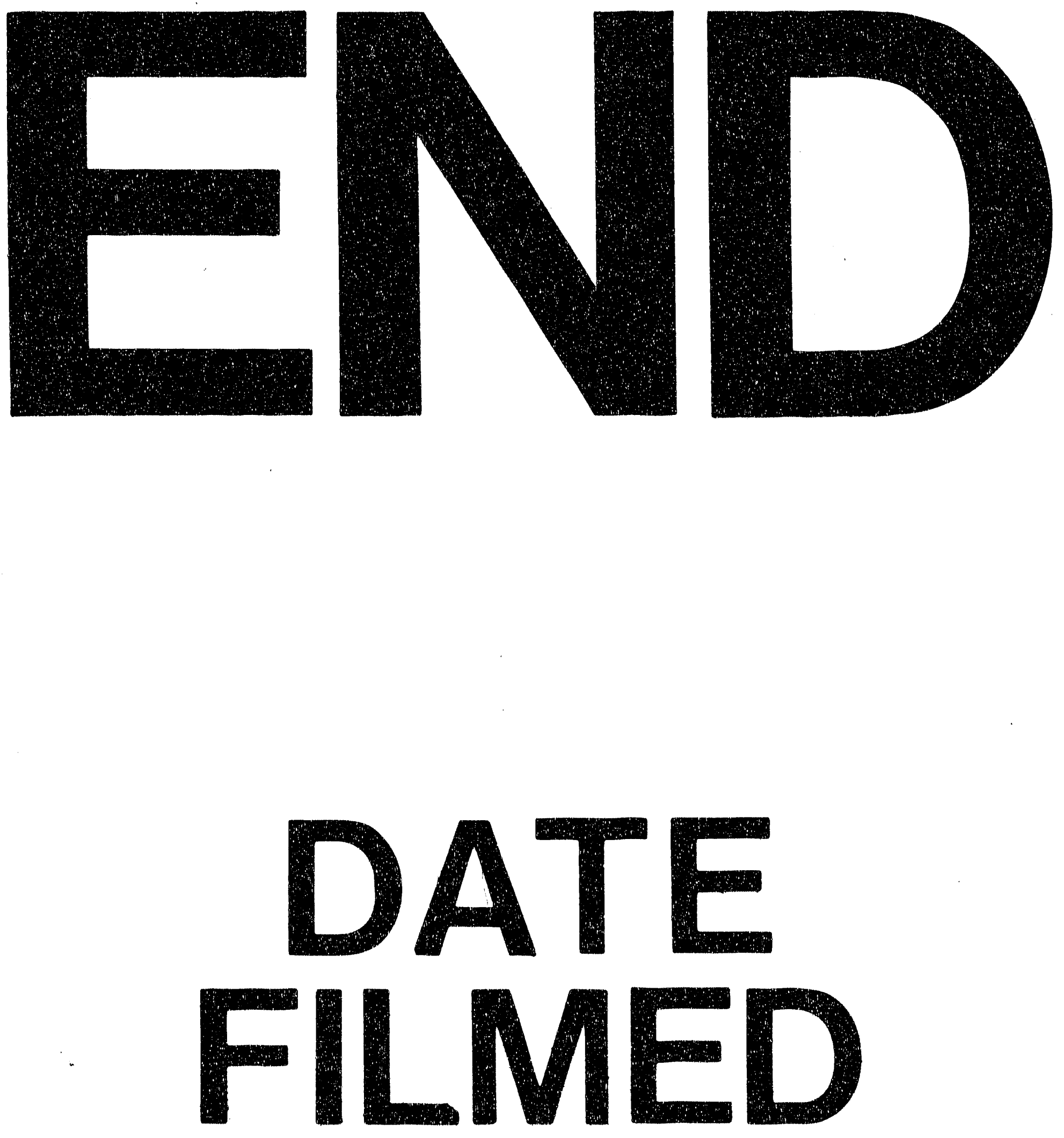

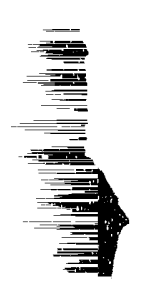

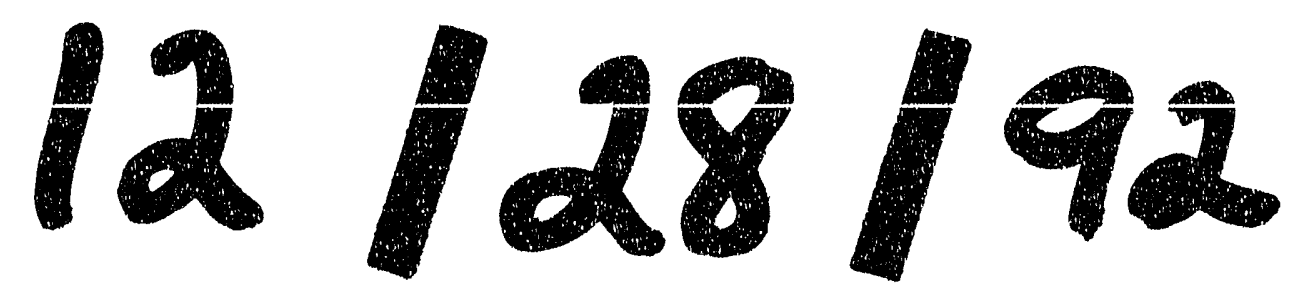


$\underline{\underline{ }}$ 